\title{
HOMFLY polynomials, stable pairs and motivic Donaldson-Thomas invariants
}

\author{
Duiliu-Emanuel Diaconescu, Zheng Hua and Yan Soibelman
}

Hilbert scheme topological invariants of plane curve singularities are identified to framed threefold stable pair invariants. As a result, the conjecture of Oblomkov and Shende on HOMFLY polynomials of links of plane curve singularities is given a Calabi-Yau threefold interpretation. The motivic Donaldson-Thomas theory developed by $\mathrm{M}$. Kontsevich and the third author then yields natural motivic invariants for algebraic knots. This construction is motivated by previous work of V. Shende, C. Vafa and the first author on the large $N$-duality derivation of the above conjecture.

1. Introduction

1.1. The conjectures of Oblomkov et al.

\subsubsection{A conjecture for colored HOMFLY polynomials}


518 Duiliu-Emanuel Diaconescu, Zheng Hua and Yan Soibelman

2.2. A $C$-framed subcategory

2.3. Properties of $C$-framed limit slope stable objects

3. Stable pairs at small $b$

3.1. A stability criterion

3.2. Moduli spaces of decorated sheaves

3.3. Relation to nested Hilbert schemes

3.4. Relation to small $b$ moduli spaces

4. Motivic invariants at small $b$

4.1. Review of motivic DTs invariants

4.2. Motivic weights at small $b$

4.3. Local toric models

4.4. Motivic weights in local model

4.5. Comparison with refined Hilbert scheme invariants

Appendix A. Wall-crossing formula

A.1. Critical stability parameters $\quad 585$

A.2. Motivic Hall algebra identities $\quad 590$

$\begin{array}{ll}\text { A.3. Summing over critical values } & 593\end{array}$

References

$\mathbf{5 9 7}$

\section{Introduction}

The starting point of this work is a conjecture of Oblomkov and Shende [42] relating the HOMFLY polynomial of the link of a plane curve singularity to 
topological invariants of its Hilbert scheme of points. It was then explained in [11] that this conjecture has a natural physical interpretation in terms of large $N$ duality for conifold transitions. The conifold transition is a topology changing process from a smooth hypersurface

$$
x z-y w=\mu, \quad \mu \neq 0,
$$

in $\mathbb{C}^{4}$ to a small resolution the conifold singularity

$$
x z-y w=0,
$$

which is isomorphic to the total space $Y$ of the rank two bundle $\mathcal{O}_{\mathbb{P}^{1}}(-1)^{\oplus 2}$ on $\mathbb{P}^{1}$. In this context, the construction of [11] assigns to an algebraic knot $K$ in $S^{3}$ a Lagrangian cycle $M_{K}$ in $Y$ which intersects a singular plane curve $C^{\circ}$ contained in a fiber of $Y \rightarrow \mathbb{P}^{1}$ along a circle. Moreover, $C^{\circ}$ has a unique singular point at the intersection with the zero section, its link being isotopic to $K$. Then large $N$ duality leads to a conjectural relation between HOMFLY polynomials of algebraic knots and Gromov-Witten theory on $Y$ with Lagrangian boundary conditions on $M_{K}$. This conjecture has been tested in [11] by explicit $\mathbf{A}$-model computations for torus knots.

The relation between large $N$ duality and the conjecture of Oblomkov and Shende follows from the observation that Gromov-Witten theory is conjecturally equivalent to Donaldson-Thomas theory [32], and also stable pair theory [45]. For Gromov-Witten theory counting stable maps with compact domain without boundary, these relations have been proven for toric threefolds in [33,34]. String duality arguments [28,31,44] predict that GromovWitten theory with Lagrangian boundary conditions should be similarly related to certain certain D6-D2-D0 counting invariants. The latter have not been given a rigorous mathematical construction since a definition of Donaldson-Thomas (DT) or stable pair theory with Lagrangian boundary conditions is not known so far. In certain special cases, such as Lagrangian cycles associated to the unknot, one can employ relative DT or stable pair theory in order to fill this gap. Then the correspondence reduces via [25] to certain identities for cubic Hodge integrals on the moduli space of curves, which have been proven in $[29,30,43]$. This approach is not however expected to work for more general Lagrangian cycles, in particular for the Lagrangian cycles for algebraic knots constructed in [11]. Therefore, one is left with the question whether there is a DT/stable pair construction for counting invariants corresponding via large $N$ duality to general algebraic knots.

The main claim of the present paper is that there is a natural construction of such invariants in terms of stable pairs subject to a framing condition 
explained below. Given a singular plane curve $C^{\circ}$ in a fiber of the projection $Y \rightarrow \mathbb{P}^{1}$, there is a natural moduli space of $C^{\circ}$-framed stable pairs on $Y$. These are pairs $\mathcal{O}_{Y} \stackrel{s}{\longrightarrow} F$ on $Y$ where $F$ is topologically supported on the union of $C^{\circ}$ with the zero section $C_{0} \subset Y$, and has multiplicity one along $C^{\circ}$. Then the main result is that such moduli spaces are related to the nested Hilbert schemes employed in $[41,42]$ by a variation of stability condition. For technical reasons, this is proven embedding of the affine curve $C^{\circ}$ in a suitable compact Calabi-Yau threefold $X$. In particular, the embedding will factor through the natural projective completion $C \subset \mathbb{P}^{2}$ of $C^{\circ}$. Using previous results on stability conditions for perverse coherent sheaves [50,51], the nested Hilbert schemes of $[41,42]$ are then geometrically related to moduli spaces of framed stable objects in a certain stability chamber.

Enumerative invariants for $C$-framed stable pairs are defined by integration of a certain constructible function $\nu$ on the moduli space of $C^{\circ}$-framed stable pairs. Since the Hilbert scheme invariants used in [42] are topological, one can simply take $\nu=1$ obtaining the topological Euler numbers of the moduli spaces. Then a wall-crossing formula shows that the resulting invariants are then in agreement with those of [42]. Alternative constructions may be carried out, using either Behrend constructible functions [2] as in [24] or motivic weight functions as in [26]. Motivated by previous connections between motivic and refined DT invariants $[3,12,37,38]$, the second approach will be considered in this paper. Assuming the foundational aspects of [26], it will be shown that the virtual motivic invariants of $C$-framed objects are in agreement with the refined conjecture formulated in [41] if certain technical conditions are met. Removing the technical conditions in question reduces to a comparison conjecture between motivic weights of stable pairs and sheaves (see Section 4.2) which is at the moment open.

Appearance of motivic DT invariants supports an old idea of S. Gukov and third author that there should exist a motivic knot invariants theory. In such theory skein relations should correspond to wall-crossing formulas for the motivic DT invariants introduced in [26] (and further developed in [27]). Knot invariants themselves should be derived from an appropriate three-dimensional (3D) Calabi-Yau category.

The idea can be traced back to [16], where Khovanov-Rozansky theory was linked to the count of BPS states in topological string theory. It was further developed in [13] in the form of a conjecture about knot superpolynomial. After the work [26] of Kontsevich and third author it became clear that motivic DT-invariants introduced in the loc. cit. provide the right mathematical foundation for the notion of (refined) BPS state. This was pointed out in [12] based on physics arguments, rigorous mathematical statements 
confirming this claim being first formulated and proved in [3]. Further results along these lines have been obtained in $[37,38]$. The parameter $y$ that appears in knot invariants should correspond to the motive $\mathbb{L}=\left[\mathbb{A}^{1}\right]$ of affine line in the theory of motivic DT-invariants. Then the question is: what is an appropriate 3-demensional Calabi-Yau category? From the point of view of the large $N$ duality it is natural to expect that the 3D Calabi-Yau category should be somehow derived from the resolved conifold $Y$. Unfortunately it is difficult to make this idea mathematically precise since $Y$ is noncompact (as well as the Lagrangian cycle $M_{K}$ ). One can see that the partition function for the unknot derived in [44] coincides with the motivic DT-series for the 3D Calabi-Yau category generated by one spherical object (both are given essentially by the quantum dilogarithm). But there was no general conjecture about the desired relationship. Although such a conjecture does not exist at present, the works $[41,42]$ give a hope that it can be formulated soon. Our paper can be considered as another step in this direction.

A more detailed overview including technical details is presented at length below.

\subsection{The conjectures of Oblomkov et al.}

Let $C^{\circ} \subset \mathbb{C}^{2}$ be a reduced pure dimension one curve with one singular point $p \in C^{\circ}$. Let $H_{p}^{n}\left(C^{\circ}\right)$ be the punctual Hilbert scheme parameterizing length $n$ zero-dimensional subschemes of $C^{\circ}$ with topological support at $p$. Let $m: H_{p}^{n}\left(C^{\circ}\right) \rightarrow \mathbb{Z}$ be the constructible function assigning to any subscheme $Z \subset C^{\circ}$ with topological support at $p$ the minimal number of generators of the defining ideal $\mathcal{I}_{Z, p} \subset \mathcal{O}_{C^{\circ}, p}$ at $p$. For any scheme, $X$ of finite type over $\mathbb{C}$, and any constructible function $\nu: X \rightarrow \mathbb{Z}$ let

$$
\int_{X} \nu d \chi=\sum_{n \in \mathbb{Z}} n \chi\left(\nu^{-1}(n)\right)
$$

where $\chi$ denotes the topological Euler character. Then let

$$
Z_{C^{\circ}, p}(q, a)=\sum_{n \geq 0} q^{2 n} \int_{H_{p}^{n}\left(C^{\circ}\right)}\left(1-a^{2}\right)^{m} d \chi .
$$

Let $K_{C^{\circ}, p}$ denote the link of the plane curve singularity at $p$. Let $P_{K_{C^{\circ}, p}}(a, q)$ denote the HOMFLY polynomial of $K_{C^{\circ}, p}$. It satisfies the skein relation of the type:

$$
a P_{L_{+}}-a^{-1} P_{L_{-}}=\left(q-q^{-1}\right) P_{L_{0}}
$$


As opposed to [42], the HOMFLY polynomial will be normalized such that it takes value

$$
\frac{a-a^{-1}}{q-q^{-1}}
$$

for the unknot. Then the conjecture of Oblomkov and Shende [42] states that

$$
P_{K_{C^{\circ}, p}}(q, a)=(a / q)^{\mu-1} Z_{C^{\circ}, p}(q, a)
$$

where $\mu$ is the Milnor number of the singularity at $p$.

1.1.1. Refinement The correspondence between knot polynomial invariants and Hilbert scheme invariants of curve singularities admits a refined generalization due to Oblomkov et al [41]. Given an algebraic knot or link $K$, let $P_{K}^{\mathrm{ref}}(q, a, y)$ denote the refined HOMFLY polynomial introduced in $[13$, 16]. This is the polynomial invariant called reduced superpolynomial in [13], which specializes to the HOMFLY polynomial at $y=-1$. In the previous notation consider the incidence cycle

$$
H_{p}^{[l, r]}\left(C^{\circ}\right) \subset H_{p}^{l}\left(C^{\circ}\right) \times H_{p}^{l+r}\left(C^{\circ}\right)
$$

parameterizing pairs of ideals $(J, I)$ in the local structure ring $\mathcal{O}_{C^{\circ}, p}$ satisfying the following condition:

$$
m_{p} J \subseteq I \subseteq J
$$

where $m_{p} \subset \mathcal{O}_{C^{\circ}, p}$ is the maximal ideal of the singular point. Let $H_{p}^{[l, r]}\left(C^{\circ}\right)$ be equipped with the reduced induced subscheme structure and

$$
Z_{C^{\circ}, p}^{\mathrm{ref}}(q, a, y)=\sum_{l, r \geq 0} q^{2 l} a^{2 r} y^{r^{2}} P_{y}\left(H_{p}^{[l, r]}\left(C^{\circ}\right)\right),
$$

where $P_{y}$ denotes the virtual Poincaré polynomial (also known as Serre polynomial). Then Oblomkov et al. [41] conjecture the following relation:

$$
P_{K}^{\mathrm{ref}}(q, a, y)=\left(\frac{a}{q}\right)^{\mu-1} Z_{C^{\circ}, p}^{\mathrm{ref}}(q, a, y) .
$$

\subsection{Framed stable pair invariants of the conifold}

The resolved conifold $Y$ is a small crepant resolution of the nodal hypersurface $x z-y w=0$ in $\mathbb{C}^{4}$. It can be easily identified with the total space 
of the rank two bundle $\operatorname{Tot}\left(\mathcal{O}_{\mathbb{P}^{1}}(-1) \oplus \mathcal{O}_{\mathbb{P}^{1}}(-1)\right)$ such that the exceptional cycle $C_{0} \simeq \mathbb{P}^{1}$ of the resolution is the zero Section.

There is closed embedding $C^{\circ} \hookrightarrow Y$ which factors through the natural embedding of $C$ in a fiber of the projection $Y \rightarrow \mathbb{P}^{1}$. Therefore, the curve $C^{\circ}$ in the conjecture of Oblomkov and Shende is naturally identified with a vertical complete intersection on $Y$. Recall [45] that a stable pair on $Y$ is determined by the data $(F, s)$, where $F$ is a pure dimension one coherent torsion sheaf on $Y$, and $s: \mathcal{O}_{Y} \rightarrow F$ is a morphism with zero-dimensional cokernel. Note that $F$ will not be assumed to have proper support. Let $\mathcal{I}_{C^{\circ}}$ be the defining ideal sheaf of $C^{\circ} \subset Y$. A $C^{\circ}$-framed stable pair on $Y$ is a stable pair $(F, s)$ such that

- $F$ is topologically supported on the union $C^{\circ} \cup C_{0}$;

- The annihilator ideal $A n n(F)$ of $F$ is a subsheaf of the defining ideal $\mathcal{I}_{C}$ of $C^{\circ}$ and the quotient $\mathcal{I}_{C^{\circ}} / \operatorname{Ann}(F)$ is topologically supported on the zero Section $C_{0}$.

Note that the second condition is equivalent to the requirement that the scheme theoretic support $Z_{F}$ of $F$ have at most two irreducible components, $C^{\circ}$ and an additional component supported on $C_{0}$, which may be empty. The numerical invariants of a $C^{\circ}$-framed stable pair on $Y$ will be the generic multiplicity $r$ of $F$ along the zero Section, and $l=\chi(\operatorname{Coker}(s))$.

Let $\bar{Y}=\mathbb{P}\left(\mathcal{O}_{Y}(-1)^{\oplus 2} \oplus \mathcal{O}_{Y}\right)$ be a projective completion of $Y$, and $C \subset$ $\bar{Y}$ the resulting projective completion of $C^{\circ}$. Projective plane curve $C$ is contained in a fiber of the projection $\bar{Y} \rightarrow \mathbb{P}^{1}$. According to [45], there exists a fine projective moduli space $\mathcal{P}(\bar{Y}, r, n)$ of stable pairs $(G, v)$ on $\bar{Y}$, where $\operatorname{ch}_{2}(G)=[C]+r\left[C_{0}\right]$, and $\chi(G)=n$. Then it can be easily proved that there exists a fine quasi-projective moduli space $\mathcal{P}\left(Y, C^{\circ}, r, l\right)$ of $C^{\circ}$-framed stable pairs on $Y$ with $l=n-\chi\left(\mathcal{O}_{C}\right)$. Moreover, $\mathcal{P}\left(Y, C^{\circ}, r, l\right)$ is the locally closed subscheme of $\mathcal{P}(\bar{Y}, r, n)$ determined by the conditions:

- $\operatorname{Ann}(G) \subset \mathcal{I}_{C}$;

- The support of $\operatorname{Coker}(v)$ is contained in the open part $Y \subset \bar{Y}$.

Let $\mathcal{P}^{\circ}(\bar{Y}, r, n)$ denote the open subspace of $\mathcal{P}(\bar{Y}, r, n)$ parameterizing pairs satisfying only the above second condition.

Counting invariants $P^{\nu}\left(Y, C^{\circ}, r, n\right)$ are defined by integrating a constructible function $\nu$ on the ambient moduli space $\mathcal{P}^{\circ}(\bar{Y}, r, n)$ over the subspace $\mathcal{P}\left(Y, C^{\circ}, r, n\right)$. Several choices are in principle available for such a constructible function: the constant function $\nu=1$, Behrend's constructible 
function [2], or the motivic weight function conjectured in [26]. Two cases will be considered in this paper, namely $\nu=1$, or the motivic weight function of $[26]$.

In the first case, the resulting invariants are simply topological Euler numbers of moduli spaces,

$$
P^{\text {top }}\left(Y, C^{\circ}, r, n\right)=\chi\left(\mathcal{P}\left(Y, C^{\circ}, r, n\right)\right)
$$

In the second case, assuming the foundational problems solved, the construction of [26] produces a motivic weight function $\nu^{\text {mot }}$ together with a finite stratification of $\left\{\mathcal{S}_{\alpha}\right\}$ of the moduli space $\mathcal{P}^{\circ}(\bar{Y}, r, n)$ such that $\nu^{\text {mot }}$ takes a constant value $\nu_{\alpha}^{\text {mot }}$ on each locally closed stratum $\mathcal{S}_{\alpha}$. The motives $\nu_{\alpha}^{\text {mot }}$ belong to a certain ring of motives presented in detail in [26, Sect. 4.3 and 6.2] which contains the Grothendieck ring $K_{0}(\operatorname{Var} / \mathbb{C})$ of complex algebraic varieties as a subring, as well as a formal square root $\mathbb{L}^{1 / 2}$ and formal inverses $\mathbb{L}^{-1},[G L(k, \mathbb{C})]^{-1}, k \in \mathbb{Z}_{\geq 1}$. Then the motivic DT invariants of $C^{\circ}$-framed stable pairs are defined by

$$
P^{\operatorname{mot}}\left(Y, C^{\circ}, r, n\right)=\sum_{\alpha}\left[\mathcal{S}_{\alpha}\right] \nu_{\alpha}^{\text {mot }}
$$

where $\left[\mathcal{S}_{\alpha}\right] \in K_{0}(\operatorname{Var} / \mathbb{C})$ is the Chow motive of the stratum $\mathcal{S}_{\alpha}$. In both cases, let

$$
Z^{\nu}\left(Y, C^{\circ}, u, T\right)=\sum_{n \in \mathbb{Z}_{\geq 0}} \sum_{r \geq 0} P^{\nu}\left(Y, C^{\circ}, r, n\right) u^{n} T^{r}
$$

be the resulting generating function. Let also $P^{\nu}(Y, r, n)$ denote the corresponding counting invariants for stable pairs $(F, s)$ on $Y$ with $\operatorname{ch}_{2}(Y)=r\left[C_{0}\right]$ and $n=\chi(F)$, and

$$
Z^{\nu}(Y, u, T):=\sum_{n \in \mathbb{Z}} \sum_{r \geq 0} P^{\nu}(Y, r, n) u^{n} T^{r}
$$

their generating function. Then large $\mathrm{N}$ duality [11] predicts a conjectural factorization formula

$$
Z^{\nu}\left(Y, C^{\circ}, u, T\right)=Z^{\nu}(Y, u, T) Z^{\nu}\left(C^{\circ}, u, T\right)
$$

where $Z^{\nu}\left(C^{\circ}, u, T\right)$ is a formal power series in $(T, u)$, possibly up to multiplication by an overall Laurent monomial in $(T, u)$. This is a reflection of the natural factorization of Wilson loop expectation values in large $N$ 
Chern-Simons theory,

$$
\left\langle W_{K}(U)\right\rangle_{\mathrm{CS}, N \rightarrow \infty}=P_{K}(q, a) Z_{\mathrm{CS}}(q, a),
$$

where $Z_{\mathrm{CS}}(q, a)$ is the large $N$ limit of the $U(N)$ Chern-Simons partition function on $S^{3}$. Furthermore $Z^{\nu}\left(C^{\circ}, u, T\right)$ is conjectured to have an intrinsic interpretation in terms of D6-D2-D0-bound state counting on $Y$ and is conjecturally related to the generating function $Z_{C^{\circ}, p}(q, a)$ in Equation (1.2) or its refined counterpart (1.3) as explained in detail below. Let

$$
Z_{C^{\circ}}^{\text {top }}(q, a)=\sum_{n \geq 0} q^{2 n} \int_{H^{n}\left(C^{\circ}\right)}\left(1-a^{2}\right)^{m} d \chi
$$

be the global version of Equation (1.2), where the punctual Hilbert scheme $H_{p}^{n}\left(C^{\circ}\right)$ is replaced by the Hilbert scheme $H^{n}\left(C^{\circ}\right)$ of length $n$ zerodimensional subschemes of $C^{\circ}$ with no support condition. Similarly, consider the following global motivic version of (1.3):

$$
Z_{C^{\circ}}^{\mathrm{mot}}(a, q)=\sum_{l, r \geq 0} q^{2 l} a^{2 r} \mathbb{L}^{r^{2} / 2}\left[H^{n}\left(C^{\circ}\right)\right]
$$

where $\left[H^{n}\left(C^{\circ}\right)\right] \in K_{0}(\operatorname{Var} / \mathbb{C})$ denotes the Chow motive of the Hilbert scheme. Taking the virtual Poincaré polynomial with compact support, one obtains the global refined generating function:

$$
Z_{C^{\circ}}^{\mathrm{ref}}(a, q, y)=\sum_{l, r \geq 0} q^{2 l} a^{2 r} y^{r^{2}} P_{y}\left(H^{n}\left(C^{\circ}\right)\right) .
$$

Note that a simple stratification argument shows that

$$
Z_{C^{\circ}}^{\mathrm{top}}(a, q)=\left(1-q^{2}\right)^{1-\chi\left(C^{\circ}\right)} Z_{C^{\circ}, p}(a, q)
$$

respectively

$$
Z_{C^{\circ}}^{\mathrm{ref}}(a, q, y)=\left(\sum_{n \geq 0} q^{2 n} P_{y}\left(S^{n}\left(C^{\circ} \backslash\{p\}\right)\right)\right) Z_{C^{\circ}, p}^{\mathrm{ref}}(a, q, y),
$$

where $S^{n}\left(C^{\circ} \backslash\{p\}\right)$ are the symmetric powers of the punctured curve $C^{\circ} \backslash$ $\{p\}$. The compactly supported cohomology $H_{c}^{k}\left(C^{\circ} \backslash\{p\}\right), k \geq 0$ is endowed with Deligne's weight filtration. Let $h_{c}^{k, w}\left(\left(C^{\circ} \backslash\{p\}\right)\right.$ be the dimension of the 
successive quotient of weight $w$. Then, using the results of [5], the above formula can be rewritten in closed form as follows:

$$
Z_{C^{\circ}}^{\mathrm{ref}}(a, q, y)=\prod_{k, w \geq 0}\left(\frac{1}{1-(-1)^{w} y^{k} q^{2}}\right)^{(-1)^{w} h_{c}^{k, w}\left(C^{\circ} \backslash\{p\}\right)} Z_{C^{\circ}, p}^{\mathrm{ref}}(a, q, y) .
$$

Then large $N$ duality leads to the conjecture that there is a monomial change of variables $T=T\left(a, q, \mathbb{L}^{1 / 2}\right), u=u\left(a, q, \mathbb{L}^{1 / 2}\right)$ such that the following identity holds:

$$
Z^{\mathrm{mot}}\left(Y, C^{\circ}, T, u\right)=a^{\alpha} q^{\beta} \mathbb{L}^{\gamma} Z_{C^{\circ}}^{\operatorname{mot}}(q, a)
$$

for some $\alpha, \beta \in \mathbb{Z}, \gamma \in \frac{1}{2} \mathbb{Z}$. Taking virtual Poincaré polynomials with compact support yields a similar identity for refined invariants

$$
Z^{\mathrm{ref}}\left(Y, C^{\circ}, T, u, y\right)=a^{\alpha} q^{\beta} y^{2 \gamma} Z_{C^{\circ}}^{\mathrm{ref}}(q, a, y),
$$

subject again to a monomial change of variables $T=T(a, q, y), u=u(a, q, y)$. Specializing the refined identity to $y=1$ yields a similar conjectural relation

$$
Z^{\text {top }}\left(Y, C^{\circ}, T, u\right)=a^{\alpha} q^{\beta} Z_{C^{\circ}}^{\text {top }}(q, a) .
$$

for topological invariants invariants.

The main result of this paper, Theorem 1.1 below, proves an identity of the form (1.9) for framed stable pair invariants on a smooth projective Calabi-Yau threefold $X$. Compactness is needed here for technical reasons, as the proof relies heavily on the wall-crossing formalism of $[24,26]$ applied to abelian categories of perverse coherent sheaves as in [50,51]. As explained in Section 1.4, the threefold $X$ is a smooth crepant resolution of a nodal threefold $X_{0}$ and contains a projective completion $C \subset \mathbb{P}^{2}$ of $C^{\circ}$, assumed to be smooth away from $p$. Moreover, a compact version of the motivic identity (1.8) can be in principle derived along the same lines from the formalism of [26], assuming the required foundational results as well as certain technical results on motivic weights. The main steps are summarized in Section 1.6 and explained in detail in Section 4.

\subsection{Embedding in a compact Calabi-Yau threefold}

Theory of stable pairs of Pandharipande and Thomas deals with compact varieties. Since the resolved conifold $Y$ is noncompact we need to formulate the problem in an appropriate compactification. We start with some 
generalities. Let $X_{0}$ be a projective Calabi-Yau threefold with a single conifold singularity $q \in X_{0}$. Since all ordinary double points are analytically equivalent, the formal neighborhood of $q \in X_{0}$ is isomorphic to the formal neighborhood of the origin in the singular hypersurface $x z-y w=0$ in $\mathbb{C}^{4}$. Suppose moreover, there exists a Weil divisor $\Delta \simeq \mathbb{P}^{2} \subset X_{0}$ containing $q$ which is locally determined by $z=0$. Blowing up $X_{0}$ along the divisor $\Delta$ yields a crepant resolution $X \rightarrow X_{0}$, the exceptional locus being a $(-1,-1)$ curve $C_{0} \subset X$. Let $D$ be the strict transform of $\Delta$ in $X$. A local computation shows that $D \simeq \Delta$ intersects $C_{0}$ transversely at a point $p$.

Although the considerations below are not particular to a specific model, an example will be provided next for concreteness. Let $X^{-}$be a smooth elliptic fibration with a section over the Hirzebruch surface $\mathbb{F}_{1}$. Let $D^{-} \subset$ $X^{-}$denote the image of the canonical section, and $C_{0}^{-} \subset D^{-}$the unique $(-1)$ curve on $D^{-}$. As shown in [39] using toric methods, there exists a morphism $X^{-} \rightarrow X_{0}$ contracting the curve $C_{0}^{-}$, where $X_{0}$ is a nodal CalabiYau threefold. Moreover there is a second smooth crepant resolution of $X \rightarrow$ $X_{0}$ equipped with a projection to $\mathbb{P}^{2}$, and a section $D \simeq \mathbb{P}^{2}$. The exceptional locus is in this case a rational $(-1,-1)$ curve intersecting $D$ transversely at a point $p$. More examples with two or four conifold singularities where $D$ is a toric surface have been studied in the context of large $N$ duality in [10].

In this context, let $\Gamma \subset X_{0}$ be a reduced irreducible plane curve contained in the Weil divisor $\Delta \simeq \mathbb{P}^{2}$ passing through the conifold point $q$. Suppose $\Gamma$ has a singularity at $q$ and is otherwise smooth. Let $C \subset X$ be the strict transform of $\Gamma$ in $X$. Note that $C$ is a plane curve in $D \simeq \mathbb{P}^{2} \subset X$, and the restriction of the contraction $X \rightarrow X_{0}$ to $C$ is an isomorphism $C \stackrel{\sim}{\longrightarrow} \Gamma$. Moreover $C$ intersects the exceptional curve $C_{0} \subset X$ at the point $p$, which is the only singular point of $C$ under the current assumptions.

By analogy with Section 1.2 a stable pair $(F, s)$ on $X$ will be called $C$-framed of type $(r, n) \in \mathbb{Z}_{\geq 0} \times \mathbb{Z}$

- $F$ is topologically supported on the union $C \cup C_{0}$;

- $\operatorname{ch}_{2}(F)=[C]+r\left[C_{0}\right], \chi(F)=n$.

Then there is a closed subscheme $\mathcal{P}(X, C, r, n) \subset \mathcal{P}(X, \beta, n)$, with $\beta=[C]+$ $r\left[C_{0}\right]$ parameterizing $C$-framed stable pairs.

Enumerative invariants are defined as explained above Equation (1.5) by integration with respect to an appropriate constructible function $\nu$ on the ambient space $\mathcal{P}(X, \beta, n)$. For $\nu=1$, the resulting invariants are topological Euler numbers of the moduli spaces $\mathcal{P}(X, C, r, n)$ and they will be denoted by $P^{\text {top }}(X, C, r, n)$. Taking $\nu$ to be the motivic weight function $[26$, Section 
6.2] on the ambient space $\mathcal{P}(X, \beta, n)$, one obtains motivic $C$-framed stable pair invariants $P^{\operatorname{mot}}(X, C, r, n)$. Their generating functions are

$$
Z^{\nu}(X, C, T, u)=\sum_{n \in \mathbb{Z}} \sum_{r \geq 0} T^{r} u^{n} P^{\nu}(X, C, r, n) .
$$

One similarly defines constructible function invariants $P^{\nu}\left(X, C_{0}, r, n\right)$ for stable pairs $(F, s)$, where $F$ is topologically supported on $C_{0}$, and has numerical invariants $\operatorname{ch}_{2}(F)=n\left[C_{0}\right], \chi(F)=n$. Their generating function will be denoted by $Z^{\nu}\left(X, C_{0}, T, u\right)$. In order to make a connection with the large $N$ duality conjectures in Section 1.2 , note that

$$
Z^{\nu}(Y, T, u)=Z^{\nu}\left(X, C_{0}, T, u\right)
$$

since the formal neighborhood of $C_{0}$ in $X$ is isomorphic to the formal neighborhood of the zero section in $Y$.

As anticipated in Section 1.2, the generating functions (1.6), (1.7) admit natural compact versions

$$
Z_{C}^{\mathrm{top}}(q, a)=\sum_{n \geq 0} q^{2 n} \int_{H^{n}(C)}\left(1-a^{2}\right)^{m} d \chi
$$

respectively

$$
Z_{C}^{\mathrm{mot}}(q, a)=\sum_{n \geq 0} q^{2 n} a^{2 r} \mathbb{L}^{r^{2} / 2}\left[H^{[n, r]}(C)\right]
$$

The notation is analogous to Section 1.1, except that the punctured curve $C^{\circ}$ is replaced with the compact curve $C$. Again, a stratification argument shows that

$$
Z_{C}^{\text {top }}(q, a)=\left(1-q^{2}\right)^{1-\chi(C)} Z_{C, p}(q, a)
$$

respectively

$$
Z_{C}^{\mathrm{ref}}(q, a)=\prod_{k, w \geq 0}\left(\frac{1}{1-(-1)^{w} y^{k} q^{2}}\right)^{(-1)^{w} h_{c}^{k, w}(C \backslash\{p\})} Z_{C, p}^{\mathrm{ref}}(q, a, y)
$$

by analogy with the similar formulas in Section 1.2. The integers $h_{c}^{k, w}(C)$ $\{p\})$ are the weighted Betti numbers of $C \backslash\{p\}$ of compactly supported cohomology equipped with Deligne's weight filtration.

Then one of the main results of this paper is the following theorem for topological Euler character invariants. 


\section{Theorem 1.1.}

$$
Z^{\mathrm{top}}\left(X, C, q^{2},-a^{2}\right)=Z^{\mathrm{top}}\left(X, C_{0}, q^{2},-a^{2}\right) q^{2 \chi\left(\mathcal{O}_{C}\right)} Z_{C}^{\mathrm{top}}(q, a) .
$$

Theorem 1.1 follows from Proposition 1.2 and Theorem 1.3 below, which rely heavily on wallcrossing for framed stable pair invariants. The general framework is outlined in the next subsection, and presented in more detail in Section 2.

The motivic version of identity (1.12) will be discussed in Section 1.6, once the main steps in the proof of Equation (1.12) are clearly understood.

\section{4. $C$-framed perverse coherent sheaves and stability}

Let $D^{b}(X)$ be the bounded derived category of $X$. Let $\mathcal{A} \subset D^{b}(X)$ be the heart of the perverse $t$-structure on $D^{b}(X)$ determined by the torsion pair $\left(\mathrm{Coh}_{\geq 2}(X), \mathrm{Coh}_{\leq 1}(X)\right)$. The objects of $\mathcal{A}$ are objects $E$ of $D^{b}(Y)$ such that the cohomology sheaves $\mathcal{H}^{i}(E)$ are nontrivial only for $i=-1,0, \mathcal{H}^{-1}(E)$ has no torsion in codimension $\geq 2$, and $\mathcal{H}^{0}(E)$ is torsion, of dimension $\leq 1$. Let $\omega$ be a fixed Kähler class on $X$.

The stable pair theory of $X$ has been studied in $[50,51]$ employing a construction of limit (or weak) stability conditions on $\mathcal{A}$, which we review in Section 2.1. The main motivation for the study of limit stability conditions in the loc.cit. was to prove the rationality conjecture of Pandharipande and Thomas [45]. The main tool in the proof is the wall-crossing formalism of $[24,26]$ applied to a one-parameter family of stability conditions on $\mathcal{A}$ parameterized by a $B$-field, $B=b \omega \in H^{2}(X)$. In fact, as was pointed out in [26], the wall-crossing formulas for the weak stability conditions is a special case of those considered in the loc.cit. as soon as one allows the central charge to take values in an ordered field. Weak stability conditions are easy to construct $[50,51]$ for the derived category of coherent sheaves $D^{b}(X)$ on a Calabi-Yau manifold $X$, differently from conventional Bridgeland stability conditions. More specifically, there is a slope function $\mu_{(\omega, b)}$ on the Grothendieck group $K_{0}(\mathcal{A})$ which defines a family of weak stability conditions on $\mathcal{A}$, as reviewed in Section 2.1. Moreover, the following results are proven in [50].

1. For fixed $(\beta, n)$ there is an algebraic moduli stack of finite type $\mathcal{M}_{b}^{s s}(\mathcal{A}, \beta, n)$ of $\mu_{(\omega, b)}$-semistable objects of $\mathcal{A}$ with $\operatorname{ch}(E)=(-1,0, \beta, n)$.

2. For fixed $(\beta, n)$ there are finitely many critical parameters $b_{c}$ such that strictly $\mu_{(\omega, b)}$-semistable objects exist. The moduli stacks $\mathcal{M}_{\left(\omega, b^{\prime}\right)}^{s s}(\mathcal{A}, \beta, n)$, $\mathcal{M}_{\left(\omega, b^{\prime \prime}\right)}^{s s}(\mathcal{A}, \beta, n)$ are canonically isomorphic if there is no critical stability 
parameter in the interval $\left[b^{\prime}, b^{\prime \prime}\right]$. Moreover, if $b$ is not critical all closed points of $\mathcal{M}_{(\omega, b)}^{s s}(\mathcal{A}, \beta, n)$ are $\mu_{(\omega, b)}$-stable and their stabilizers are canonically isomorphic to $\mathbb{C}^{\times}$.

3. For fixed $\omega,(\beta, n)$, there exists $b_{-\infty}$ such that for any $b<b_{-\infty}$ the moduli stack $\mathcal{M}_{(\omega, b)}^{s s}(\mathcal{A}, \beta, n)$ is an $\mathbb{C}^{\times}$-gerbe over the moduli space $P(X, \beta, n)$ of stable pairs on $X$.

A similar construction will be employed in the proof of Theorem 1.1. A full subcategory $\mathcal{A}^{C}$ of $\mathcal{A}$ consisting of $C$-framed perverse coherent sheaves $\mathcal{A}^{C}$ is defined by conditions $(C .1),(C .2)$ in Section 2.2. Then it is shown that the slope construction of weak stability conditions [50] and basic properties of slope limit semistable objects carry over to the $C$-framed category. In particular, one can construct a one parameter family of weak stability conditions parameterized by the $B$-field $B=b \omega \in H^{2}(X)$.

The moduli stacks of $\mu_{(\omega, b)}$-semistable objects $E$ in $\mathcal{A}^{C}$ with numerical invariants $\operatorname{ch}(E)=\left(-1,0,[C]+r\left[C_{0}\right], n\right), r \in \mathbb{Z}_{\geq 0}, n \in \mathbb{Z}$, will be denoted by $\mathcal{P}_{(\omega, b)}(X, C, r, n)$. Their properties are completely analogous $(\mathbf{1})-(\mathbf{3})$ above. In particular, they are algebraic stacks of finite type, and for fixed $\omega$ and numerical invariants $(r, n)$ strictly semistable objects exist only for finitely many critical values of $b$. Moreover, there exists $b_{-\infty} \in \mathbb{R}_{<0}$ such that for $b<b_{-\infty} \mathcal{P}_{(\omega, b)}(X, C, r, n)$ is a $\mathbb{C}^{\times}$-gerbe over the moduli space of $C$-framed stable pairs.

Let $O b(\mathcal{A})$ be the stack of all objects of $\mathcal{A}$, which is an algebraic stack locally finite type over $\mathbb{C}$. For all $b \in \mathbb{R}$ and all $(r, n) \in \mathbb{Z}_{\geq 0} \times \mathbb{Z}$ the natural forgetful morphism

$$
\mathcal{P}_{(\omega, b)}(X, C, r, n) \hookrightarrow O b(\mathcal{A})
$$

determine a stack function in the motivic Hall algebra $H(\mathcal{A})$.

Counting invariants $P_{(\omega, b)}^{\nu}(X, C, r, n)$ are again defined by integration with respect to a suitable constructible function $\nu$ on the stack of all objects $O b(\mathcal{A})$. Let

$$
Z_{(\omega, b)}^{\nu}(X, C ; u, T)=\sum_{n \in \mathbb{Z}} \sum_{r \geq 0} P_{(\omega, b)}^{\nu}(X, C, r, n) u^{n} T^{r}
$$

denote the resulting generating series. When $\nu$ is a motivic weight function, the invariants $P_{(\omega, b)}(X, C, r, n)$ take values in a ring of motives, and refined invariants $P_{(\omega, b)}^{\mathrm{ref}}(X, C, r, n ; y)$ are obtained by taking virtual Poincaré polynomials. For future reference note that counting invariants for objects $E$ of $\mathcal{A}^{C}$ with $\operatorname{ch}(E)=\left(0,0, r\left[C_{0}\right], n\right)$ are defined analogously, and coincide with the counting invariants of the conifold [40,48], [24,37, Ex 6.2]. Their generating function will be denoted by $Z^{\nu}\left(X, C_{0}, u, T\right)$. Let us fix $\omega$ and 
$(r, n) \in \mathbb{Z}_{\geq 0} \times \mathbb{Z}$. The stability parameter $b>0$ will be called small if there are no critical stability parameters of type $(r, n)$ in the interval $(0, b]$. The corresponding invariants will be denoted by $P_{0+}^{\nu}(X, C, r, n)$, and their generating function, $Z_{0+}^{\nu}(X, C, u, T)$. Moreover, for $b \ll 0$, the corresponding invariants $P_{-\infty}^{\nu}(X, C, r, n)$ specialize to stable pair invariants. In the following the function $\nu$ will be either the constant function $\nu=1$ or the motivic weight function defined in [26, Section 6.2].

\subsection{Factorization via wallcrossing and small $b$ chamber}

The first step in the proof of Theorem 1.1 is the derivation of a wall-crossing formula relating $b \ll 0$ invariants to small $b>0$ invariants. More precisely, the following result is proven in Appendix A.

\section{Proposition 1.2.}

$$
Z_{-\infty}^{\mathrm{top}}(X, C, u, T)=Z^{\mathrm{top}}\left(X, C_{0}, u, T\right) Z_{0+}^{\mathrm{top}}(X, C, u, T) .
$$

This is in agreement with the natural factorization of Wilson loop expectation values in Chern-Simons theory, as we explained below Equation (1.5).

In order to complete the proof of Theorem 1.1., one has to find a connection between the moduli spaces of stable $C$-framed objects for small $b>0$ and the Hilbert scheme invariants (1.10). This is the content of Theorem 1.3 below, which follows from Propositions 3.6, 3.11.

Theorem 1.3. There is an identity of generating functions

$$
Z_{0+}^{\text {top }}\left(X, C ; q^{2},-a^{2}\right)=q^{2 \chi\left(\mathcal{O}_{C}\right)} Z_{C}^{\text {top }}(q, a),
$$

where $Z_{C}^{\text {top }}(q, a)$ is the series (1.10).

The proof of Theorem 1.3 relies on the construction in Section 3.2 of a moduli stack $\mathcal{Q}(X, C, r, n)$ of decorated sheaves on $X$ interpolating between the nested Hilbert schemes $H^{[l, r]}(C), l=n-\chi\left(\mathcal{O}_{C}\right)$ and the moduli stacks $\mathcal{P}_{0+}(X, C, r, n)$. More precisely, Proposition 3.6 proves that $\mathcal{Q}(X, C, r, n)$ is a $\mathbb{C}^{\times}$gerbe over a relative Quot scheme $Q^{[r, n]}(C)$ which is geometrically bijective to $H^{[r, n]}(C)$. At the same time Proposition 3.11 shows that $\mathcal{Q}(X, C, r, n)$ is equipped with a natural geometric bijection $f: \mathcal{Q}(X, C, r, n) \rightarrow \mathcal{P}_{0+}$ $(X, C, r, n)$. Then the proof of Theorem 1.3 reduces to a straightforward stratification computation explained in detail at the end of Section 3. 


\subsection{Generalization to motivic DT invariants}

A motivic version of identity (1.12) can be derived from the formalism of [26] following the same main steps. Assuming the required foundational results, the motivic wallcrossing formula of [26] implies the motivic version of the factorization formula (1.14), repeating the computations in A.2 and A.3. A similar computation using refined wall-crossing formulas has been carried for example in [7, Sect 2.4], hence the details will be omitted. This reduces the problem to the motivic analogue of Theorem 1.3.

The motivic DT theory of $C$-framed stable objects at small $b>0$ is analyzed in Section 4. As shown in Section 4.5, the following identity holds

$$
Z_{0+}^{\operatorname{mot}}\left(X, C ; q^{2} \mathbb{L}^{1 / 2}, a^{2}\right)=\mathbb{L}^{\left(1-k^{2}\right) / 2} q^{2 \chi\left(\mathcal{O}_{C}\right)} Z_{C}^{\operatorname{mot}}(q, a)
$$

provided that the virtual motive of the moduli stack $\mathcal{P}_{0+}(X, C, r, n)$ is related to the Chow motive by the formula

$$
\left[\mathcal{P}_{0+}(X, C, r, n)\right]^{v i r}=\mathbb{L}^{\left(r^{2}-k^{2}-n+1\right) / 2}\left[\mathcal{P}_{0+}(X, C, r, n)\right]
$$

where $k$ is the degree of the curve $C$ in $\mathbb{P}^{2}$. This formula is proven in Section 4 for sufficiently high degree $n \gg 0$, assuming the foundational aspects of the motivic DT theory of [26], as well as a specific choice of orientation data. For arbitrary values of $n \in \mathbb{Z}$, the Equation (1.17) reduces to a relation (4.5) between motivic weights of moduli stacks of pairs and sheaves for irreducible curve classes. This is a virtual motivic counterpart to [46, Thm. 4]. Motivated by this analogy, it is natural to conjecture that this relation equation holds for all $n \in \mathbb{Z}$ with a suitable choice of orientation data. Granting Equation (1.17), identity (1.16) follows from a stratification computation presented in Section 4.5 .

\subsection{Outlook and future directions}

This section records potential generalizations and extensions of the conjecture of Oblomkov and Shende motivated by the string theory construction of [11]. These are just possible future directions of study, not established mathematical results, or, in some cases, not even precise conjectures. Nevertheless, they are recorded here for the interested reader in the hope that they will lead to interesting developments at some point in the future.

1.7.1. BPS states and nested Jacobians As observed in Remarks 3.5, 3.8 , a second moduli space $M^{[l, r]}(C)$ naturally enters the picture, which can 
be identified with a moduli space of nested Jacobians. The closed points of $M^{[l, r]}(C)$ are pairs $(J, \psi)$ where $J$ is a rank one torsion-free sheaf on $C$ of degree $-l$, and $\psi: J \rightarrow \mathcal{O}_{p}^{\oplus r}$ a surjective morphism. According to Lemma 3.9 and Remark 3.10, allowing the curve $C$ to vary in the linear system $|k H|$ on $D$ results in a smooth moduli space $\mathcal{N}(D, k, r, n)$. Moreover, this moduli space is equipped with a natural determinant map

$$
h: \mathcal{M}(D, k, r, n) \rightarrow|k H|
$$

to the linear system and $M^{[l, r]}(C)$ is the fiber of $h$ at the point corresponding to $C$. Then physics arguments $[14,15]$ predict that the cohomology of $M^{[l, r]}(C)$ should admit a perverse sheaf decomposition

$$
H\left(M^{[l, r]}(C)\right) \simeq \oplus_{p} G r^{p} H\left(M^{[l, r]}(C)\right)
$$

determined by an $h$-relative ample class. Moreover, the dimensions of the perverse graded pieces, $N_{r}^{p}=\operatorname{dim} G r^{p} H\left(M^{[l, r]}(C)\right)$, should be independent of the polarization and $n$, and the $C$-framed small $b$ generating function $Z_{0+}(X, C ; u, T)$ should admit a Gopakumar-Vafa expansion

$$
Z_{0+}(X, C ; u, T)=\frac{\sum_{r \geq 1} \sum_{p} N_{r}^{p} T^{r} u^{p}}{(1-u)^{2}}
$$

Note that the $r=0$ version of these conjectures is a rigorous mathematical result by work of $[35,36,47]$. The construction sketched above provides a possible generalization for $r \geq 1$ which deserves further study.

1.7.2. A conjecture for colored HOMFLY polynomials Theorem 1.1 and the conjecture of Oblomkov and Shende imply that $C^{\circ}$-framed stable pairs on the conifold are related to the HOMFLY polynomial of the link of the singular point $p \in C^{\circ}$. Large $N$ duality arguments [11] lead to the following generalization.

Let $(x, y, z)$ be the affine local coordinates on $Y$ such that the projection $Y \rightarrow \mathbb{P}^{1}$ is locally given by $(x, y, z) \rightarrow z$ and $C^{\circ}$ is contained in the fiber $z=0$. Hence, $C^{\circ}$ is a complete intersection of the form

$$
z=0, \quad f(x, y)=0
$$

where $f$ is a degree $k \geq 1$ irreducible polynomial of two variables. 
Let $\mu$ be a Young diagram consisting of $m_{i}$ columns of height $h_{i} \in \mathbb{Z}_{\geq 1}$, where $1 \leq i \leq s$ and

$$
h_{1}>h_{2}>\cdots>h_{s}
$$

Let $C_{\mu}^{\circ}$ be the complete intersection on $Y$ determined by the equations

$$
z^{h_{i}} f(x, y)^{m_{1}+\cdots+m_{i-1}}=0, \quad 1 \leq i \leq s
$$

where by convention $m_{0}=0$. Note that $C_{\mu}^{\circ}$ is a nonreduced irreducible subscheme of $Y$ of pure dimension one.

In complete analogy with Sections 1.2 one can define $C_{\mu}^{\circ}$-framed stable pair invariants of $Y$ employing the framing condition $\operatorname{Ann}(F) \subset \mathcal{I}_{C_{\mu}^{\circ}}$. Let $P\left(Y, C_{\mu} ; r, n\right)$ denote the counting invariants obtained by taking the quasiclassical limit of motivic DT invariants of the ambient space $\mathcal{P}^{\operatorname{circ}}(\bar{Y}, \beta, n)$, where $\beta=\left[C_{\mu}\right]+r\left[C_{0}\right]$. Based on large $N$ duality, the generating function

$$
Z\left(Y, C_{\mu}^{\circ}, q^{2}, a^{2}\right)=\sum_{n \in \mathbb{Z}} \sum_{r \geq 0} q^{2 n} a^{2 r} P\left(Y, C_{\mu}^{\circ}, r, n\right)
$$

is expected to be related to the $\mu$-colored HOMFLY polynomial $P_{K, \mu}(q, a)$. More specifically, a relation of the form

$$
P_{K, \mu}(q, a)=a^{\alpha} q^{\beta} Z^{B}\left(Y, C_{\mu}, q^{2}, a^{2}\right) Z^{B}\left(Y, q^{2}, a^{2}\right)
$$

is expected to hold, for certain integral exponents $\alpha, \beta$, possibly depending on $\mu$.

\section{Framed stable pairs in the derived category}

\subsection{Review of slope limit stability}

This section is a brief review of limit slope stability conditions on the derived category of a smooth projective Calabi-Yau threefold following $[1,50,51]$.

Let $D^{b}(X)$ be the bounded derived category of $X$. Let $\mathcal{A}$ be the heart of the t-structure determined by the torsion pair $\left(\mathrm{Coh}_{\geq 2}(X), \mathrm{Coh}_{\leq 1}(X)\right)$. The objects of $\mathcal{A}$ are objects $E$ of $D^{b}(Y)$ such that the cohomology sheaves $\mathcal{H}^{i}(E)$ are nontrivial only for $i=-1,0, \mathcal{H}^{-1}(E)$ has no torsion in codimension $\geq 2$, and $\mathcal{H}^{0}(E)$ is torsion, of dimension $\leq 1$. 
Let $\omega$ be a Kähler class on $X$ and $B \in H^{2}(X)$, a real cohomology class i.e., a $B$-field. Let $\mathcal{Z}_{(\omega, B)}: K(X) \rightarrow \mathbb{C}$ be the central charge function

$$
\mathcal{Z}_{(\omega, B)}(E)=-\int_{X} \operatorname{ch}(E) \mathrm{e}^{-(B+\mathrm{i} \omega)} \sqrt{\operatorname{Td}(X)} .
$$

For any $m \in \mathbb{R}_{>0}$ let

$$
\mathcal{Z}_{(\omega, B)}^{\dagger}(E)=\left(\operatorname{Re} \mathcal{Z}_{(m \omega, B)}(E)\right)^{\dagger}+\mathrm{i}\left(\operatorname{Im} \mathcal{Z}_{(m \omega, B)}(E)\right)^{\dagger},
$$

where $f^{\dagger}(m)$ denotes the leading monomial of a polynomial $f(m)$. Then for $m>>0$ the following

$$
\mu_{(\omega, B)}(E)=-\frac{\left(\operatorname{Re}^{-\mathrm{i} \pi / 4} \mathcal{Z}_{(m \omega, B)}(E)\right)^{\dagger}}{\left(\operatorname{Im} \mathrm{e}^{-\mathrm{i} \pi / 4} \mathcal{Z}_{(m \omega, B)}(E)\right)^{\dagger}}
$$

is a well-defined map to the field of rational functions $\mathbb{R}(m)$.

An object $E$ of $\mathcal{A}$ is said to be $\mu_{(\omega, B)^{-}}$(semi)stable if any proper nonzero subobject $0 \subset F \subset E$ in $\mathcal{A}$ satisfies

$$
\mu_{(\omega, B)}(F)(\leq) \mu_{(\omega, B)}(E) .
$$

Here rational functions $f, g \in \mathbb{R}(m)$ are ordered by

$$
f \geq g \quad \Leftrightarrow \quad f(m) \geq g(m) \quad \forall m>>0 .
$$

According to [50] the above slope stability gives rise to a weak stability condition on $\mathcal{A}$.

In order to study the properties of semistable objects of $\mathcal{A}$, it is helpful to consider the following full subcategories $\left(\mathcal{A}_{1}, \mathcal{A}_{1 / 2}\right)$ of $\mathcal{A}$ (see $\left.[50,51]\right)$. The category $\mathcal{A}_{1} \subset \mathcal{A}$ consists of objects $E$ such that $\mathcal{H}^{-1}(E)$ is torsion and $\mathcal{H}^{0}(E)$ is zero-dimensional. By definition $\mathcal{A}_{1 / 2}$ is the subcategory of $\mathcal{A}$ consisting of objects $E$ such that $\operatorname{Hom}_{\mathcal{A}}\left(\mathcal{A}_{1}, E\right)=0$ (i.e., it is right orthogonal to $\mathcal{A}_{1}$ ). Note that $\mathcal{H}^{-1}(E)$ is torsion-free for all objects of $E$ of $\mathcal{A}_{1 / 2}$, and also $\operatorname{Hom}(T, E)=0$ for any zero-dimensional sheaf $T$. According to [51, Lemm. 2.16] the subcategories $\left(\mathcal{A}_{1}, \mathcal{A}_{1 / 2}\right)$ define a torsion pair in $\mathcal{A}$. A morphism $E \rightarrow F$ of objects in $\mathcal{A}_{i}, i=1,1 / 2$ will be called a strict monomorphism/epimorphism if it is injective/surjective as a morphism in $\mathcal{A}$, and its cokernel/kernel belongs to $\mathcal{A}_{i}$.

In the following we consider objects $E$ of $\mathcal{A}$ with $\operatorname{ch}_{0}(E)=-1$ and $\operatorname{ch}_{1}(E)=0$. The first observation following from [50, Lemm. 3.8] is that 
if such an object is $\mu_{(\omega, B)}$-semistable, then it must belong to $\mathcal{A}_{1 / 2}$. Moreover the following stability criterion holds [50, Prop. 3.13].

Proposition 2.1. An object $E$ of $\mathcal{A}_{1 / 2}$ with $\operatorname{ch}_{0}(E)=-1$ and $\operatorname{ch}_{1}(E)=0$ is $\mu_{(\omega, B)}$-(semi)stable if and only if the following hold.

(i) For any strict epimorphism $E \rightarrow G$ in $\mathcal{A}_{1 / 2}$, with $G$ a pure dimension one sheaf on $X$

$$
\mu_{(\omega, B)}(G)(\geq)-\frac{3 B \omega^{2}}{\omega^{3}} .
$$

(ii) For any strict monomorphism $F \hookrightarrow E$ in $\mathcal{A}_{1 / 2}$, with $F$ a pure dimension one sheaf on $X$,

$$
\mu_{(\omega, B)}(F)(\leq)-\frac{3 B \omega^{2}}{\omega^{3}} .
$$

Next let $\beta \in H_{2}(X)$ and $n \in \mathbb{Z}$. Suppose $B=b \omega, b \in \mathbb{R}$. Then the following results are proven in [50] for fixed $\omega,(\beta, n)$.

1. For any $b \in \mathbb{R}$, there is an algebraic moduli stack of finite type $\mathcal{M}_{(\omega, B)}^{\text {ss }}$ $(\mathcal{A}, \beta, n)$ of $\mu_{(\omega, B)}$-semistable objects of $\mathcal{A}$ with $\operatorname{ch}(E)=(-1,0, \beta, n)$.

2. There are finitely many critical parameters $b_{c}$ such that strictly $\left(\omega, B_{c}\right)$-semistable objects exist. The moduli stacks $\mathcal{M}_{\left(\omega, B^{\prime}\right)}^{s s}(\mathcal{A}, \beta, n)$, $\mathcal{M}_{\left(\omega, B^{\prime \prime}\right)}^{s s}(\mathcal{A}, \beta, n)$ are isomorphic if there is no critical stability parameter in the interval $\left[b^{\prime}, b^{\prime \prime}\right]$. Moreover, if $b$ is not critical all closed points of $\mathcal{M}_{(\omega, B)}^{s s}(\mathcal{A}, \beta, n)$ are $\mu_{(\omega, B)}$-stable and have $\mathbb{C}^{\times}$stabilizers.

3. There exists $b_{-\infty}$ such that for any $b<b_{-\infty}$ the moduli stack $\mathcal{M}_{(\omega, B)}^{s s}$ $(\mathcal{A}, \beta, n)$ is a $\mathbb{C}^{\times}$-gerbe over the the moduli space $\mathcal{P}(Y, \beta, n)$ of stable pairs on $Y$ constructed in [45].

4 One can define counting invariants and wall-crossing formulas using either the formalism of Joyce and Song or the one of Kontsevich and Soibelman. In particular, there is a Hall algebra of motivic stack functions associated to the abelian category $\mathcal{A}$. The corresponding wallcrossing formulas are in agreement with those of Kontsevich and Soibelman [26].

\subsection{A $C$-framed subcategory}

In this section, $X$ will be a small crepant resolution of a nodal CalabiYau threefold $X_{0}$ as in Section 1.4. In particular, it will be assumed that all conditions listed there are satisfied. Therefore there is only one conifold point lying on a Weil divisor $\Delta \simeq \mathbb{P}^{2} \subset X_{0}$. The exceptional locus of the blow-up map $X \rightarrow X_{0}$ consists of a single rational $(-1,-1)$ curve $C_{0} \subset X$ 
which intersects the strict transform $D \subset X$ of $\Delta$ transversely at a point $p$. Let $B=b \omega, b \in \mathbb{R}$, where $\omega$ is a fixed Kähler class on $X$ as above. Without loss of generality, it will be assumed from now on that $\omega$ is normalized such that $\int_{C_{0}} \omega=1$.

Let $C \subset X$ be a irreducible reduced plane curve contained in $D$ passing through the point $p$ of intersection between $D$ and $C_{0}$. Consider the full subcategory $\mathcal{A}^{C}$ of $\mathcal{A}$ consisting of objects $E$ satisfying the conditions

(C.1) $\mathcal{H}^{-1}(E)$ is a subsheaf of the defining ideal $\mathcal{I}_{C}$. In particular, if $\mathcal{H}^{-1}(E)$ is not trivial, it must be the ideal sheaf of a proper closed subscheme $Z_{E} \subset X$.

(C.2) The structure sheaf $\mathcal{O}_{Z_{E}}$ and the cohomology sheaf $\mathcal{H}^{0}(E)$ are topologically supported on the union $C \cup C_{0}$. Moreover, the quotient $\mathcal{H}^{0}(E) / Q$ is topologically supported to $C_{0}$, where $Q \subset \mathcal{H}^{0}(E)$ is the maximal dimension zero subsheaf.

Lemma 2.2. Consider an exact sequence

$$
0 \rightarrow F \rightarrow E \rightarrow G \rightarrow 0
$$

in $\mathcal{A}$ where $\operatorname{ch}_{0}(E) \in\{0,-1\}$. Then the following statements hold

(i) If F, $G$ belong to $\mathcal{A}^{C}$ and then $E$ belongs to $\mathcal{A}^{C}$.

(ii) If $F, E$ belong to $\mathcal{A}^{C}$ then $G$ belongs to $\mathcal{A}^{C}$.

(iii) If $E, G$ belong to $\mathcal{A}^{C}$ then $F$ belongs to $\mathcal{A}^{C}$.

Proof. The above statements are obvious if $\operatorname{ch}_{0}(E)=0$ since then $\operatorname{ch}_{0}(F)=$ $\operatorname{ch}_{0}(G)=0$ and (2.1) is a sequence of sheaves on $X$.

Suppose $\operatorname{ch}_{0}(E)=-1$, which implies $\operatorname{ch}_{0}(F)=0, \operatorname{ch}_{0}(G)=-1$ or $\operatorname{ch}_{0}(F)=-1, \operatorname{ch}_{0}(G)=0$. Then all the above statements follow easily from the long exact sequence

$$
0 \rightarrow \mathcal{H}^{-1}(F) \rightarrow \mathcal{H}^{-1}(E) \rightarrow \mathcal{H}^{-1}(G) \rightarrow \mathcal{H}^{0}(F) \rightarrow \mathcal{H}^{0}(E) \rightarrow \mathcal{H}^{0}(G) \rightarrow 0
$$

except case $(i i), \operatorname{ch}_{0}(G)=-1, \operatorname{ch}_{0}(F)=0$, which requires more work. In this case, $\mathcal{H}^{-1}(F)=0$ and $\mathcal{H}^{-1}(G)$ is a rank one sheaf on $X$ which admits torsion at most in codimension one by the definition of $\mathcal{A}$. Therefore the maximal torsion subsheaf $T \subset \mathcal{H}^{-1}(G)$ is either zero or a nontrivial pure dimension two sheaf on $X$. Below it will be shown that $T$ must be zero.

Suppose $T$ is a nontrivial pure dimension two sheaf, and let $I \subseteq \mathcal{H}^{0}(F)$ denote the image of $T$ in $\mathcal{H}^{0}(F)$. By assumption, $\mathcal{H}^{0}(F)$ has topological 
support on $C \cup C_{0}$ since $F$ belongs to $\mathcal{A}^{C}$. Therefore $I$ has at most $1 \mathrm{D}$ support. Let $K$ be the kernel of the induced surjective morphism $T \rightarrow I$. Then $K$ must be a nontrivial sheaf of pure dimension two as well. Next note that there is a commutative diagram

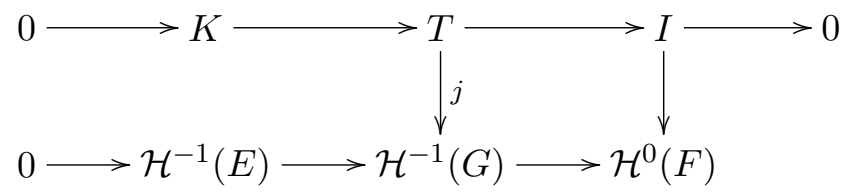

where the top row is exact, the bottom row is exact at the first two terms, and the vertical arrows are injective. Then the image of the restriction $\left.j\right|_{K}$ is a subsheaf of $\mathcal{H}^{-1}(E)$ and the snake lemma implies that $\operatorname{Ker}\left(\left.j\right|_{K}\right) \subset \operatorname{Ker}(j)$, which is trivial. Therefore $\operatorname{Ker}\left(\left.j\right|_{K}\right)=0$, which implies that $K$ is a subsheaf of $\mathcal{H}^{-1}(E)$. However, by assumption, $E$ belongs to $\mathcal{A}^{C}$ and has rank -1 , hence $\mathcal{H}^{-1}(E)$ is a torsion free sheaf of rank 1 . This implies that $K$ must be trivial, leading to a contradiction.

In conclusion, $T$ is trivial, hence $\mathcal{H}^{-1}(G)$ must be a rank one torsion free sheaf. Moreover, the exact sequence (2.2) implies under the current assumptions that $\mathcal{H}^{-1}(G)$ must have trivial determinant i.e., it must be isomorphic to the ideal sheaf of a closed subscheme $Z_{G}$ on $X$ of dimension at most one. There is also an inclusion $\mathcal{H}^{-1}(E) \hookrightarrow \mathcal{H}^{-1}(G)$ which implies that $Z_{G}$ is a closed subscheme of $Z_{E}$ and a simple application of the snake lemma yields an isomorphism

$$
K=\mathcal{H}^{-1}(G) / \mathcal{H}^{-1}(E) \simeq \operatorname{Ker}\left(\mathcal{O}_{Z_{E}} \rightarrow \mathcal{O}_{Z_{G}}\right) .
$$

Since $K \subset \mathcal{H}^{0}(F)$ and both $\mathcal{O}_{Z_{E}}$ and $\mathcal{H}^{0}(F)$ are topologically supported on $C \cup C_{0}$, it follows that $\mathcal{O}_{Z_{G}}$ satisfies the same condition. Moreover, in the exact sequence $(2.2), \mathcal{H}^{0}(F), \mathcal{H}^{0}(E)$ satisfy condition (C.2), which implies that $\mathcal{H}^{0}(G)$ also satisfies (C.2). Finally note that $K \subset \mathcal{H}^{0}(F)$ is topologically supported on a union of $C_{0}$ and a finite set of closed points lying on $C$. Therefore $\operatorname{Hom}_{X}\left(K, \mathcal{O}_{C}\right)=0$ since $\mathcal{O}_{C}$ is pure dimension one by assumption. This implies that the canonical projection $\mathcal{O}_{Z_{E}} \rightarrow \mathcal{O}_{C}$ factors through $\mathcal{O}_{Z_{E}} \rightarrow \mathcal{O}_{Z_{G}}$ i.e., there is a surjective morphism $\mathcal{O}_{Z_{G}} \rightarrow \mathcal{O}_{C}$ such that the diagram

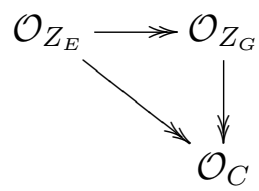

is commutative. Hence $\mathcal{H}^{-1}(G)$ is a subsheaf of $\mathcal{I}_{C}$. 
Limit slope stability for objects of $\mathcal{A}^{C}$ will be defined by analogy with $[50$, 51]. An object $E$ of $\mathcal{A}^{C}$ is $(\omega, B)$-(semi)stable if

$$
\mu_{(\omega, B)}(F)(\leq) \mu_{(\omega, B)}(E)
$$

for any proper nontrivial subobject $0 \subset F \subset E$ in $\mathcal{A}^{C}$. Since the Kähler class $\omega$ will be fixed, and $B=b \omega$ with $b \in \mathbb{R}$, the slope $\mu_{(\omega, B)}$ will be denoted by $\mu_{(\omega, b)}$. Moreover, $(\omega, B)$-limit slope (semi)stable objects of $\mathcal{A}^{C}$ will be called simply $\mu_{(\omega, b)}$-(semi)stable when the meaning is clear from the context.

Let $\mathcal{A}_{i}^{C}$ be the full subcategories of $\mathcal{A}^{C}$ consisting of objects belonging to $\mathcal{A}_{i}, i=1,1 / 2$. Given the definition of $\mathcal{A}^{C}$, it follows that $\mathcal{A}_{1}^{C}$ is the subcategory of zero-dimensional subsheaves with topological support on $C \cup C_{0}$. Let $E$ be an object of $\mathcal{A}^{C}$. Since the pair $\left(\mathcal{A}_{1}, \mathcal{A}_{1 / 2}\right)$ is a torsion pair in $\mathcal{A}$ (see [51]), there is an exact sequence

$$
0 \rightarrow E_{1} \rightarrow E \rightarrow E_{1 / 2} \rightarrow 0
$$

in $\mathcal{A}$ with $E_{i}$ in $\mathcal{A}_{i}, i=1,1 / 2$. Then the following holds

Lemma 2.3. Let $E$ be an object of $\mathcal{A}^{C}$. Then $E_{i}$ belongs to $\mathcal{A}_{i}^{C}, i=1,1 / 2$.

Proof. Consider again the exact sequence

$$
\begin{aligned}
0 & \rightarrow \mathcal{H}^{-1}\left(E_{1}\right) \rightarrow \mathcal{H}^{-1}(E) \rightarrow \mathcal{H}^{-1}\left(E_{1 / 2}\right) \rightarrow \mathcal{H}^{0}\left(E_{1}\right) \\
& \rightarrow \mathcal{H}^{0}(E) \rightarrow \mathcal{H}^{0}\left(E_{1 / 2}\right) \rightarrow 0
\end{aligned}
$$

By definition, $\mathcal{H}^{-1}\left(E_{1}\right)$ must be a torsion sheaf of dimension two, hence it must be trivial since $\mathcal{H}^{-1}(E)$ is torsion free. Therefore $E_{1} \simeq \mathcal{H}^{0}\left(E_{1}\right)$ must be a zero-dimensional sheaf. Let $I \subset \mathcal{H}^{0}(E)$ denote its image in $\mathcal{H}^{0}(E)$ and $K=\operatorname{Ker}\left(\mathcal{H}^{0}\left(E_{1}\right) \rightarrow I\right)$. Then there is an exact sequence of sheaves

$$
0 \rightarrow \mathcal{H}^{-1}(E) \rightarrow \mathcal{H}^{-1}\left(E_{1 / 2}\right) \rightarrow K \rightarrow 0
$$

Note that both $I$ and $K$ are zero-dimensional sheaves and $I$ is topologically supported on $C \cup C_{0}$. Suppose there exists a subsheaf $K^{\prime} \subset K$ with support disjoint from $C, C_{0}$. Since $\mathcal{H}^{-1}(E)=\mathcal{I}_{Z_{E}}$, and $Z_{E}$ is topologically supported on $C \cup C_{0}$, it follows that

$$
\mathcal{E} x t_{X}^{1}\left(K^{\prime}, \mathcal{H}^{-1}(E)\right) \simeq \mathcal{E} x t_{X}^{1}\left(K^{\prime}, \mathcal{O}_{X}\right) .
$$

However, [19, Prop. 1.1.6] shows that $\mathcal{E} x t_{X}^{1}\left(K^{\prime}, \mathcal{O}_{X}\right)=0$ since $K^{\prime}$ is zerodimensional. Therefore, using the local to global spectral sequence, 
$\operatorname{Ext}_{X}^{1}\left(K^{\prime}, \mathcal{H}^{-1}(E)\right)=0$, which implies that there is an injection $K^{\prime} \hookrightarrow \mathcal{H}^{-1}$ $\left(E_{1 / 2}\right)$. This leads to a contradiction since $\mathcal{H}^{-1}\left(E_{1 / 2}\right)$ cannot have zerodimensional torsion by construction. In conclusion, $K$, hence also $\mathcal{H}^{0}\left(E_{1}\right)$, is topologically supported on $C \cup C_{0}$. In particular, $E_{1}$ belongs to $\mathcal{A}^{C}$. Then Lemma 2.2 implies that $E_{1 / 2}$ must also belong to $\mathcal{A}^{C}$.

A consequence of Lemma 2.3 is that properties of limit slope semistable objects in $\mathcal{A}$ proven in $[50,51]$ also hold in $\mathcal{A}^{C}$. More specifically, strict monomorphisms and epimorphisms of objects in $\mathcal{A}_{i}^{C}, i=1,1 / 2$ may be defined again by requiring that the cokernel, respectively kernel belong to $\mathcal{A}_{i}^{C}$. Then, by analogy with [51, Lemm. 2.27], [50, Lemm. 3.8], it follows again that any $\mu_{(\omega, b)}$-(semi)stable object of $\mathcal{A}^{C}$ with $\operatorname{ch}_{0}(E)=-1$, must belong to $\mathcal{A}_{1 / 2}^{C}$. Moreover, the stability criterion in Proposition 2.1 holds for objects of $\mathcal{A}_{1 / 2}^{C}$ provided that $F \hookrightarrow E, E \rightarrow G$ are strict monomorphisms, respectively, epimorphisms in $\mathcal{A}_{1 / 2}^{C}$.

Some more specific properties of limit slope semistable objects in $\mathcal{A}^{C}$ are recorded below.

\subsection{Properties of $C$-framed limit slope stable objects}

First note that any nontrivial object $E$ of $\mathcal{A}^{C}$ with $\operatorname{ch}_{0}(E)=0$ must be a sheaf with topological support on $C \cup C_{0}$ and $\operatorname{ch}_{2}(E)=r\left[C_{0}\right], r \geq 0$. Moreover, if $r \geq 1$,

$$
\mu_{(\omega, b)}(E)=\frac{\chi(E)}{r}-b .
$$

Therefore, $(\omega, b)$-stability for such objects reduces to $\omega$-slope stability for dimension one sheaves on $X$. For completeness recall that a sheaf $E$ as above with $r \geq 1$ is $\omega$-slope (semi)stable if

$$
\left\langle\omega, \operatorname{ch}_{2}(E)\right\rangle \chi\left(E^{\prime}\right)(\leq)\left\langle\omega, \operatorname{ch}_{2}\left(E^{\prime}\right)\right\rangle \chi(E)
$$

for any nontrivial proper subsheaf $0 \subset E^{\prime} \subset E$. Since in the present case $\operatorname{ch}_{2}(E)=r\left[C_{0}\right], \operatorname{ch}_{2}\left(E^{\prime}\right)=r^{\prime}\left[C_{0}\right]$ for some $r, r^{\prime} \in \mathbb{Z}_{\geq 0}$, and $\omega$ is normalized such that $\int_{C_{0}} \omega=1$, this condition reduces to

$$
r \chi\left(E^{\prime}\right)(\leq) r^{\prime} \chi(E)
$$

Since any $\omega$-slope semistable sheaf must be pure, the defining property $(C .2)$ implies that any $\mu_{(\omega, b)}$-semistable object of $\mathcal{A}^{C}$ must be a pure dimension one sheaf with set theoretic support on $C_{0}$. Then note the following lemma. 
Lemma 2.4. Let $F$ be an $\omega$-slope semistable sheaf supported on $C_{0}$ with $\mathrm{ch}_{2}(F)=r C_{0}, r \geq 1$. Then $F$ is the extension by zero of a semistable locally free sheaf on $C_{0}$.

Proof. By construction the morphism $X \rightarrow X_{0}$ contracts $C_{0}$ to an ordinary double point, which is analytically isomorphic to the hypersurface singularity $x z-y w=0$ in $\mathbb{C}^{4}$. Therefore the formal neighborhood of $C_{0}$ in $X$ is isomorphic to the formal neighborhood of the zero section in the total space $Y$ of $\mathcal{O}_{\mathbb{P}^{1}}^{\oplus 2} \rightarrow \mathbb{P}^{1}$. Since any sheaf $F$ as in Lemma (2.4) is set theoretically supported in this neighborhood, it suffices to prove Lemma (2.4) for sheaves on $Y$ with topological support on the zero section. Abusing notation, throughout the proof the zero section of $Y \rightarrow \mathbb{P}^{1}$ will also be denoted by $C_{0}$.

Any sheaf $F$ on $Y$ with topological support on $C_{0}$ has $\operatorname{ch}_{2}(F)=r\left[C_{0}\right]$. Then slope semistability is defined by the condition

$$
r \chi\left(F^{\prime}\right) \leq r^{\prime} \chi(F)
$$

for any proper nontrivial subsheaf $0 \subset F^{\prime} \subset F$.

Let $\pi: Y \rightarrow \mathbb{P}^{1}$ denote the canonical projection, and let $\mathcal{O}_{Y}(-1)=\pi^{*}$ $\mathcal{O}_{\mathbb{P}^{1}}(-1)$. Then note that $C_{0} \subset Y$ is a complete intersection

$$
s_{1}=s_{2}=0,
$$

where $s_{1}, s_{2}$ are sections of $\mathcal{O}_{Y}(-1)$.

Now let $F$ be a slope semistable sheaf on $Y$ with topological support on $C_{0}$. Suppose one of the morphisms

$$
F \stackrel{s_{i} \otimes 1_{F}}{\longrightarrow} F \otimes_{Y} \mathcal{O}_{Y}(-1)
$$

is nonzero for some $i=1,2$. Let $G \subset F \otimes_{Y} \mathcal{O}_{Y}(-1)$ denote its image and $K \subset F$ its kernel. Both $G, K$ are pure of dimension one with $\operatorname{ch}_{2}(G)=$ $r_{G}\left[C_{0}\right], \operatorname{ch}_{2}(K)=r_{K}\left[C_{0}\right], r_{G}, r_{K} \geq 1, r_{G}+r_{K}=r$. Then

$$
\frac{\chi(G)}{r_{G}} \geq \frac{\chi(F)}{r}, \quad \text { and } \quad \frac{\chi(K)}{r_{K}} \leq \frac{\chi(F)}{r} .
$$

Moreover, it is straightforward to show that $F \otimes_{Y} \mathcal{O}_{Y}(-1)$ must be slope semistable as well and $\chi\left(F \otimes_{Y} \mathcal{O}_{Y}(-1)\right)=\chi(F)-r$. Therefore

$$
\frac{\chi(G)}{r_{G}} \leq \frac{\chi(F)}{r}-1,
$$

which leads to a contradiction. In conclusion $s_{i} \otimes 1_{F}=0$ for both $i=1,2$. 
Another simple class of objects of $\mathcal{A}^{C}$ are stable pairs $E=\left(\mathcal{O}_{X} \stackrel{s}{\longrightarrow} F\right)$ with $F$ a pure dimension one sheaf supported on $C_{0}$ and $\operatorname{Coker}(s)$ zerodimensional. With the present conventions, $\mathcal{O}_{X}, F$ are in degrees $-1,0$ respectively, unlike [45], where they have degrees 0,1 . The following result will be useful later.

Lemma 2.5. Suppose $E=\left(\mathcal{O}_{X} \stackrel{s}{\longrightarrow} F\right)$ is a stable pair on $X$ with $F$ a pure dimension one sheaf with topological support on $C_{0}$ and $\operatorname{ch}_{2}(F)=r\left[C_{0}\right], r \geq$ 1. Then $\chi(F) \geq r(r+1) / 2$.

Proof. Let $Z_{F} \subset X$ be the scheme theoretic support of $F$. Since $F$ is topologically supported on $C_{0}, Z_{F}$ must be a thickening of $C_{0}$. Moreover, there is an exact sequence

$$
0 \rightarrow \mathcal{O}_{Z_{F}} \stackrel{\left.s\right|_{Z_{F}}}{\longrightarrow} F \rightarrow Q \rightarrow 0
$$

with $Q$ zero-dimensional. This implies that $\chi(F) \geq \chi\left(\mathcal{O}_{Z_{F}}\right)$ and $\operatorname{ch}_{2}\left(\mathcal{O}_{Z_{F}}\right)=$ $\operatorname{ch}_{2}(F)=r\left[C_{0}\right]$ for some $r \geq 1$. Note that $\mathcal{O}_{Z_{F}}$ must be pure of dimension one since $F$ is so by assumption.

In order to conclude the proof it will be shown inductively in $r \geq 1$ that $\chi\left(\mathcal{O}_{Z}\right) \geq r(r+1) / 2$ for any pure dimension one $Z$ thickening of $C_{0}$ with $\operatorname{ch}_{2}\left(\mathcal{O}_{Z}\right)=r\left[C_{0}\right]$. By analogy with the proof of Lemma (2.4), it suffices to prove this for the zero section $C_{0} \subset Y$ of the total space $Y$ of the rank two bundle $\mathcal{O}_{\mathbb{P}^{1}}(-1)^{\oplus 2}$. Then $C_{0}$ is a complete intersection

$$
s_{1}=s_{2}=0
$$

where $s_{1}, s_{2}$ are sections of $\mathcal{O}_{Y}(-1)=\pi^{*} \mathcal{O}_{\mathbb{P}}^{-1}$.

The first step, $r=1$, is clear since in that case $\chi\left(\mathcal{O}_{Z}\right)=\chi\left(\mathcal{O}_{C_{0}}\right)=1$. Let $r \geq 2$ and suppose the statement is true for any $1 \leq r^{\prime} \leq r$.

First prove that for any pure dimension one thickening $Z$ of $C_{0}$ with $\operatorname{ch}_{2}\left(\mathcal{O}_{Z}\right)=r\left[C_{0}\right]$ there exists a section $s_{1}^{k_{1}} s_{2}^{k_{2}}$ of $\mathcal{O}_{Y}(-r)$, with $k_{1}, k_{2} \geq 0$, $k_{1}+k_{2}=r$ such that the morphism

$$
\zeta\left(k_{1}, k_{2}\right): \mathcal{O}_{Z} \otimes_{Y} \mathcal{O}_{Y}(r) \rightarrow \mathcal{O}_{Z}
$$

given by multiplication by $s_{1}^{k_{1}} s_{2}^{k_{2}}$ is nonzero and $\operatorname{ch}_{2}\left(\operatorname{Coker}\left(\zeta\left(k_{1}, k_{2}\right)\right)\right)=$ $r^{\prime}\left[C_{0}\right]$ for some $r^{\prime}>0$.

If all morphisms $\zeta\left(k_{1}, k_{2}\right), k_{1}, k_{2} \geq 0, k_{1}+k_{2}=r$ are trivial, it is easy to show that $\operatorname{ch}_{2}\left(\mathcal{O}_{Z}\right) \leq r-1$, leading to a contradiction. Therefore, at least one of them, $\zeta\left(j_{1}, j_{2}\right)$ must be nontrivial. Let $I=\operatorname{Im}\left(\zeta\left(j_{1}, j_{2}\right)\right)$ and 
$G=\operatorname{Coker}\left(\zeta\left(j_{1}, j_{2}\right)\right)$ and let

$$
\operatorname{ch}_{2}(I)=r_{I}\left[C_{0}\right], \quad \operatorname{ch}_{2}(G)=r_{G}\left[C_{0}\right]
$$

with $r_{I}, r_{G} \geq 0, r_{I}+r_{G}=r$.

Suppose $r_{G}=0$, that is $G$ is zero-dimensional, possibly trivial. Then $r_{I}=r$ and the kernel of $\zeta\left(j_{1}, j_{2}\right)$ must be a zero-dimensional sheaf. Hence $\zeta\left(j_{1}, j_{2}\right)$ must be injective since $\mathcal{O}_{Z} \otimes_{Y} \mathcal{O}_{Y}(-r)$ is pure of dimension one. Therefore

$$
\chi(G)=\chi\left(\mathcal{O}_{Z}\right)-\chi\left(\mathcal{O}_{Z} \otimes_{Y} \mathcal{O}_{Y}(r)\right)=-r^{2}<0,
$$

which is a contradiction. In conclusion $r_{G}>0$, hence $0<r_{I}, r_{G}<r$.

Let $T(G) \subset G$ be the maximal dimension zero subsheaf of $G$. Then $G / T(G)$ is a pure dimension one quotient of $\mathcal{O}_{Z}$, hence it must be the structure sheaf of a thickening of $C_{0}$. Using the inductive hypothesis,

$$
\chi(G) \geq \chi(G / T(G)) \geq \frac{1}{2} r_{G}\left(r_{G}+1\right) .
$$

Similarly, $I \otimes_{Y} \mathcal{O}_{Y}(-r)$ is a pure dimension one quotient of $\mathcal{O}_{Z}$ with $r_{I}<r$. Therefore, the induction hypothesis implies

$$
\chi\left(I \otimes_{Y} \mathcal{O}_{Y}(-r)\right) \geq \frac{1}{2} r_{I}\left(r_{I}+1\right)
$$

which yields

$$
\chi(I) \geq r_{I} r+\frac{1}{2} r_{I}\left(r_{I}+1\right) .
$$

In conclusion, since $r_{G}=r-r_{I}$,

$$
\begin{aligned}
\chi\left(\mathcal{O}_{Z}\right) & =\chi(G)+\chi(I) \geq r_{I} r+\frac{1}{2} r_{I}\left(r_{I}+1\right)+\frac{1}{2} r_{G}\left(r_{G}+1\right) \\
& =\frac{1}{2} r(r+1)+\frac{1}{2} r_{I}^{2} \geq \frac{1}{2} r(r+1) .
\end{aligned}
$$

In order to derive similar structure results for more general $\mu_{(\omega, b)^{-}}$ semistable objects, it will be helpful to note the following technical result.

Lemma 2.6. Let $F_{C}$ be a pure dimension one sheaf on $X$ with scheme theoretic support on $C$ and $F_{0}$ a pure dimension one sheaf on $X$ with topological support on $C_{0}$. Recall that the curve $C$, hence also the sheaf $F_{C}$, is scheme theoretically supported on a divisor $D \simeq \mathbb{P}^{2}$ in $X$ which intersects 
$C_{0}$ transversely at one point. Then, choosing a trivialization of the dualizing sheaf of $D$ at $p,\left.\omega_{D}\right|_{p} \simeq \mathcal{O}_{p}$, there are isomorphisms

$$
\varphi_{k}: \operatorname{Ext}_{X}^{k}\left(F_{C}, F_{0}\right) \stackrel{\sim}{\longrightarrow} \operatorname{Ext}_{D}^{k-1}\left(F_{C}, \mathcal{O}_{D} \otimes_{X} F_{0}\right)
$$

for all $k \in \mathbb{Z}$, where $\operatorname{Ext}_{D}^{k}$ are global extension groups of $\mathcal{O}_{D}$-modules. Moreover, suppose

$$
0 \rightarrow F_{0} \rightarrow F \rightarrow F_{C} \rightarrow 0
$$

is an extension of $\mathcal{O}_{X}$-modules corresponding to an extension class $e \in$ $\operatorname{Ext}_{X}^{1}\left(F_{C}, F_{0}\right)$ and let $F_{C}^{\prime} \subset F_{C}$ be a subsheaf of $F_{C}$. Then $e$ is in the kernel of the natural map

$$
\operatorname{Ext}_{X}^{1}\left(F_{C}, F_{0}\right) \rightarrow \operatorname{Ext}_{X}^{1}\left(F_{C}^{\prime}, F_{0}\right)
$$

if and only if $F_{C}^{\prime} \subset \operatorname{Ker}\left(\varphi_{1}(e)\right)$, where we consider $\varphi_{1}(e)$ to be a map $F_{C} \rightarrow$ $\left.F_{0}\right|_{D}$ of $\mathcal{O}_{D}$-modules.

Proof. The adjunction formula the canonical embedding $i: D \hookrightarrow X$ yields a quasi-isomorphism

$$
\operatorname{RHom}_{X}\left(F_{C}, F_{0}\right) \simeq \operatorname{RHom}_{D}\left(F_{C}, i^{!} F_{0}\right)
$$

where

$$
i^{!} F_{0}=L i^{*} F_{0} \otimes \omega_{D}[-1] .
$$

Note that the cohomology sheaves of the complex $L i^{*} F_{0}$ are isomorphic to the local tor sheaves

$$
\mathcal{H}^{k}\left(L i^{*} F_{0}\right) \simeq \mathcal{T}_{o r}^{X}\left(F_{0}, \mathcal{O}_{D}\right)
$$

for all $k \in \mathbb{Z}$. Moreover local tor is symmetric in its arguments, that is

$$
\mathcal{T}_{o r_{-k}^{X}}^{X}\left(F_{0}, \mathcal{O}_{D}\right) \simeq \mathcal{T}_{o r_{-k}^{X}}^{X}\left(\mathcal{O}_{D}, F_{0}\right)
$$

Since $F_{0}$ is pure of dimension one, using the canonical locally free resolution

$$
\mathcal{O}_{X}(-D) \stackrel{\zeta_{D}}{\longrightarrow} \mathcal{O}_{X}
$$

of $\mathcal{O}_{D}$, it follows that $\mathcal{T}_{o r}^{X}{ }_{-k}\left(\mathcal{O}_{D}, F_{0}\right)=0$ for all $k \neq 0$. Therefore, the complex $L i^{*} F_{0}$ is quasi-isomorphic to the sheaf $\mathcal{T}_{\text {or }}^{X}\left(\mathcal{O}_{D}, F_{0}\right) \simeq \mathcal{O}_{D} \otimes_{X} F_{0}$. Then (2.6) yields isomorphisms of the form (2.4). 
The second statement follows from the functoriality of the adjunction formula.

For future reference note the following corollary of Lemma 2.6.

Corollary 2.7. Under the same conditions as in Lemma 2.6 suppose $F_{0}=$ $V \otimes \mathcal{O}_{C_{0}}(-1)$ with $V$ a finite-dimensional vector space and let $e \in \operatorname{Ext}_{X}^{1}$ $\left(F_{C}, F_{0}\right)$ be an extension class. Let $\psi=\varphi_{1}(e) \in \operatorname{Hom}_{D}\left(F_{C}, V \otimes \mathcal{O}_{p}\right)$ be the corresponding morphism of $\mathcal{O}_{D}$-modules, where $\mathcal{O}_{p}$ is the structure sheaf of the transverse intersection point $\{p\}=D \cap C_{0}$. Then the following conditions are equivalent

(a) The class $e$ is not in the kernel of the natural map

$$
q_{*}: \operatorname{Ext}_{X}^{1}\left(F_{C}, V \otimes \mathcal{O}_{C_{0}}(-1)\right) \rightarrow \operatorname{Ext}_{X}^{1}\left(F_{C}, V^{\prime} \otimes \mathcal{O}_{C_{0}}(-1)\right),
$$

for any nontrivial quotient $q: V \rightarrow V^{\prime}$.

(b) The morphism $\psi: F_{C} \rightarrow V \otimes \mathcal{O}_{p}$ is surjective.

Proof. Suppose an extension class $e$ satisfies condition $(a)$ and $\psi$ is not surjective. Then the image of $\psi$ is $V^{\prime \prime} \otimes \mathcal{O}_{p}$ where $V^{\prime \prime} \subset V$ is a proper subspace of $V$. Let $V \simeq V^{\prime} \oplus V^{\prime \prime}$ be a direct sum decomposition, and $q: V \rightarrow V^{\prime}$ the natural projection. Then the second part of Lemma 2.6 implies that such that $e$ lies in the kernel of the map $q_{*}$, leading to a contradiction. The proof of the converse statement is analogous.

Now let $E$ be an object of $\mathcal{A}^{C}$ with $\operatorname{ch}_{0}(E)=-1$ and let $F$ be a torsion sheaf on $X$ of dimension at most one. Then there is an exact sequence

$$
\begin{aligned}
0 & \rightarrow \operatorname{Ext}_{X}^{1}\left(F, \mathcal{H}_{E}^{-1}\right) \rightarrow \operatorname{Hom}_{D^{b}(X)}(F, E) \rightarrow \operatorname{Ext}_{X}^{0}\left(F, \mathcal{H}^{0}(E)\right) \\
& \rightarrow \operatorname{Ext}_{X}^{2}\left(F, \mathcal{H}^{-1}(E)\right) \rightarrow \cdots
\end{aligned}
$$

Moreover, since $\mathcal{H}^{-1}(E)=\mathcal{I}_{Z_{E}}$ is the ideal sheaf of a dimension one subscheme, there is also an exact sequence

$$
0 \rightarrow \operatorname{Ext}_{X}^{0}\left(F, \mathcal{O}_{Z_{E}}\right) \rightarrow \operatorname{Ext}_{X}^{1}\left(F, \mathcal{H}^{-1}(E)\right) \rightarrow \operatorname{Ext}_{X}^{2}\left(F, \mathcal{O}_{X}\right) \rightarrow \cdots
$$

Lemma 2.8. Suppose $E$ is an object of $\mathcal{A}_{1 / 2}^{C}$ with $\operatorname{ch}_{0}(E)=-1, \operatorname{ch}_{2}(E)=$ $[C]+r\left[C_{0}\right], r \geq 0$. Then the following hold

(i) $Z_{E}$ is a pure dimension one subscheme of $X$. 
(ii) There is a commutative diagram of morphisms of $\mathcal{O}_{X}$-modules

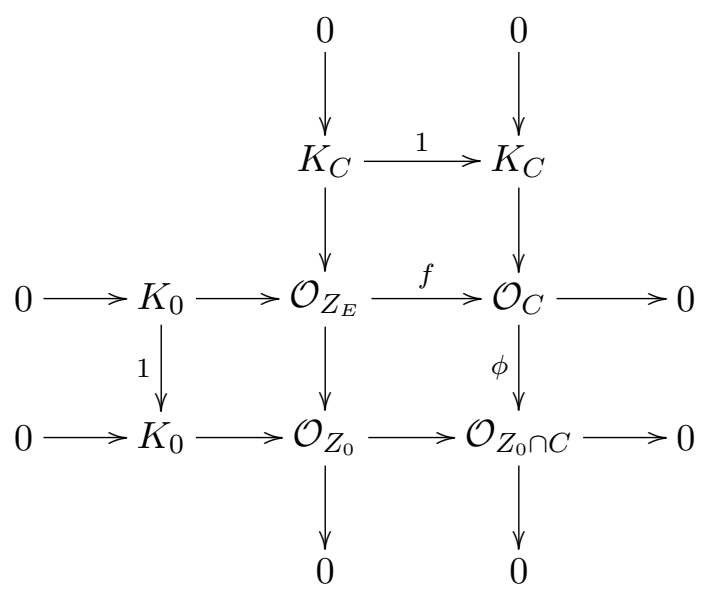

where $Z_{0} \subset X$ is a pure dimension one closed subscheme of $X$ with topological support on $C_{0}$, and $\mathcal{O}_{Z_{0} \cap C}$ the structure sheaf of the scheme theoretic intersection $Z_{0} \cap C$ in $X$.

(iii) $\chi\left(K_{0}\right) \geq 0$.

Proof. Purity of $\mathcal{O}_{Z_{E}}$ follows from the observation that any nontrivial morphism $F \rightarrow \mathcal{O}_{Z_{E}}$ with $F$ zero-dimensional would yield via the exact sequences (2.7), (2.8) a nontrivial morphism $F \rightarrow E$ in $\mathcal{A}^{C}$. This contradicts the assumption that $E$ belongs to $\mathcal{A}_{1 / 2}^{C}$.

Next, the given conditions on the Chern classes of $E$ imply that

$$
\operatorname{ch}_{2}\left(\mathcal{H}^{0}(E)\right)=r_{0}\left[C_{0}\right], \quad \operatorname{ch}_{2}\left(\mathcal{H}^{-1}(E)\right)=-[C]-r_{-1}\left[C_{0}\right]
$$

with $r_{0}, r_{-1} \geq 0, r_{0}+r_{-1}=r$. Moreover, there is an exact sequence of $\mathcal{O}_{X^{-}}$ modules

$$
0 \rightarrow K_{0} \rightarrow \mathcal{O}_{Z_{E}} \rightarrow \mathcal{O}_{C} \rightarrow 0
$$

where $K_{0}$ is a pure dimension one sheaf with topological support on $C_{0}$. According to Lemma 2.6, there is an isomorphism

$$
\varphi_{1}: \operatorname{Ext}_{X}^{1}\left(\mathcal{O}_{C}, K_{0}\right) \simeq \operatorname{Hom}_{X}\left(\mathcal{O}_{C}, \mathcal{O}_{D} \otimes_{X} K_{0}\right)
$$

identifying the extension class $e \in \operatorname{Ext}_{X}^{1}\left(\mathcal{O}_{C}, K_{0}\right)$ determined by (2.11) with a morphism $\phi \in \operatorname{Hom}_{X}\left(\mathcal{O}_{C}, \mathcal{O}_{D} \otimes_{X} K_{0}\right)$. Let $K_{C}=\operatorname{Ker}(\phi)$ and $I=\operatorname{Im}(\phi)$. 
Then Lemma 2.6 also implies that the restriction of the extension class $e$ to $K_{C} \subset \mathcal{O}_{C}$ is trivial. Therefore there is a commutative diagram

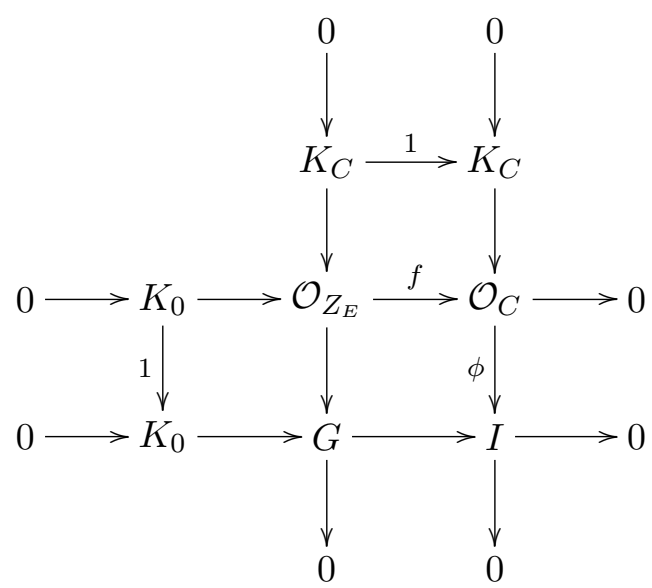

with exact rows and columns. Obviously, $G$ is the structure sheaf of a closed subscheme $Z_{0} \subset X$. The support conditions on $\mathcal{O}_{Z_{E}}$ and Equations (2.10) imply that $G$ is topologically supported on $C_{0}$ and $\operatorname{ch}_{2}(G)=r_{-1}\left[C_{0}\right]$. Moreover $I$ is isomorphic to the structure sheaf $\mathcal{O}_{Z_{0} \cap C}$ of the scheme theoretic intersection $Z_{0} \cap C$.

In order to prove that $G$ is pure, suppose $T \subset G$ is the maximal zerodimensional subsheaf and let $G^{\prime}=G / T$. Then $G^{\prime}$ is pure dimension one with $\operatorname{ch}_{2}\left(G^{\prime}\right)=r_{-1}\left[C_{0}\right]$. Let $K_{C}^{\prime}$ be the kernel of the resulting epimorphism $\mathcal{O}_{Z_{E}} \rightarrow G^{\prime}$. Then there is a commutative diagram

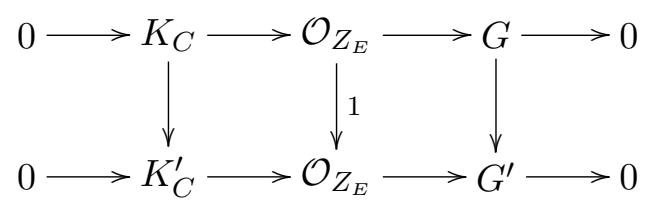

which implies that the morphism $K_{C} \rightarrow K_{C}^{\prime}$ is injective and $K_{C}^{\prime} / K_{C} \simeq T$. Hence $K_{C}^{\prime}$ is a pure dimension one subsheaf of $\mathcal{O}_{Z_{E}}$ with support on $C$ and $\operatorname{ch}_{2}\left(K_{C}^{\prime}\right)=\operatorname{ch}_{2}\left(K_{C}\right)=[C]$. Then the restriction $\left.f\right|_{K_{C}^{\prime}}: K_{C}^{\prime} \rightarrow \mathcal{O}_{C}$ must be injective since $\operatorname{Hom}_{X}\left(K_{C}^{\prime}, K_{0}\right)=0$. Therefore, the restriction

$$
0 \rightarrow K_{0} \rightarrow F \rightarrow K_{C}^{\prime} \rightarrow 0
$$

of the extension (2.11) to $K_{C}^{\prime} \subset \mathcal{O}_{C}$ must be trivial. This implies that $K_{C}^{\prime}$ is contained in the kernel of $\phi$, which is $K_{C}$. Therefore, $K_{C}^{\prime}=K_{C}$, which implies $G^{\prime}=G$, and $T=0$. In conclusion, $G$ is of pure dimension one. 
The third statement of Lemma 2.8 follows from the observation that the canonical surjective morphism $\mathcal{O}_{X} \rightarrow \mathcal{O}_{Z_{0}}$ determines a stable pair on $X$ with support on $C_{0}$. According to Lemma 2.5, this implies that

$$
\chi\left(\mathcal{O}_{Z_{0}}\right) \geq r_{-1}
$$

However, since $K_{0}$ is pure dimension one with topological support on $C_{0}$, and the divisor $D$ is transverse to $C_{0}$, there is an exact sequence

$$
0 \rightarrow K_{0}(-D) \rightarrow K_{0} \rightarrow \mathcal{O}_{D} \otimes_{X} K_{0} \rightarrow 0
$$

Then the Riemann-Roch theorem yields

$$
\chi\left(\mathcal{O}_{D} \otimes_{X} K_{0}\right)=r_{-1}
$$

as $\operatorname{ch}_{2}\left(K_{0}\right)=r_{-1}\left[C_{0}\right]$ and $C_{0} \cdot D=1$. Since $\mathcal{O}_{Z_{0} \cap C} \subseteq \mathcal{O}_{D} \otimes_{X} K_{0}$ is an inclusion of zero-dimensional sheaves, $\chi\left(\mathcal{O}_{Z_{0} \cap C}\right) \leq r_{-1}$. Therefore

$$
\chi\left(K_{0}\right)=\chi\left(\mathcal{O}_{Z_{0}}\right)-\chi\left(\mathcal{O}_{Z_{0} \cap C}\right) \geq 0
$$

Next note that there is an injective morphism

$$
\mathcal{O}_{Z_{E}} \hookrightarrow \mathcal{H}^{-1}(E)[1]
$$

in $\mathcal{A}^{C}$ corresponding to the canonical extension

$$
0 \rightarrow \mathcal{H}^{-1}(E) \rightarrow \mathcal{O}_{X} \rightarrow \mathcal{O}_{Z_{E}} \rightarrow 0
$$

Therefore, the canonical inclusion $K_{0} \subset \mathcal{O}_{Z_{E}}$ is a subobject of $\mathcal{H}^{-1}(E)[1] \subset E$.

Lemma 2.9. Suppose $E$ is an object of $\mathcal{A}_{1 / 2}^{C}$ with $\operatorname{ch}_{0}(E)=-1, \operatorname{ch}_{2}(E)=$ $[C]+r\left[C_{0}\right], r \geq 0$. Then there is an exact sequence

$$
0 \rightarrow K_{0} \rightarrow E \rightarrow G \rightarrow 0
$$

in $\mathcal{A}^{C}$ where $\mathcal{H}^{-1}(G) \simeq \mathcal{I}_{C}$ and $\mathcal{H}^{0}(G) \simeq \mathcal{H}^{0}(E)$. 
Proof. Since $K_{0}$ is pure of dimension one, it belongs to $\mathcal{A}_{1 / 2}^{C}$. According to Lemma 2.2, $G=E / K_{0}$ belongs to $\mathcal{A}^{C}$. Moreover, note that the morphism

$$
K_{0} \rightarrow E \rightarrow \mathcal{H}^{0}(E)
$$

is trivial since $K_{0}$ is a subobject of $\mathcal{H}^{-1}(E)[1]$. Then the long exact cohomology sequence of (2.13) yields exact sequences of $\mathcal{O}_{X}$-modules

$$
\begin{aligned}
0 & \rightarrow \mathcal{H}^{-1}(E) \rightarrow \mathcal{H}^{-1}(G) \rightarrow K_{0} \rightarrow 0 \\
0 & \rightarrow \mathcal{H}^{0}(E) \rightarrow \mathcal{H}^{0}(G) \rightarrow 0
\end{aligned}
$$

The first exact sequence is the restriction of (2.12) to $K_{0} \subset \mathcal{O}_{Z_{E}}$. Then a simple application of the snake lemma shows that $\mathcal{H}^{-1}(G) \simeq \mathcal{I}_{C}$.

Finally note the following observation.

Lemma 2.10. Let $E$ be an object of $\mathcal{A}_{1 / 2}^{C}$ with $\operatorname{ch}(E)=(-1,0,[C], n), n \in$ $\mathbb{Z}$ such that $\mathcal{H}^{0}(E)$ is a zero-dimensional sheaf. Then $E$ is isomorphic to a stable pair $P_{C}=\left(\mathcal{O}_{X} \stackrel{s}{\longrightarrow} F_{C}\right)$ with $F_{C}$ a pure dimension one sheaf with scheme theoretic support on $C$. Moreover $E$ is $\mu_{(\omega, b)}$-stable for any $b \in \mathbb{R}$.

Proof. The first part follows from [51, Lemm. 4.5]. In particular, there is an exact sequence

$$
0 \rightarrow F_{C} \rightarrow E \rightarrow \mathcal{O}_{X}[1] \rightarrow 0
$$

in $\mathcal{A}$. For the second, note that there are no strict epimorphisms $E \rightarrow G$ in $\mathcal{A}_{1 / 2}^{C}$ with $G$ pure dimension one since $\operatorname{Hom}_{\mathcal{A}}(E, G) \subset \operatorname{Hom}_{X}\left(\mathcal{H}^{0}(E), G\right)$ and $\mathcal{H}^{0}(E)$ is zero-dimensional. Furthermore, for any pure dimension one sheaf $G$ with support on $C_{0}$, there is an exact sequence

$$
0 \rightarrow \operatorname{Hom}_{\mathcal{A}}(E, G) \rightarrow \operatorname{Ext}_{X}^{1}\left(\mathcal{O}_{X}, G\right) \rightarrow \cdots
$$

since $\operatorname{Hom}_{X}\left(G, F_{C}\right)=0$. Serre duality yields and isomorphism $\operatorname{Ext}_{X}^{1}$ $\left(\mathcal{O}_{X}, G\right) \simeq H^{2}(G)^{\vee}$, hence there are no strict monomorphisms $G \hookrightarrow E$ in $\mathcal{A}_{1 / 2}^{C}$.

\section{Stable pairs at small $b$}

The goal of this section is to analyze the structure of the moduli stacks $\mathcal{P}_{(\omega, b)}(X, C, r, n)$ for $b>0$ sufficiently close to 0 , in particular to prove Theorem 1.3. Here again $X=X^{+}$and $C=C^{+}$and one fixes a Kähler class 
$\omega$ such that $\int_{C_{0}} \omega=1$. For the present purposes it suffices to consider only generic values of $b$, in which case strictly semistable objects with numerical invariants $(r, n)$ do not exist. The main technical result in this section is the stability criterion obtained in Proposition 3.3 below. The proof is fairly long and complicated, and will be carried out in several stages in Section 3.1. Sections 3.2 and 3.3 summarize several applications of this result, explaining the connection between moduli stacks of $C$-framed perverse coherent sheaves and nested Hilbert schemes.

\subsection{A stability criterion}

Fix $\operatorname{ch}(E)=\left(-1,0,[C]+r\left[C_{0}\right], n\right), r \in \mathbb{Z} \geq 0, n \in \mathbb{Z}$ and Kähler class $\omega$ on $X$ such that $\int_{C_{0}} \omega=1$.

Lemma 3.1. For fixed Kähler class $\omega$, and fixed $(r, n) \in \mathbb{Z}_{\geq 0} \times \mathbb{Z}$ the following holds for any stability parameter $b>0$ such that

$$
b<\frac{1}{2 r}
$$

if $r>0$.

Any $\mu_{(\omega, b)}$-stable object $E$ of $\mathcal{A}^{C}$ with $\operatorname{ch}(E)=\left(-1,0,[C]+r\left[C_{0}\right], n\right)$ fits in an exact sequence

$$
0 \rightarrow P_{C} \rightarrow E \rightarrow G \rightarrow 0
$$

in $\mathcal{A}^{C}$ such that

(i) $P_{C}=\left(\mathcal{O}_{X} \stackrel{s}{\longrightarrow} F_{C}\right)$ is a stable pair on $X$ with $F_{C}$ scheme theoretically supported on $C$.

(ii) $G$ is a pure dimension one sheaf on $X$ with topological support on $C_{0}$ and $\operatorname{ch}_{2}(G)=r\left[C_{0}\right]$. Moreover its Harder-Narasimhan filtration

$$
0=G_{0} \subset G_{1} \subset \cdots \subset G_{h}=G
$$

with respect to $\omega$-slope stability satisfies

$$
G_{j} / G_{j-1} \simeq \mathcal{O}_{C_{0}}\left(a_{j}\right)^{\oplus s_{j}}
$$

where $a_{j} \in \mathbb{Z}_{\geq-1}$ for $j=1, \ldots, h$, and $a_{1}>a_{2}>\cdots>a_{h}$. 
Proof. It will be first proven that for $b>0$ sufficiently close to 0 , the stability criterion in Proposition 2.1 implies that $\mathcal{H}^{-1}(E)=\mathcal{I}_{C}$ for any $\mu_{(\omega, b)}$-stable object $E$ of $\mathcal{A}^{C}$ with $\operatorname{ch}(E)=\left(-1,0,[C]+r\left[C_{0}\right], n\right)$.

Suppose $E$ is $\mu_{(\omega, b)}$-stable for some $b>0$. This implies that $E$ is an element of $\mathcal{A}_{1 / 2}^{C}$ satisfying the stability criterion in Proposition 2.1 with respect to strict morphisms in $\mathcal{A}_{1 / 2}^{C}$. According to Lemmas 2.9, 2.8 there is an injective morphism $\kappa: K_{0} \rightarrow E$ where $K_{0}$ is pure of dimension one and $\chi\left(K_{0}\right) \geq 0$. Suppose $K_{0}$ is nontrivial, and $\operatorname{ch}_{2}\left(K_{0}\right)=r_{-1}\left[C_{0}\right]$ for some $r_{-1}>0$. Lemma 2.3 implies that the cokernel $G=\operatorname{Coker}(\kappa)$ fits in an exact sequence

$$
0 \rightarrow G_{1} \rightarrow G \rightarrow G_{1 / 2} \rightarrow 0
$$

where $G_{i} \in \mathcal{A}_{i}^{C}, i=1,1 / 2$. In particular, $G_{1}$ is a zero-dimensional sheaf. Then the snake lemma implies that the kernel $K$ of the projection $E \rightarrow G_{1 / 2}$ is a $1 \mathrm{D}$ sheaf on $X$ which fits in an exact sequence

$$
0 \rightarrow K_{0} \rightarrow K \rightarrow G_{1} \rightarrow 0
$$

Moreover, $K$ must be pure of dimension one since $E$ belongs to $\mathcal{A}_{1 / 2}^{C}$. Then the stability criterion in Proposition 2.1 implies that

$$
\frac{\chi(K)}{r_{-1}}<-2 b
$$

if $r_{-1}>0$. Since $b>0$, this implies that $\chi(K)<0$, hence also $\chi\left(K_{0}\right)<0$ since $G_{1}$ is a zero-dimensional sheaf. This contradicts Lemma $2.8(\mathrm{iii})$ unless $K_{0}$ is trivial. In conclusion, $\mathcal{H}^{-1}(E)=\mathcal{I}_{C}$ for any $b>0$.

This implies in particular that $\operatorname{ch}_{2}\left(\mathcal{H}^{0}(E)\right)=r\left[C_{0}\right]$ for $b>0$. Therefore, if $r=0, \mathcal{H}^{0}(E)$ must be a zero-dimensional sheaf with topological support on $C$. Since $E$ belongs to $\mathcal{A}_{1 / 2}^{C}$, and $\operatorname{ch}_{2}(E)=[C]$, Lemma 2.10 implies that $E$ must be isomorphic to a stable pair $P_{C}=\left(\mathcal{O}_{X} \stackrel{s}{\longrightarrow} F_{C}\right)$, with $F_{C}$ scheme theoretically supported on $C$.

Next suppose $r>0, E$ is $\mu_{(\omega, b)}$-stable for some $b>0$, and let $\mathcal{H}^{0}(E) \rightarrow$ $G$ be a nontrivial pure dimension one quotient of $\mathcal{H}^{0}(E)$. Condition (C.2) implies that $G$ is topologically supported on $C_{0}$, and $\operatorname{ch}_{2}(G)=r_{G}\left[C_{0}\right]$ for some $0<r_{G} \leq r$. Let $F$ be the kernel of the epimorphism $E \rightarrow G$. Then $F$ belongs to $\mathcal{A}_{1 / 2}^{C}$ since $E$ does, hence the epimorphism $E \rightarrow G$ is strict. Moreover $\mathcal{H}^{-1}(F) \simeq \mathcal{H}^{-1}(E)$. Then the stability criterion in Proposition 2.1 
implies that

$$
\frac{\chi(G)}{r_{G}}>-2 b .
$$

Note that $-2 b>-1 / r$ if the bound (3.1) holds. Since $0<r_{G} \leq r$ this implies that

$$
\chi(G)>-\frac{r_{G}}{r} \geq-1
$$

hence $\chi(G) \geq 0$, since $\chi(G) \in \mathbb{Z}$.

Now consider the exact sequence

$$
0 \rightarrow Q \rightarrow \mathcal{H}^{0}(E) \rightarrow G \rightarrow 0
$$

where $Q \subset \mathcal{H}^{0}(E)$ is the maximal zero-dimensional subsheaf of $\mathcal{H}^{0}(E)$ and $G$ is pure of dimension one supported on $C_{0}$. The previous argument implies that any pure dimension one quotient $G \rightarrow G^{\prime}$ must have $\chi\left(G^{\prime}\right) \geq 0$ if (3.1) holds. In particular, using Lemma 2.4, $G$ has a Harder-Narasimhan filtration of the form (3.3).

Let $E^{\prime}=\operatorname{Ker}(E \rightarrow G)$. Obviously, $E^{\prime}$ belongs to $\mathcal{A}_{1 / 2}^{C}$ and there is an exact sequence

$$
0 \rightarrow \mathcal{I}_{C}[1] \rightarrow E^{\prime} \rightarrow Q \rightarrow 0
$$

in $\mathcal{A}^{C}$. Then [51, Lemm. 4.5] implies that $E^{\prime}$ is isomorphic to the stable pair $P_{C}=\left(\mathcal{O}_{X} \stackrel{s}{\longrightarrow} F_{C}\right)$ in $\mathcal{A}^{C}$, where $s$ is determined by the natural projection $\mathcal{O}_{X} \rightarrow \mathcal{O}_{C}$.

Lemma 3.2. Under the same conditions as in Lemma 3.1 suppose the bound (3.1) is satisfied. Then for any $\mu_{(\omega, b)}$-stable object $E$ of $\mathcal{A}^{C}$ with $\operatorname{ch}(E)=\left(-1,0,[C]+r\left[C_{0}\right], n\right)$, with $r>0$, the quotient $G$ in $(3.2)$ must be of the form $G \simeq \mathcal{O}_{C_{0}}(-1)^{\oplus r}$.

Proof. Using the notation in Lemma 3.1 note the exact sequence

$$
0 \rightarrow F_{C} \rightarrow P_{C} \rightarrow \mathcal{O}_{X}[1] \rightarrow 0
$$

in $\mathcal{A}$, which yields a long exact sequence exact sequence

$$
\cdots \rightarrow \operatorname{Ext}_{X}^{1}\left(G, \mathcal{O}_{X}\right) \rightarrow \operatorname{Ext}_{X}^{1}\left(G, F_{C}\right) \rightarrow \operatorname{Ext}_{\mathcal{A}}^{1}\left(G, P_{C}\right) \rightarrow \operatorname{Ext}_{X}^{2}\left(G, \mathcal{O}_{X}\right) \cdots
$$

Note also that

$$
\operatorname{Ext}_{X}^{1}\left(G, \mathcal{O}_{X}\right) \simeq H^{2}(X, G)^{\vee}=0
$$


by Serre duality and the structure of the Harder-Narasimhan filtration of $G$ implies

$$
\operatorname{Ext}^{2}\left(G, \mathcal{O}_{X}\right) \simeq H^{1}(X, G)^{\vee}=0
$$

as well. Therefore, there is an isomorphism

$$
\operatorname{Ext}_{X}^{1}\left(G, F_{C}\right) \simeq \operatorname{Ext}_{\mathcal{A}}^{1}\left(G, P_{C}\right)
$$

This implies that there is an extension

$$
0 \rightarrow F_{C} \rightarrow F \rightarrow G \rightarrow 0
$$

determined by the extension class of $E$ up to isomorphism, which fits in a commutative diagram

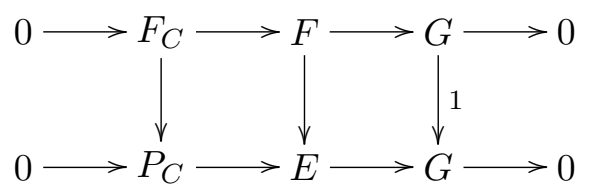

in $\mathcal{A}$. In particular, the middle vertical morphism is injective in $\mathcal{A}$, and

$$
E / F \simeq P_{C} / F_{C} \simeq \mathcal{O}_{X}[1]
$$

Since $E$ belongs to $\mathcal{A}_{1 / 2}^{C}, F$ has to be a pure dimension one sheaf.

Let $F_{C}^{\prime}$ be the quotient $F \otimes_{X} \mathcal{O}_{C} / T$ where $T \subset F \otimes_{X} \mathcal{O}_{C}$ is the maximal zero-dimensional submodule. Then there is an exact sequence

$$
0 \rightarrow G^{\prime} \rightarrow F \rightarrow F_{C}^{\prime} \rightarrow 0
$$

where $G^{\prime}$ is pure dimension one with topological support of $C_{0}$ and $F_{C}^{\prime}$ pure dimension one with support on $C$. Moreover, $\operatorname{ch}_{2}\left(G^{\prime}\right)=\operatorname{ch}_{2}(G)=r\left[C_{0}\right]$ and $\operatorname{ch}_{2}\left(F_{C}^{\prime}\right)=\operatorname{ch}_{2}\left(F_{C}\right)=[C]$. In particular, $F_{C}^{\prime}$ is scheme theoretically supported on $C$. Obviously $G^{\prime} \subset F \subset E$ in $\mathcal{A}^{C}$, and there is an exact sequence

$$
0 \rightarrow F_{C}^{\prime} \rightarrow E / G^{\prime} \rightarrow \mathcal{O}_{X}[1] \rightarrow 0
$$

in $\mathcal{A}$. This implies that $E / G^{\prime}$ belongs to $\mathcal{A}_{1 / 2}$ since both $F_{C}^{\prime}, \mathcal{O}_{X}[1]$ do. As $E / G^{\prime}$ also belongs to $\mathcal{A}^{C}$ according to Lemma 2.2 , it follows that $E / G^{\prime}$ belongs to $\mathcal{A}_{1 / 2}^{C}$, i.e., the inclusion $G^{\prime} \subset E$ is strict. 
The same holds for any proper saturated subsheaf $G^{\prime \prime} \subset G^{\prime}$ (that is a subsheaf such that $G^{\prime} / G^{\prime \prime}$ is pure dimension one). For any such sheaf, there is an exact sequence

$$
0 \rightarrow G^{\prime} / G^{\prime \prime} \rightarrow E / G^{\prime \prime} \rightarrow E / G^{\prime} \rightarrow 0
$$

in $\mathcal{A}$ which implies that $E / G^{\prime \prime}$ belongs to $\mathcal{A}_{C}^{1 / 2}$. Then the stability criterion implies that

$$
\frac{\chi\left(G^{\prime \prime}\right)}{r_{G^{\prime \prime}}}<-2 b<0
$$

for any saturated subsheaf $G^{\prime \prime} \subseteq G^{\prime}$, where $\operatorname{ch}_{2}\left(G^{\prime \prime}\right)=r_{G^{\prime \prime}}\left[C_{0}\right], 0<r_{G^{\prime \prime}} \leq r$.

Next recall that according to Lemma 2.6 there is an isomorphism

$$
\varphi_{1}: \operatorname{Ext}_{X}^{1}\left(F_{C}^{\prime}, G^{\prime}\right) \stackrel{\sim}{\longrightarrow} \operatorname{Hom}_{D}\left(F_{C}^{\prime}, \mathcal{O}_{D} \otimes_{X} G^{\prime}\right)
$$

Since $G^{\prime}$ is pure dimension one supported on $C_{0}$, there is an exact sequence

$$
0 \rightarrow G^{\prime}(-D) \rightarrow G^{\prime} \rightarrow \mathcal{O}_{D} \otimes_{X} G^{\prime} \rightarrow 0
$$

This implies via the Riemann-Roch theorem that

$$
\chi\left(\mathcal{O}_{D} \otimes_{X} G^{\prime}\right)=r
$$

Now let $\phi: F_{C}^{\prime} \rightarrow \mathcal{E} x t_{D}^{0}\left(\mathcal{O}_{D}, G^{\prime}\right)$ be the morphism corresponding to the extension class of (3.9) under the isomorphism (3.11). The exact sequences (3.7), (3.9) imply that there is an injective morphism $F_{C} \hookrightarrow F_{C}^{\prime}$ such that the following diagram commutes:

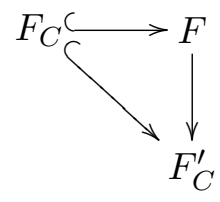

since both $F_{C}, F_{C}^{\prime}$ are pure supported on $C$ and $\operatorname{ch}_{2}\left(F_{C}\right)=\operatorname{ch}_{2}\left(F_{C}^{\prime}\right)=[C]$. This implies that the restriction of the extension (3.9) to $F_{C} \subset F_{C}^{\prime}$ is split i.e., $F_{C} \subset \operatorname{Ker}(\phi)$. In conclusion, the quotient $F_{C}^{\prime} / F_{C}$ is a subsheaf of $\mathcal{O}_{D} \otimes_{X} G^{\prime}$ 
and there is a commutative diagram

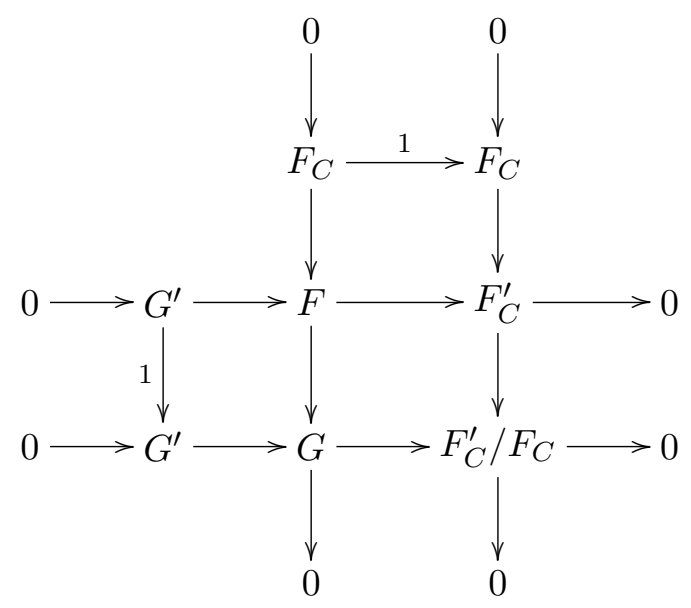

with exact rows and columns. The bottom row of this diagram yields

$$
\chi\left(G^{\prime}\right)=\chi(G)-\chi\left(F_{C}^{\prime} / F_{C}\right) \geq \chi(G)-\chi\left(\mathcal{O}_{D} \otimes_{X} G^{\prime}\right)=\chi(G)-r
$$

In order to conclude the proof, let

$$
0=G_{0}^{\prime} \subset G_{i}^{\prime} \subset \cdots \subset G_{h^{\prime}}^{\prime}=G
$$

be the Harder-Narasimhan filtration of $G^{\prime}$ with respect to $\omega$-slope stability. Each nontrivial quotient $G_{j}^{\prime} / G_{j-1}^{\prime}, j=1, \ldots, h^{\prime}$ must be isomorphic to a sheaf of the form $\mathcal{O}_{C_{0}}\left(a_{j}^{\prime}\right)^{s_{j}^{\prime}}, s_{j}^{\prime} \geq 1$, such that $a_{1}^{\prime}>a_{2}^{\prime}>\cdots>a_{h^{\prime}}^{\prime}$ by Lemma 2.4. Inequality (3.10) implies that $a_{1}^{\prime} \leq-2$, therefore $a_{j}^{\prime} \leq-2$ for all $j=1, \ldots, h^{\prime}$. This implies

$$
\chi\left(G^{\prime}\right) \leq-r
$$

hence inequality (3.12) yields

$$
\chi(G) \leq 0
$$

Taking into account the constraints on the Harder-Narasimhan filtration of $G$ in Lemma 3.1 it follows that

$$
G \simeq \mathcal{O}_{C_{0}}(-1)^{\oplus r}
$$


Proposition 3.3. For fixed Kähler class $\omega$, and fixed $(r, n) \in \mathbb{Z}_{\geq 0} \times \mathbb{Z}$ the following holds for any $b>0$ satisfying the bound (3.1).

An object $E$ of $\mathcal{A}^{C}$ is $\mu_{(\omega, b)}$-stable if and only if there is an exact sequence of the form

$$
0 \rightarrow P_{C} \rightarrow E \rightarrow \mathcal{O}_{C_{0}}(-1)^{\oplus r} \rightarrow 0
$$

in $\mathcal{A}^{C}$ such that:

(i) $P_{C}=\left(\mathcal{O}_{X} \rightarrow F_{C}\right)$ is a stable pair on $X$ with $F_{C}$ scheme theoretically supported on $C$.

(ii) There is no linear subspace $0 \subset V^{\prime} \subset \mathbb{C}^{r}$ such that the restriction

$$
0 \rightarrow P_{C} \rightarrow E^{\prime} \rightarrow V^{\prime} \otimes \mathcal{O}_{C_{0}}(-1) \rightarrow 0
$$

of the extension (3.13) to $V^{\prime} \otimes \mathcal{O}_{C_{0}}(-1)$ is trivial. Extensions (3.13) satisfying this property will be called nondegenerate.

Remark 3.4. Since $\operatorname{Ext}_{X}^{2}\left(\mathcal{O}_{C_{0}}(-1)^{\oplus r}, \mathcal{O}_{X}\right)=0$, there is an isomorphism

$$
\operatorname{Ext}_{X}^{1}\left(\mathcal{O}_{C_{0}}(-1)^{\oplus r}, P_{C}\right) \simeq \operatorname{Ext}_{X}^{1}\left(\mathcal{O}_{C_{0}}(-1)^{\oplus r}, F_{C}\right)
$$

for any stable pair $P_{C}=\left(\mathcal{O}_{X} \rightarrow F_{C}\right)$. This observation will be used in the proof of Proposition 3.11.

Proof. $(\Rightarrow)$ First note that if $r=0$ Lemma 3.1 shows that $E$ must be isomorphic to a stable pair $P_{C}$. Furthermore, any such stable pair is stable for all $b>0$ according to Lemma 2.10 .

Suppose $r>0$. The existence of an extension of the form (3.13) follows from Lemmas 3.1 and 3.2. Nondegeneracy follows easily noting that if the restriction of the extension (3.13) to some subsheaf $G^{\prime}=V^{\prime} \otimes \mathcal{O}_{C_{0}}(-1) \subset$ $\mathcal{O}_{C_{0}}(-1)^{\oplus r}$ is trivial, then there is an epimorphism $G^{\prime} \hookrightarrow E$. Moreover, there is an exact sequence

$$
0 \rightarrow P_{C} \rightarrow E / G^{\prime} \rightarrow V^{\prime \prime} \otimes \mathcal{O}_{C_{0}}(-1) \rightarrow 0
$$

in $\mathcal{A}$ where $V^{\prime \prime} \simeq \mathbb{C}^{r} / V^{\prime}$. This implies that that $E / G^{\prime}$ belongs to $\mathcal{A}_{1 / 2}^{C}$ i.e., the epimorphism $G^{\prime} \hookrightarrow E$ is strict. Then $G^{\prime} \subset E$ violates the stability criterion for $b>0$.

$(\Leftarrow)$ Conversely, suppose an object $E$ of $\mathcal{A}^{C}$ fits in an extension of the form (3.2) satisfying the nondegeneracy condition. Then it follows easily that 
$E$ belongs to $\mathcal{A}_{1 / 2}^{C}$. One has to check the stability criterion in Proposition 2.1 with respect to strict monomorphisms and epimorphisms in $\mathcal{A}^{C}$. Note that property (C.2) implies that all pure dimension one sheaves in $\mathcal{A}^{C}$ must be topologically supported on $C_{0}$.

Consider first strict epimorphisms $E \rightarrow G$, with $G$ a pure dimension one sheaf supported on $C_{0}$. It is straightforward to check that $\operatorname{Hom}_{\mathcal{A}}\left(P_{C}, G\right)=0$ as in the proof of Lemma 2.10. Therefore the exact sequence (3.13) yields an isomorphism

$$
\operatorname{Hom}_{\mathcal{A}}(E, G) \simeq \operatorname{Hom}_{X}\left(\mathcal{O}_{C_{0}}(-1)^{\oplus r}, G\right) .
$$

Since $\mathcal{O}_{C_{0}}(-1)^{\oplus r}$ is $\omega$-slope semistable, any quotient $G$ must satisfy

$$
\chi(G) \geq 0>-2 b
$$

for any $b>0$.

Next suppose $F \hookrightarrow E$ is a strict monomorphism in $\mathcal{A}_{C}$ with $F$ a nontrivial pure dimension one sheaf on $X$ supported on $C_{0}$. Let $F^{\prime}$ denote the image of $F$ in $\mathcal{O}_{C_{0}}(-1)^{\oplus r}$ and $F^{\prime \prime}$ the kernel of $F \rightarrow F^{\prime}$. Then $F^{\prime \prime}$ must be a subobject of $P_{C}$ in $\mathcal{A}^{C}$, hence it must be trivial, as shown in the proof of Lemma 2.10. Therefore $F=F^{\prime}$ must be a subsheaf of $\mathcal{O}_{C_{0}}(-1)^{\oplus r}$, which is $\omega$-slope semistable with $\chi\left(\mathcal{O}_{C_{0}}(-1)\right)=0$. This implies $\chi(F) \leq 0$. Since the bound (3.1) yields,

$$
-\frac{1}{r}<-2 b<0
$$

$F$ destabilizes $E$ only if $\chi(F)=0$, which implies that $F \simeq V^{\prime} \otimes \mathcal{O}_{C_{0}}(-1)$ for some linear subspace $V^{\prime} \subseteq \mathbb{C}^{r}$. However this contradicts the nondegeneracy assumption.

\subsection{Moduli spaces of decorated sheaves}

Consider the moduli problem for data $(V, L, F, s, f)$ where $V, L$ are vector spaces of dimension $r, 1, r \geq 1$, respectively, $F$ is a coherent sheaf on $X$, and

$$
s: L \otimes \mathcal{O}_{X} \rightarrow F, \quad f: F \rightarrow V \otimes \mathcal{O}_{C_{0}}(-1)
$$

are morphisms of coherent sheaves satisfying the following conditions:

(a) $F$ is pure of dimension one with $\operatorname{ch}_{2}(F)=[C]+r\left[C_{0}\right], \chi(F)=n$.

(b) $f: F \rightarrow V \otimes \mathcal{O}_{C_{0}}(-1)$ is surjective and $\operatorname{Ker}(f)$ is scheme theoretically supported on $C$. 
(c) $s: L \otimes \mathcal{O}_{X} \rightarrow F$ is a nonzero morphism.

(d) The extension

$$
0 \rightarrow \operatorname{Ker}(f) \rightarrow F \rightarrow V \otimes \mathcal{O}_{C_{0}}(-1) \rightarrow 0
$$

satisfies the nondegeneracy condition of Proposition 3.3. That is there is no proper nontrivial subspace $0 \subset V^{\prime} \subset V$ such that the restriction of the above extension to $V^{\prime} \otimes \mathcal{O}_{C_{0}}(-1)$ is trivial.

Two collections $(V, L, F, s, f),\left(V^{\prime}, L^{\prime}, F^{\prime}, s^{\prime}, f^{\prime}\right)$ are isomorphic if there exist linear isomorphisms $V \stackrel{\sim}{\longrightarrow} V^{\prime}, L \stackrel{\sim}{\longrightarrow} L^{\prime}$ and an isomorphism of sheaves $F \stackrel{\sim}{\longrightarrow} F^{\prime}$ satisfying the obvious compatibility conditions with the data $(s, f)$, $\left(s^{\prime}, f^{\prime}\right)$ are satisfied. Then it is straightforward to prove the automorphism group of any collection $(V, L, F, s, f)$ is isomorphic to $\mathbb{C}^{\times}$.

Let $T$ be a scheme over $\mathbb{C}, X_{T}=X \times T$ and $\pi_{T}: X_{T} \rightarrow T$ denote the canonical projection. For any closed point $t \in T$, let $X_{t}=X \times\{t\}$ denote the fiber of $\pi_{T}$ over $t$. Let also $C_{0 T} \subset X_{T}, C_{T} \subset X_{T}$ denote the closed subschemes $C_{0} \times T \subset X \times T, C \times T \subset X \times T$ respectively, and $\mathcal{O}_{C_{0 T}}(d)$ denote the pull-back of the sheaf $\mathcal{O}_{C_{0}}(d)$ to $X_{T}$, for any $d \in \mathbb{Z}$. Similar notation will be employed for each closed fiber $X_{t}, t \in T$.

A flat family of data $(V, L, F, s, f)$ on $X$ parameterized by $T$ is a collection $\left(V_{T}, L_{T}, F_{T}, s_{T}, f_{T}\right)$ where

- $V_{T}, L_{T}$ are locally free $\mathcal{O}_{T}$-modules and $F_{T}$ is a coherent $\mathcal{O}_{X_{T}}$-module flat over $T$.

- $s_{T}: \pi_{T}^{*} V_{T} \rightarrow F_{T}$ and $f_{T}: F_{T} \rightarrow \pi_{T}^{*} L_{T} \otimes_{X_{T}} \mathcal{O}_{C_{0 T}}$ are morphisms of $\mathcal{O}_{X_{T}}$-modules

- The restriction of the data $\left(V_{T}, L_{T}, F_{T}, s_{T}, f_{T}\right)$ to any fiber $X_{t}$, with $t \in$ $T$, a closed point is a collection satisfying conditions $(a)-(d)$ above.

For any $(r, n) \in \mathbb{Z}_{\geq 0} \times \mathbb{Z}$ let $\mathcal{Q}(X, C, r, n)$ denote the resulting moduli stack of data $(V, L, F, s, f)$ on $X$ satisfying conditions $(a)-(d)$. Let $Q(X, C$, $r, n)$ be the rigidification of $\mathcal{Q}(X, C, r, n)$ obtained by fixing isomorphisms $L \simeq \mathbb{C}$ and $V \simeq \mathbb{C}^{r}$. Then the closed points of $Q(X, C, r, n)$ have trivial stabilizers and $\mathcal{Q}(X, C, r, n)$ is a $\mathbb{C}^{\times}$-gerbe over $Q(X, C, r, n)$.

The moduli stacks $\mathcal{Q}(X, C, r, n)$ will be used as an interpolating tool between the nested Hilbert schemes $H_{p}^{[n, k]}(C)$ introduced in Section 1.1.1 and stable $C$-framed perverse coherent sheaves at small $b>0$. 
Remark 3.5. For future reference, let $\mathcal{M}(X, C, r, n)$ denote the moduli stack of data $(V, f, F)$ satisfying conditions $(a),(b),(d)$ above. Obviously, there is a natural morphism $\pi: \mathcal{Q}(X, C, r, n) \rightarrow \mathcal{M}(X, C, r, n)$ forgetting the data $(L, s)$. It is straightforward to check that the stabilizers of all closed points of $\mathcal{M}(X, C, r, n)$ are isomorphic to $\mathbb{C}^{\times}$.

\subsection{Relation to nested Hilbert schemes}

Suppose $C \subset \mathbb{P}^{2}$ is a reduced irreducible divisor with one singular point $p$, otherwise smooth. For any $l \in \mathbb{Z}_{\geq 0}$ let $H^{l}(C)$ denote the Hilbert scheme of length $l$ zero-dimensional subschemes of $C$. Let $H^{[l, r]}(C) \subset H^{l}(C) \times$ $H^{l+r}(C)$ denote the cycle consisting of pairs of ideal sheaves $(J, I)$ such that

$$
m_{p} J \subseteq I \subseteq J
$$

where $m_{p} \subset \mathcal{O}_{C, p}$ is the maximal ideal in the local ring at $p$.

The main observation is that the nested Hilbert schemes $H^{[l, r]}(C)$, equipped with an appropriate scheme structure, are isomorphic to relative Quot schemes over $H^{l}(C)$. Let $\mathcal{J}$ denote the universal ideal sheaf on $H^{l}(C) \times C$ and $\mathcal{J}_{p}$ its restriction to the closed subscheme $H^{l}(C) \times\{p\}$. Let $Q^{[l, r]}(C)$ the relative Quot-scheme parametrizing rank $r$ locally free quotients of $\mathcal{J}_{p}$ over $H^{l}(C)$. Standard results on Quot-schemes show that $Q^{[l, r]}(C)$ is a quasi-projective scheme over $H^{l}(C)$. Note that a closed point of $Q^{[l, r]}(C)$ over a closed point $[J] \in H^{l}(C)$ is a pair $(V, \xi)$ where $V$ is a $r$-dimensional vector space over $\mathbb{C}$ and $\xi: J \otimes \mathcal{O}_{p} \rightarrow V$ is a surjective map of complex vector spaces. In particular, the fiber of $Q^{[l, r]}(C)$ is empty if $J$ has less than $r$ generators at $p$. Let

$$
I=\operatorname{Ker}\left(J \rightarrow J \otimes \mathcal{O}_{C, p} \stackrel{\xi}{\longrightarrow} V\right) .
$$

Then it is straightforward to check that $(J, I)$ is a pair of ideal sheaves on $C$ satisfying conditions (3.14) at $p$. Note that the resulting scheme structure on $H^{[l, r]}(C)$ may be different from the reduced induced scheme structure.

The main result of this section is:

Proposition 3.6. For any $(r, n) \in \mathbb{Z}_{\geq 1} \times \mathbb{Z}, n \geq \chi\left(\mathcal{O}_{C}\right)$, there is an isomorphism

$$
q: Q(X, C, r, n) \stackrel{\sim}{\longrightarrow} Q^{[l, r]}(C)
$$

over $H^{l}(C)$, where $l=n-\chi\left(\mathcal{O}_{C}\right)$. 
The first step in the proof of Proposition 3.6 is the observation that the moduli stack $\mathcal{Q}(X, C, r, n)$ admits a dual formulation which makes the connection with the Hilbert scheme of $C$ manifest. Let $J \subset \mathcal{O}_{C}$ be the ideal sheaf of a zero-dimensional subscheme of $C$ and consider an exact sequence of $\mathcal{O}_{X}$-modules

$$
0 \rightarrow V \otimes \mathcal{O}_{C_{0}}(-1) \rightarrow F \rightarrow J \rightarrow 0
$$

with $V$ a finite-dimensional vector space. The extension (3.16) is called nondegenerate if for any nontrivial quotient $V \rightarrow V^{\prime}$, the corresponding extension class $e$ is not in the kernel of the natural map

$$
\operatorname{Ext}_{X}^{1}\left(J, V \otimes \mathcal{O}_{C_{0}}(-1)\right) \rightarrow \operatorname{Ext}_{X}^{1}\left(J, V^{\prime} \otimes \mathcal{O}_{C_{0}}(-1)\right)
$$

Now let $\pi: \mathcal{Q}^{*}(X, C, r, l) \rightarrow H^{l}(C)$ be a moduli stack over $H^{l}(C)$ defined as follows. For any scheme $\tau: T \rightarrow H^{l}(C)$ let $J_{T}$ be the flat family of ideal sheaves on $C$ obtained by pull-back. The objects of $\mathcal{Q}^{*}(X, C, r, l)$ over $\tau$ : $T \rightarrow H^{l}(C)$ are collections $\left(\mathcal{V}_{T}, F_{T}, f_{T}, g_{T}\right)$ where $V_{T}$ is a locally free $\mathcal{O}_{T^{-}}$ module, $F_{T}$ is a flat family of pure dimension one sheaves on $X$, and $g_{T}$ : $\pi_{T}^{*} \mathcal{V}_{T} \otimes_{X_{T}} \mathcal{O}_{C_{0 T}}(-1) \rightarrow F_{T}, h_{T}: F_{T} \rightarrow J_{T}$ are morphisms of $\mathcal{O}_{X_{T}}$-modules such that

$\left(a^{*}\right)$ For any closed point $t \in T$ there is an exact sequence of $\mathcal{O}_{X_{t}}$-modules

$$
0 \rightarrow V_{t} \otimes_{X_{t}} \mathcal{O}_{C_{0 t}}(-1) \stackrel{g_{t}}{\longrightarrow} F_{t} \stackrel{h_{t}}{\longrightarrow} J_{t} \rightarrow 0
$$

$\left(b^{*}\right)$ The extension (3.17) is nondegenerate.

Isomorphisms are defined naturally. Then the following holds.

Lemma 3.7. For any $(r, n) \in \mathbb{Z}_{\geq 0} \times \mathbb{Z}, n \geq \chi\left(\mathcal{O}_{C}\right)$, there is an isomorphism $j: \mathcal{Q}(X, C, r, n) \stackrel{\sim}{\longrightarrow} \mathcal{Q}^{*}(X, C, r, l), l=n-\chi\left(\mathcal{O}_{C}\right)$.

Proof. Given any collection $(V, L, F, s, f)$ satisfying conditions $(a)-(d)$, let $G=\operatorname{Ker}(f)$. Note that the section $s: \mathcal{O}_{X} \rightarrow F$ must factor through $s_{C}$ : $\mathcal{O}_{C} \rightarrow G$ since $H^{0}\left(\mathcal{O}_{C_{0}}(-1)\right)=0$ and $G$ is scheme theoretically supported on $C$. According to [46, Prop. B8], the moduli space of pairs $\left(G, s_{C}\right)$ is isomorphic to the Hilbert scheme $H^{l}(C)$. The isomorphism is obtained by taking the derived dual $G^{\vee}=R \operatorname{Hom}_{C}\left(G, \mathcal{O}_{C}\right)$, which is an ideal sheaf on $C$. 
The isomorphism $j$ will be first constructed on closed points. Note that taking derived duals on $X$ one obtains an exact sequence

$$
0 \rightarrow V^{\vee} \otimes \mathcal{O}_{C_{0}}(-1) \stackrel{f^{\vee}}{\longrightarrow} \mathcal{E} x t^{2}\left(F, \mathcal{O}_{X}\right) \rightarrow \mathcal{E} x t^{2}\left(G, \mathcal{O}_{X}\right) \rightarrow 0
$$

The duality theorem for the closed embedding $\iota: C \hookrightarrow X$ yields an isomorphism

$$
R \iota_{*} R \mathcal{H} \operatorname{som}_{C}\left(G, \omega_{C}\right)[-2] \simeq R \mathcal{H} \operatorname{com}_{X}\left(R \iota_{*} G, \mathcal{O}_{X}\right) .
$$

Note also that $\omega_{C} \simeq \mathcal{O}_{X}((k-3) H)$, where $k \in \mathbb{Z}_{>0}$ is the degree of $C \subset D$. Therefore there is an isomorphism of $\mathcal{O}_{X}$-modules

$$
\iota_{*} G^{\vee} \simeq \mathcal{E} x t_{X}^{2}\left(G, \mathcal{O}_{X}\right) \otimes_{X} \mathcal{O}_{X}((3-k) H)
$$

where $G^{\vee}$ denotes the derived dual on $C$. Moreover, it is straightforward to check that the extension:

$$
0 \rightarrow G \rightarrow F \stackrel{f}{\longrightarrow} V \otimes \mathcal{O}_{C_{0}}(-1) \rightarrow 0
$$

is nondegenerate if and only if the dual (3.18) is nondegenerate.

In conclusion, the functor $j$ has been constructed on closed points. The construction in families is analogous, using [46, Prop. B.8].

In order to conclude the proof of Proposition 3.6, recall that according to Lemma 2.6 there is an isomorphism

$$
\varphi_{1}: \operatorname{Ext}_{X}^{1}\left(J, V \otimes \mathcal{O}_{C_{0}}(-1)\right) \stackrel{\sim}{\longrightarrow} \operatorname{Hom}_{D}\left(J, V \otimes \mathcal{O}_{p}\right) .
$$

Moreover, Corollary 2.7 shows that for given a morphism $\psi: J \rightarrow V \otimes \mathcal{O}_{p}$, the extension

$$
0 \rightarrow V \otimes \mathcal{O}_{C_{0}}(-1) \rightarrow F_{\varphi_{1}^{-1}(\psi)} \rightarrow J \rightarrow 0
$$

is nondegenerate if and only if $\psi$ is surjective. Now note that there is an isomorphism

$$
\operatorname{Hom}_{C}\left(J, V \otimes \mathcal{O}_{p}\right) \simeq \operatorname{Hom}_{C}\left(J \otimes_{C} \mathcal{O}_{p}, V \otimes \mathcal{O}_{p}\right), \quad \psi \mapsto \bar{\psi}
$$

such that $\psi$ is surjective if and only if $\bar{\psi}$ is surjective. Then Proposition 3.6 follows from Lemma 3.7 by a straightforward comparison of flat families.

Remark 3.8. Note that Proposition 3.6 implies that the stack $\mathcal{Q}(X, C, r, n)$ is a $\mathbb{C}^{\times}$-gerbe over the $Q u$ ot schemes $Q^{[l, r]}(C)$. A similar result holds for the 
moduli stacks $\mathcal{M}(X, C, r, n)$ of decorated objects satisfying conditions $(a)$, (b), $(d)$ introduced in Remark 3.5. Let $\mathcal{M}^{[l, r]}(C)$ be the moduli stack of pairs $(J, \psi)$ where $J$ is any abstract sheaf which admits an isomorphism to a length $l$ ideal sheaf on $C$, and $\psi: J \rightarrow \mathcal{O}_{p}^{\oplus r}$ a surjective morphism. Two such pairs are isomorphic if there is an isomorphism of sheaves $\xi: J \rightarrow J^{\prime}$ such that $\psi^{\prime} \circ \xi=\psi$. By analogy with the moduli spaces $Q^{[l, r]}(C), M^{[l, r]}(C)$ are naturally identified with relative Quot schemes over the compactified Jacobian of $C$ of degree $l=n-\chi\left(\mathcal{O}_{C}\right)$. By analogy with Proposition 3.6 the stacks $\mathcal{M}(X, C, r, n)$ are $\mathbb{C}^{\times}$-gerbes over the moduli spaces $M^{[l, r]}(C)$. Moreover there is an obvious forgetful morphism $\pi: Q^{[l, r]}(C) \rightarrow M^{[l, r]}(C)$ determined by the natural morphism from the Hilbert scheme to the compactified Jacobian.

Let $\mathcal{N}(D, k, r, n)$ be the moduli stack of pairs $(J, \psi)$ where $J$ is a rank one torsion free sheaf on a degree $k$ reduced irreducible divisor on $D$ and $\psi: J \rightarrow \mathcal{O}_{p}^{\oplus r}$ a surjective morphism. Obviously there is a natural projection $\mathcal{N}(D, k, r, n) \rightarrow \mathcal{U}$ to an open subset of the linear system $\mathbb{P}\left(H^{0}\left(I_{p}(k H)\right)\right)$ where $I_{p}$ is the maximal ideal sheaf of $p . M^{[l, r]}(C)$ is the fiber of this projection over the point $[C] \in \mathcal{U}$. Since any $\mathcal{O}_{D}$-module $J$ as above is automatically slope and Gieseker stable on $D$, one can easily check that such a pair $(J, \psi)$ is $\delta$-stable in the sense of [18] for sufficiently small $\delta>0$. Then the results of [18] imply that $\mathcal{N}(D, k, r, n)$ is a quasi-projective moduli scheme.

Lemma 3.9. If $r<3 k, \mathcal{N}(D, k, r, n)$ is smooth.

Proof. According to [18], the deformation theory of a pair $(J, \psi)$ is determined by the extension groups $\operatorname{Ext}_{D}^{k}(J, \mathrm{C}(\psi)[-1]), k=1,2$, where $\mathrm{C}(\psi)$ is the cone of $\psi$. In order to prove smoothness it suffices to show that $\operatorname{Ext}_{D}^{2}(J, \mathrm{C}(\psi)[-1])=0$. Since $\psi$ is surjective, $\mathrm{C}(\psi)[-1]$ is quasi-isomorphic to the kernel $I=\operatorname{Ker}(\psi)$. Let $T=\mathcal{O}_{p}^{\oplus r}$. Then there is an exact sequence

$$
\cdots \longrightarrow \operatorname{Ext}_{D}^{1}(J, J) \longrightarrow \operatorname{Ext}_{D}^{1}(J, T) \longrightarrow \operatorname{Ext}_{D}^{2}(J, I) \longrightarrow \cdots
$$

Since $J$ is a stable $\mathcal{O}_{D}$-module and $D \simeq \mathbb{P}^{2}$ is Fano, $\operatorname{Ext}^{2}(J, J)=0$. Therefore it suffices to prove that the natural map

$$
\psi_{*}: \operatorname{Ext}_{D}^{1}(J, J) \longrightarrow \operatorname{Ext}_{D}^{1}(J, T)
$$

is surjective. Using Serre duality on $D, \operatorname{Ext}_{D}^{2}(J, I) \simeq \operatorname{Ext}^{0}\left(I, J \otimes_{D} \omega_{D}\right)^{\vee}$. Since $I, J$ are both slope stable on $D$ with $\operatorname{ch}_{1}(I)=\operatorname{ch}_{1}(J)=k H$, this group 
is trivial if $\chi(I)>\chi\left(J \otimes_{D} \omega_{D}\right)$. However,

$$
\chi(I)=\chi(J)-\chi(T)=\chi(J)-r, \quad \chi\left(J \otimes_{D} \omega_{D}\right)=\chi(J)-3 k .
$$

Therefore, the conclusion follows if $r<3 k$.

Remark 3.10. Note that

$$
\operatorname{Hom}_{C}\left(J, \mathcal{O}_{p}^{\oplus r}\right) \simeq \operatorname{Hom}\left(J \otimes_{C} \mathcal{O}_{p}, \mathbb{C}^{r}\right)
$$

Therefore the existence of a surjective morphism $\psi: J \rightarrow \mathcal{O}_{p}^{\oplus r}$ requires $r$ to be smaller than the minimal number of generators of $J$ at $p, m(J)=$ $\operatorname{dim}\left(J \otimes_{D} \mathcal{O}_{p}\right)$. However this number is bounded above ${ }^{1}$ by the degree $k$ of $C$, therefore the condition $r<3 k$ is always satisfied.

\subsection{Relation to small $b$ moduli spaces}

Let $\mathcal{P}_{0+}(X, C, r, n)$ denote the moduli stack of $\mu_{(\omega, b)}$-slope stable objects of $\mathcal{A}^{C}$, where $b$ satisfies the bound (3.1). By analogy with $[50,51], \mathcal{P}_{0+}(X, C, r, n)$ is an algebraic stack of finite type over $\mathbb{C}$, and all stabilizers of closed points are isomorphic to $\mathbb{C}^{\times}$. Recall that an object of $\mathcal{P}_{0+}(X, C, r, n)$ is a perfect complex $E_{T}$ on $X_{T}$ such that its restriction $L \iota_{t}^{*} E_{T}$ is a $\mu_{(\omega, b)}$-slope stable object of the category $\mathcal{A}^{C}$ associated to the fiber $X_{t}$ for any closed point $\iota_{t}:\{t\} \hookrightarrow T$. In this subsection $b>0$ will be a small stability parameter of type $(r, n)$ satisfying the bound $(3.1)$.

Any flat family $\left(V_{T}, L_{T}, F_{T}, s_{T}, f_{T}\right)$ over $T$, determines a complex

$$
E_{T}=\left(\pi_{T}^{*} L_{T} \stackrel{s_{T}}{\longrightarrow} F_{T}\right)
$$

on $X_{T}$. Since $F_{T}$ is flat over $T$, and $X_{T}$ is smooth projective over $T, E_{T}$ is perfect. Moreover, the derived restriction of $E_{T}$ to any closed fiber $X_{t}$ is simply obtained by restricting the terms of $E_{T}$ to $X_{t}$. It follows that the complex $L \iota_{t}^{*} E_{T}$ satisfies the conditions of Proposition 3.3. Therefore this construction defines a morphism of stacks

$$
f: \mathcal{Q}(X, C, r, n) \rightarrow \mathcal{P}_{0+}(X, C, r, n)
$$

\footnotetext{
${ }^{1}$ We thank Vivek Shende for pointing out this bound.
} 
Proposition 3.11. The morphism $f$ is geometrically bijective i.e., it yields an equivalence

$$
f(\mathbb{C}): \mathcal{Q}(X, C, r, n)(\mathbb{C}) \stackrel{\sim}{\longrightarrow} \mathcal{P}_{0+}(X, C, r, n)(\mathbb{C})
$$

of groupoids of $\mathbb{C}$-valued points.

Proof. Proposition 3.3 and Remark 3.4 imply that any object of $\mathcal{P}_{0+}$ $(X, C, r, n)(\mathbb{C})$ is quasi-isomorphic to an object in the image of $f(\mathbb{C})$. One has to prove that if two data $(V, L, F, s, f)$ and $\left(V^{\prime}, L^{\prime}, F^{\prime}, s^{\prime}, f^{\prime}\right)$ are mapped to quasi-isomorphic complexes $E, E^{\prime}$ then they must be isomorphic. This can be proven by analogy with [45, Prop. 1.21]. Given an object $E$ of $\mathcal{A}^{C}$ satisfying the conditions of Proposition 3.3 there is an exact triangle

$$
\mathcal{O}_{X} \stackrel{s}{\longrightarrow} F \rightarrow E
$$

in $D^{b}(X)$ where $F$ is a nondegenerate extension

$$
0 \rightarrow G \rightarrow F \rightarrow V \otimes \mathcal{O}_{C_{0}}(-1) \rightarrow 0
$$

This yields a long exact sequence

$$
\begin{aligned}
\cdots & \rightarrow \operatorname{Hom}\left(F, \mathcal{O}_{X}\right) \rightarrow \operatorname{Hom}\left(\mathcal{O}_{X}, \mathcal{O}_{X}\right) \rightarrow \operatorname{Hom}\left(E, \mathcal{O}_{X}[1]\right) \\
& \rightarrow \operatorname{Hom}\left(F, \mathcal{O}_{X}[1]\right) \rightarrow \cdots
\end{aligned}
$$

The first term is obviously trivial since $F$ is torsion and Serre duality implies that

$$
\operatorname{Hom}\left(F, \mathcal{O}_{X}[1]\right) \simeq H^{2}(F)^{\vee}=0,
$$

since $F$ is supported in dimension at most one. Therefore

$$
\operatorname{Hom}\left(E, \mathcal{O}_{X}[1]\right) \simeq \operatorname{Hom}\left(\mathcal{O}_{X}, \mathcal{O}_{X}\right) \simeq \mathbb{C}
$$

which implies that there is a unique morphism $E \rightarrow \mathcal{O}_{X}[1]$ up to multiplication by nonzero complex numbers. Then $F$ is quasi-isomorphic to the mapping cone of the morphism $E \rightarrow \mathcal{O}_{X}[1]$, and the section $s$ is recovered from the induced map $\mathcal{O}_{X} \rightarrow F$ as in [45, Prop. 1.21]. In order to finish the proof, note that given two extensions $F, F^{\prime}$ of the form (3.19), an isomorphism of sheaves $F \stackrel{\sim}{\longrightarrow} F^{\prime}$ induces isomorphisms $G \stackrel{\sim}{\longrightarrow} G^{\prime}$, respectively $V \stackrel{\sim}{\longrightarrow} V^{\prime}$ using the snake lemma. Therefore, the data $(V, L, F, s, f)$ can be recovered up to isomorphism from the complex $E$. 
In conclusion, note that Propositions 3.6 and 3.11 imply Theorem 1.3 as follows.

Proof of Theorem 1.3. Summarizing the results of Propositions 3.6 and 3.11, the moduli stack $\mathcal{P}_{0+}(X, C, r, n)$ of $C$-framed perverse coherent sheaves in the small $b>0$ chamber is geometrically bijective with the moduli stack of decorated sheaves $\mathcal{Q}(X, C, r, n)$. The latter is in turn an $\mathbb{C}^{\times}$-gerbe over the relative Quot scheme $Q^{[l, r]}(C), l=n-\chi\left(\mathcal{O}_{C}\right)$, introduced above Proposition 3.6. Then, using [24, Sect. 3.5, Eqn. (43)], and [24, Thm. 3.16], the topological Euler character invariants $P_{0+}^{\mathrm{top}}(X, C, r, n)$ are given by

$$
P_{0+}^{\text {top }}(X, C, r, n)=\chi\left(Q^{[l, r]}(C)\right)
$$

Next let $\pi: Q^{[l, r]}(C) \rightarrow H^{l}(C)$ be the canonical projection to the Hilbert scheme. Let $J$ denote the universal ideal sheaf on $H^{l}(C) \times C$. By construction, there is a universal quotient

$$
\left.\left(\pi \times 1_{C}\right)^{*} J\right|_{Q^{[l, r]}(C) \times\{p\}} \rightarrow \mathcal{V},
$$

where $\mathcal{V}$ is a rank $r$ locally free sheaf on $Q^{[l, r]} \times\{p\}$. Therefore there is a surjective morphism

$$
\left(\pi \times 1_{C}\right)^{*} J \rightarrow \iota_{p *} \mathcal{V}
$$

of sheaves on $Q^{[l, r]}(C) \times C$, where $\iota_{p}: Q^{[l, r]} \times\{p\} \hookrightarrow Q^{[l, r]}(C) \times C$ denotes the canonical closed embedding. Moreover, both sheaves in (3.20) are flat over $Q^{[l, r]}(C)$. Therefore the kernel $\mathcal{I}$ of the morphism (3.20) is also flat over $Q^{[l, r]}(C)$, and the long exact sequence

$$
0 \rightarrow \mathcal{I} \rightarrow\left(\pi \times 1_{C}\right)^{*} J \rightarrow \iota_{p *} \mathcal{V} \rightarrow 0
$$

restricts to an exact sequence on each curve $\{q\} \times C$, with $q$ a closed point of $Q^{[l, r]}(C)$. This implies that $\mathcal{I}$ is a flat family of length $l+r$ ideal sheaves on $C$ parameterized by $Q^{[l, r]}(C)$. Hence it determines a morphism $\tau: Q^{[l, r]}(C) \rightarrow$ $H^{l+r}(C)$. Moreover, by construction, the image of the morphism $\pi \times \tau$ : $Q^{[l, r]}(C) \rightarrow H^{l}(C) \times H^{l+r}(C)$, equipped with the reduced induced scheme structure is the nested Hilbert scheme $H^{[l, r]}(C)$. 
In conclusion, the generating function

$$
Z_{0+}^{\mathrm{top}}(X, C, u, T)=\sum_{n \in \mathbb{Z}} \sum_{r \geq 0} T^{r} u^{n} P_{0+}^{\mathrm{top}}(X, C, r, n)
$$

is equal to

$$
u^{\chi\left(\mathcal{O}_{C}\right)} \sum_{l \geq 0} \sum r \geq 0 T^{r} u^{l} \chi\left(H^{[l, r]}(C)\right) .
$$

Now note that the Hilbert scheme $H^{l}(C)$ admits a stratification

$$
\cdots \subset H_{\geq s}^{l}(C, p) \subset \cdots \subset H_{\geq 1}^{l}(C, p)=H_{p}^{l}(C),
$$

where $H_{\geq s}^{l}(C, p), s \geq 1$ denotes the closed subscheme parameterizing ideal sheaves $I \subset \mathcal{O}_{C, p}$ with at least $s$ generators at $p$. Let $\mathcal{S}_{s}^{l}(C, p)=H_{\geq s}^{l}(C, p) \backslash$ $H_{\geq s+1}^{l}(C, p)$ denote the locally closed strata. Then the natural projection morphism $H^{[l, r]}(C) \rightarrow H^{l}(C)$ is a smooth $G r(s, r)$-fibration over the locally closed stratum $\mathcal{S}_{s}^{l}(C, p)$, where $G r(s, r)$ is the Grassmannian of $r$-dimensional quotients of $\mathbb{C}^{m}$. In particular, the fibers are empty over strata with $s<r$. This implies that

$$
\begin{aligned}
Z_{0+}^{\mathrm{top}}(X, C, u, T) & =u^{\chi\left(\mathcal{O}_{C}\right)} \sum_{l \geq 0} \sum_{r \geq 0} T^{r} u^{l} \sum_{s \geq r} \chi(G r(s, r)) \chi\left(\mathcal{S}_{s}^{l}(C, p)\right) \\
& =u^{\chi\left(\mathcal{O}_{C}\right)} \sum_{l \geq 0} \sum_{r \geq 0} T^{r} u^{l} \sum_{s \geq r}\left(\begin{array}{l}
s \\
r
\end{array}\right) \chi\left(\mathcal{S}_{s}^{l}(C, p)\right) \\
& =u^{\chi\left(\mathcal{O}_{C}\right)} \sum_{l \geq 0} u^{l} \int_{H^{l}(C)}(1+T)^{m} d \chi
\end{aligned}
$$

where $m: H^{l}(C) \rightarrow \mathbb{Z}$ is the constructible function which takes value $s$ on the stratum $\mathcal{S}_{s}^{l}(C, p)$. This implies Equation (1.15) in Theorem 1.3 making the substitutions $T=-a^{2}, u=q^{2}$.

\section{Motivic invariants at small $b$}

Composing the morphism $f: \mathcal{Q}(X, C, r, n) \rightarrow \mathcal{P}_{0+}(X, C, r, n)$ with the natural morphism $p: \mathcal{P}_{0+}(X, C, r, n) \rightarrow O b(\mathcal{A})$ one obtains a stack function

$$
q: \mathcal{Q}(X, C, r, n) \rightarrow O b(\mathcal{A})
$$

which determines an element of the motivic Hall algebra $H(\mathcal{A})$. The construction of $H(\mathcal{A})$ is briefly reviewed in A.2, as background material for 
the proof of Theorem 1.1. The motivic Donaldson-Thomas theory of [26] assigns to any stack function an invariant with values in a certain ring of motives, as reviewed below. Note that the formalism of [24] does not apply to abelian category of perverse coherent sheaves since no rigorous construction of holomorphic Chern-Simons functionals for such objects has been carried out yet in the literature. Therefore this section will rely on the conjectural construction of [26] employing motivic vanishing cycles for formal functions.

The goal of this section is to compare the resulting motivic invariants with the motivic Hilbert scheme series defined in Equation (1.11), Section 1.4. The two generating functions will be shown to agree subject to a conjectural comparison formula between the motivic weights of moduli stacks of stable pairs and sheaves. This is a natural motivic counterpart of previous results for numerical invariants [46], which will be proven here only for sheaves of sufficiently high degree. The general case is an open conjecture.

The required elements in the construction of motivic DT invariants after [26] are the integral identity conjectured in [26, Conj. 4, Section 4.4] and the orientation data [26, Section 5.2]. The integral identity has been recently proven in [49], therefore [26, Thm. 8 , Sect 6.3] yields a motivic integration map as soon as the derived category is equipped with orientation data. This will be assumed without proof in this paper. Moreover, explicit computations of motivic weights for sheaves will be carried out in Sections 4.3, 4.4 by reduction to a triangulated subcategory of quiver representations. In that context it will be further assumed that the orientation data on the ambient category $D^{b}(X)$ agrees with orientation data on the derived category of quiver representations constructed in $[9,27]$.

\subsection{Review of motivic DTs invariants}

Recall that if $X$ is a compact complex Calabi-Yau 3-fold then the derived category of coherent sheaves $D^{b}(X)$ carries a structure of 3D Calabi-Yau category (3CY category for short), see [26] for details. In particular, we endow it with a cyclic $A_{\infty}$-structure, for example by fixing a Calabi-Yau metric on $X$. Then according to [26, Sect 3] there is a formal potential function $W_{E}$ on the vector space $\operatorname{Hom}^{1}(E, E)$ for any object $E$ of $D^{b}(X)$. Replacing the category by its minimal model we can treat $W_{E}$ as a formal function on $\operatorname{Ext}^{1}(E, E)$. Moreover, [26, Prop. 7, Section 3.3] shows that there is a direct sum decomposition of formal functions

$$
W_{E}=W_{E}^{\min } \oplus Q_{E} \oplus N_{E},
$$


where

- $W_{E}^{\min }$ is the potential of the minimal $A_{\infty}$-model.

- $Q_{E}$ is a quadratic function on the quotient

$$
\operatorname{Ext}^{1}(E, E) / \operatorname{Ker}\left(m_{1}: \operatorname{Ext}^{1}(E, E) \rightarrow \operatorname{Ext}^{2}(E, E)\right) \text {. }
$$

- $N_{E}$ is the zero function on the image of $m_{1}: \operatorname{Ext}^{0}(E, E) \rightarrow \operatorname{Ext}^{1}(E, E)$.

The argument sketched in [26] shows that $D^{b}(X)$ is ind-constructible locally regular category. This means that $W_{E}$ can be treated as a regular function along the stack of objects of $D^{b}(X)$ and formal one in the "transversal" direction $\operatorname{Ext}^{1}(E, E)$. According to [26, Sect 4.3] $W_{E}$ determines a motivic Milnor fiber $M F_{0}\left(W_{E}\right)$ at 0 , with values in an appropriate ring of motives. Note that the motivic Thom-Sebastiani theorem implies that

$$
\left(1-M F_{0}\left(W_{E}\right)\right)=\left(1-M F_{0}\left(W_{E}^{\min }\right)\right)\left(1-M F_{0}\left(Q_{E}\right)\right) .
$$

Furthermore, suppose that the category $D^{b}(X)$ is endowed with orientation data and a polarization such that the construction of motivic DT series in [26, Section 6] applies. In particular, to each object $E$ of $\mathcal{A}$ one assigns a motivic weight

$$
w_{E}=\mathbb{L}^{(E, E)_{\leq 1} / 2}\left(1-M F_{0}\left(W_{E}\right)\right) \mathbb{L}^{-\operatorname{rk}\left(Q_{E}\right) / 2} .
$$

Following the conventions of [26], given any two objects $E_{1}, E_{2}$, set

$$
\left(E_{1}, E_{2}\right)_{j}=\operatorname{dim}\left(\operatorname{Ext}^{j}\left(E_{1}, E_{2}\right)\right), \quad\left(E_{1}, E_{2}\right)_{\leq j}=\sum_{i \leq j}(-1)^{i} \operatorname{dim}\left(\operatorname{Ext}^{i}\left(E_{1}, E_{2}\right)\right)
$$

for any $j \in \mathbb{Z}$.

Since $O b(\mathcal{A}) \subset O b\left(D^{b}(X)\right)$ we can treat constructible families over $O b(\mathcal{A})$ as constructible families over $O b\left(D^{b}(X)\right)$. This gives a homomorphism at the level of stack functions and motivic Hall algebras (since $\mathcal{A}$ is a heart of $t$-structure there are no negative $\mathrm{Ext}^{i}$ between its objects). As a result, we can apply the formalism of [26] to the category of perverse coherent sheaves. The motivic invariant for a stack function $[\mathcal{X} \rightarrow O b(\mathcal{A})]$ is defined by integration of motivic weights, which is defined in [26, Section 4.4]. The result is encoded in the morphism $\Phi$ constructed in [26, Thm. 8, Section 6.3] from the motivic Hall algebra $H(\mathcal{A})$ to the quantum torus. Let $\Gamma$ denotes the intersection of the image of the Chern character ch $: K_{0}\left(D^{b}(X)\right) \rightarrow H^{e v}(X, \mathbb{Q})$ with $H^{e v}(X, \mathbf{Z})$ (instead of $\Gamma$ one can take 
the quotient of $K_{0}(\mathcal{A})$ by the subgroup generated by the numerical equivalence). In particular, the lattice $\Gamma$ is equipped with a natural nondegenerate antisymmetric pairing $\langle$,$\rangle . The quantum torus is the associative algebra \mathcal{R}$ over an appropriate motivic ring described in [26] spanned by the symbols $\hat{e}_{\gamma}, \gamma \in \Gamma$ over the ring of motivic weights, where

$$
\hat{e}_{\gamma_{1}} \hat{e}_{\gamma_{2}}=\mathbb{L}^{\left\langle\gamma_{1}, \gamma_{2}\right\rangle / 2} \hat{e}_{\gamma_{1}+\gamma_{2}}
$$

Here $\mathbb{L}$ denotes the motive of the affine line.

Then the integration map $\Phi: H(\mathcal{A}) \rightarrow \mathcal{R}$ assigns to a stack function $[\mathcal{Y} \stackrel{\pi}{\longrightarrow} O b(\mathcal{A})]$, which factors through the substack $O b_{\gamma}(\mathcal{A})$, the element

$$
\int_{\mathcal{Y}} w_{\pi(y)} \hat{e}_{\gamma}
$$

\subsection{Motivic weights at small $b$}

The next goal is to evaluate the integration map $\Phi$ on the stack function

$$
q=p \circ f: \mathcal{Q}(X, C, r, n) \hookrightarrow O b(\mathcal{A})
$$

determined by Proposition 3.11. As observed in Remark 3.5, there is a natural forgetful morphism $\pi: \mathcal{Q}(X, C, r, n) \rightarrow \mathcal{M}(X, C, r, n)$ to the stack of nondegenerate extensions $(V, F, f)$. The fiber of $\pi$ over a closed point $(V, F, f)$ is isomorphic to the projective space $\mathbb{P} H^{0}(F)$. Note that there is also a natural obvious morphism $m: \mathcal{M}(X, C, r, n) \rightarrow O b(\mathcal{A})$ sending the sheaf $F$ to itself. Then the integration of motivic weights may be carried out in two stages, first along the fibers of $\pi$, and then then on $\mathcal{M}(X, C, r, n)$. The first step will be considered below, while the second one will be postponed for Section 4.3.

Note that there is a one-to-one correspondence between nonzero sections in $H^{0}(F)$ and nontrivial extensions

$$
0 \rightarrow F \rightarrow E \rightarrow \mathcal{O}_{X}[1] \rightarrow 0
$$

in $\mathcal{A}$. Set $E_{1}=F, E_{2}=\mathcal{O}_{X}[1]$ and $E_{0}=E_{1} \oplus E_{2}$. According to [26, Thm. 8 , Section 6.3], there is a relation

$$
\Phi\left(E_{1} \cdot E_{2}\right)=\Phi\left(E_{1}\right) \Phi\left(E_{2}\right)
$$


in the motivic quantum torus. As shown in Step 1 in the proof of loc. cit., this identity is equivalent to

$$
\int_{\alpha \in \operatorname{Ext}^{1}\left(E_{2}, E_{1}\right)} w_{E_{\alpha}}=\mathbb{L}^{\left[\left(E_{0}, E_{0}\right)_{\leq 1}-\left(E_{1}, E_{1}\right)_{\leq 1}-\left(E_{2}, E_{2}\right)_{\leq 1}\right] / 2} \mathbb{L}^{\left(E_{2}, E_{1}\right)_{1}} w_{E_{1}} w_{E_{2}},
$$

where $E_{\alpha}=$ Cone $(\alpha)$ for any extension class $\alpha \in \operatorname{Hom}\left(E_{2}[-1], E_{1}\right)=$ $\operatorname{Hom}\left(\mathcal{O}_{X}, F\right)$. In particular, for $\alpha=0$ one obtains the trivial extension, $E_{1} \oplus E_{2}$, which has been denoted by $E_{0}$ in the above equation.

Note that $\left(E_{2}, E_{2}\right)_{\leq 1}=1$ and $w_{E_{2}}=\mathbb{L}^{1 / 2}$ since $\mathcal{O}_{X}[1]$ is a spherical object. Since the fibers of $\pi$ parameterize nonzero extensions, integration along the fiber yields

$$
\int_{\alpha \in \operatorname{Ext}^{1}\left(E_{2}, E_{1}\right)} w_{E_{\alpha}}-w_{E_{0}}=\mathbb{L}^{\left[\left(E_{0}, E_{0}\right)_{\leq 1}-\left(E_{1}, E_{1}\right)_{\leq 1}\right] / 2} \mathbb{L}^{\left(E_{2}, E_{1}\right)_{1}} w_{E_{1}}-w_{E_{0}} .
$$

Now recall that the sheaves $F$ are nondegenerate extensions of the form

$$
0 \rightarrow G \rightarrow F \rightarrow V \otimes \mathcal{O}_{C_{0}}(-1) \rightarrow 0
$$

where $G$ is a rank one torsion free sheaf on a reduced irreducible divisor $C \subset D$. The motivic weight $w_{E_{0}}$ will be computed below in those cases where $H^{1}(G)=0$. Let $p: \operatorname{Ext}_{X}^{1}\left(E_{0}, E_{0}\right) \rightarrow \operatorname{Ext}_{X}^{1}(F, F)$ be the canonical projection. Let $W_{E_{0}}, W_{F}$ be the formal potential functions on $\operatorname{Ext}^{1}\left(E_{0}, E_{0}\right), \operatorname{Ext}^{1}(F, F)$, respectively.

Lemma 4.1. If $H^{1}(G)=0, W_{E_{0}}=p^{*} W_{F}$.

Proof. The proof will be based on [26, Thm. 9, Section 8]. Set $E_{1}=F$, $E_{2}=\mathcal{O}_{X}[1]$. One then has to check that the following conditions are satisfied

(a) $\operatorname{Ext}^{0}\left(E_{i}, E_{i}\right)=\mathbb{C} 1_{E}, i=1,2$,

(b) $\operatorname{Ext}^{0}\left(E_{i}, E_{j}\right)=0, i, j=1,2, i \neq j$,

(c) $\operatorname{Ext}^{<0}\left(E_{i}, E_{j}\right)=0, i, j=1,2$.

Condition $(a)$ is satisfied because the automorphism group of a nondegenerate extension $F$ is $\mathbb{C}^{\times} 1_{F}$. Condition $(b)$ is satisfied since

$$
\operatorname{Ext}^{0}\left(F, \mathcal{O}_{X}[1]\right) \simeq \operatorname{Ext}^{2}\left(\mathcal{O}_{X}, F\right)^{\vee} \simeq H^{2}(F)^{\vee}=0
$$


Condition $(c)$ is also satisfied since

$$
\operatorname{Ext}^{-1}\left(F, \mathcal{O}_{X}[1]\right)=\operatorname{Ext}^{0}\left(F, \mathcal{O}_{X}\right)=0 .
$$

Moreover, note that

$$
\operatorname{Ext}^{k}\left(\mathcal{O}_{X}, \mathcal{O}_{C_{0}}(-1)\right) \simeq H^{k}\left(\mathcal{O}_{C_{0}}(-1)\right)=0
$$

for all $k \in \mathbb{Z}$, hence

$$
\operatorname{Ext}^{k}\left(\mathcal{O}_{X}, F\right) \simeq \operatorname{Ext}^{k}\left(\mathcal{O}_{X}, G\right),
$$

for all $k \in \mathbb{Z}$. Using Serre duality,

$$
\operatorname{Ext}^{1}\left(G, \mathcal{O}_{X}[1]\right) \simeq \operatorname{Ext}^{1}\left(\mathcal{O}_{X}, G\right)^{\vee} \simeq H^{1}(G)^{\vee}=0 .
$$

Therefore, the $\operatorname{Ext}^{1}$ quiver of the collection of objects $\left\{E_{1}, E_{2}\right\}$ is of the form

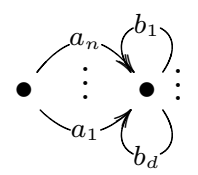

where $n=\operatorname{dim}\left(\operatorname{Ext}^{1}\left(E_{2}, E_{1}\right)\right) \simeq H^{0}(G)$, and $d=\operatorname{dim}\left(\operatorname{Ext}^{1}\left(E_{1}, E_{1}\right)\right)$. Note that there are no left directed arrows, hence all polynomial invariants of any quiver representation are determined by paths of the form $b_{i_{1}} b_{i_{2}} \cdots b_{i_{j}}$. Then [26, Thm. 9, Section 8] implies that $W_{E_{0}}=p^{*} W_{F}$.

Since $E_{2}=\mathcal{O}_{X}[1]$, it follows that Lemma 4.1 yields

$$
w_{E_{0}}=\mathbb{L}^{\left[\left(E_{0}, E_{0}\right)_{\leq 1}-\left(E_{1}, E_{1}\right)_{\leq 1}\right] / 2} w_{F}
$$

when the conditions of Lemma 4.1 are satisfied. Note that

$$
\begin{aligned}
\left(E_{0}, E_{0}\right)_{\leq 1}-\left(E_{1}, E_{1}\right)_{\leq 1}= & \left(\mathcal{O}_{X}[1], \mathcal{O}_{X}[1]\right)_{\leq 1}+\left(\mathcal{O}_{X}[1], F\right)_{\leq 1}+\left(F, \mathcal{O}_{X}[1]\right)_{\leq 1} \\
= & \left(\mathcal{O}_{X}[1], \mathcal{O}_{X}[1]\right)_{\leq 1}-\left(\mathcal{O}_{X}, F\right)_{\leq 0}-\left(F, \mathcal{O}_{X}\right)_{\leq 2} \\
= & \operatorname{dim} H^{0}\left(\mathcal{O}_{X}\right)-\operatorname{dim} H^{0}(F) \\
& -\sum_{i=0}^{2}(-1)^{i} \operatorname{dimExt}^{i}\left(F, \mathcal{O}_{X}\right) \\
= & 1-\operatorname{dim} H^{0}(F)+\operatorname{dimExt}^{1}\left(F, \mathcal{O}_{X}\right) \\
& -\operatorname{dimExt}^{2}\left(F, \mathcal{O}_{X}\right) \\
= & 1-\operatorname{dim}^{0}(F)+\operatorname{dim} H^{2}(F)-\operatorname{dim} H^{1}(F)
\end{aligned}
$$


where Serre duality has been used at the last step. Under the conditions of Lemma 4.1,

$$
\operatorname{dim} H^{0}(F)=n, \quad \operatorname{dim} H^{1}(F)=0
$$

Therefore

$$
\left(E_{0}, E_{0}\right)_{\leq 1}-\left(E_{1}, E_{1}\right)_{\leq 1}=1-n .
$$

If this is the case, Equation (4.2) yields

$$
\int_{\alpha \in \operatorname{Ext}^{1}\left(E_{2}, E_{1}\right)} w_{E_{\alpha}}-w_{E_{0}}=\mathbb{L}^{(1-n) / 2}\left(\mathbb{L}^{n}-1\right) w_{F} .
$$

In particular, this holds for all sheaves $F$ for sufficiently large $n$. By analogy with [46, Thm. 4] it is natural to conjecture that the following holds for general $n \in \mathbb{Z}$

$$
\int_{\alpha \in \operatorname{Ext}^{1}\left(E_{2}, E_{1}\right)} w_{E_{\alpha}}-w_{E_{0}}=\mathbb{L}^{(1-n) / 2}\left(\mathbb{L}^{h^{0}(F)}-1\right) w_{F} .
$$

Using local toric models, the motivic weights $w_{F}$ will be represented below as of motivic Milnor fibers of polynomial Chern-Simons functions.

\subsection{Local toric models}

A straightforward local computation shows that the formal neighborhood of the union $D \cup C_{0}$ equipped with the reduced scheme structure is isomorphic to the formal neighborhood of an identical configuration in a toric CalabiYau threefold. This is in fact easier to see starting with with the small crepant resolution $X^{-} \rightarrow X_{0}$, related to $X \rightarrow X_{0}$ by a flop of the exceptional curve. For the elliptic fibration example given in Section $1.4, X^{-}$is a smooth elliptic fibration with canonical section over the Hirzebruch surface $\mathbb{F}_{1}$. The exceptional curve $C_{0}^{-}$is contained in the section $D^{-}$, which is identified with $\mathbb{F}_{1}$. Then the formal neighborhood of $D^{-}$in $X^{-}$is isomorphic to the formal neighborhood of the zero section in the total space $Z^{-}$of the canonical bundle $K_{\mathbb{F}_{1}}$. Moreover $D^{-}$is identified with the zero section and $C_{0}^{-}$is identified with the unique $(-1)$-curve on $Z^{-}$. Then one can construct a second smooth toric Calabi-Yau threefold $Z^{+}$related to $Z^{-}$by a toric flop along the curve $C_{0}^{-}$as shown in detail below. This threefold contains a compact divisor $D^{+} \simeq \mathbb{P}^{2}$ and an exceptional $(-1,-1)$ curve $C_{0}^{+}$intersecting $D^{+}$transversely at a point $p$. 

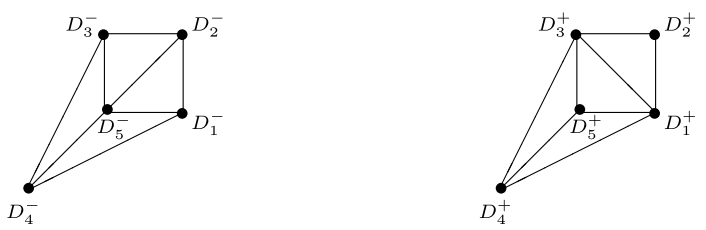

Figure 1: Local toric models related by a flop. The polytope on the left is the $z=1$ section of the toric fan of the local $\mathbb{F}_{1}$ model. The polytope on the right is a similar section of the toric fan of the local $\mathbb{P}^{2} \cup \mathbb{P}^{1}$ model. The two models are related by a toric flop corresponding to the obvious change of triangulation.

The toric presentation of both $Z^{ \pm}$is of the form

$\begin{array}{ccccc}x_{1} & x_{2} & x_{3} & x_{4} & x_{5} \\ 1 & 0 & 1 & 1 & -3 \\ 0 & 1 & 0 & 1 & -2 .\end{array}$

The disallowed locus is $\left\{x_{1}=x_{3}=0\right\} \cup\left\{x_{2}=x_{4}=0\right\}$ for $Z^{-}$and $\left\{x_{1}=\right.$ $\left.x_{3}=x_{4}\right\} \cup\left\{x_{2}=x_{4}=0\right\}$ for $Z^{-}$. The toric fans $\nabla^{ \pm}$of $Z^{ \pm}$are generated by the vectors

$$
\begin{aligned}
& v_{1}=(1,0,1), \quad v_{2}=(1,1,1), \quad v_{3}=(0,1,1), \\
& v_{4}=(-1,-1,1), \quad v_{5}=(0,0,1)
\end{aligned}
$$

in $\mathbb{R}^{3}$. In each case the fan is a cone over a $2 \mathrm{D}$ polytope embedded in the plane $z=1 \mathrm{in} \mathbb{R}^{3}$. The toric flop relating $Z^{-}$and $Z^{+}$corresponds to a change of triangulation of the $2 \mathrm{D}$ polytopes, as shown in figure 1 .

The canonical toric divisors $x_{i}=0$ are denoted by $D_{i}^{ \pm}, i=1, \ldots, 5$. They are in one-to-one correspondence with the rays of the toric fans as shown in figure 1 . Note that $D^{ \pm}=D_{5}^{ \pm}$are the only compact divisors on $Z^{ \pm}$.

The derived categories of $Z^{ \pm}$are equivalent and are generated by line bundles. A collection of line bundles generating $D^{b}\left(Z^{-}\right)$is obtained by pulling back an exceptional collection on the Hirzebruch surface $\mathbb{F}^{1}$ of the form

$$
\mathcal{O}_{\mathbb{F}_{1}}, \quad \mathcal{O}_{\mathbb{F}_{1}}\left(C_{0}^{-}\right), \quad \mathcal{O}_{\mathbb{F}_{1}}(H), \quad \mathcal{O}_{\mathbb{F}_{1}}(2 H)
$$

Here $C_{0}^{-}$denotes the exceptional curve on $\mathbb{F}_{1}$ and $H$ the hyperplane class. Note that the resulting line bundles on $Z^{-}$are isomorphic to the toric line 
bundles

$$
\mathcal{O}_{Z^{-}}, \quad \mathcal{O}_{Z^{-}}\left(D_{2}^{-}\right), \quad \mathcal{O}_{Z^{-}}\left(D_{4}^{-}\right), \quad \mathcal{O}_{Z^{-}}\left(2 D_{4}^{-}\right)
$$

The direct sum $\mathcal{T}^{-}$of all above line bundles is a tilting object, and the derived category of $Z^{-}$is equivalent to the derived category of modules over the algebra $R \operatorname{End}_{Z^{-}}\left(\mathcal{T}^{-}\right)^{o p}$. The equivalence is given by the derived functor $R \operatorname{Hom}_{Z^{-}}\left(\mathcal{T}^{-}, \bullet\right)$. As a result the derived category of $Z^{-}$is equivalent to the $3 C Y$ category, which is a Calabi-Yau category associated with the abelian category $(Q, W)-\bmod$ of finite-dimensional representations of the following quiver $Q$

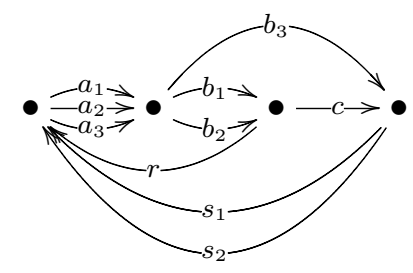

with potential

$$
W=r\left(b_{1} a_{2}-b_{2} a_{1}\right)+s_{1}\left(c b_{1} a_{3}-b_{3} a_{1}\right)+s_{2}\left(c b_{2} a_{3}-b_{3} a_{2}\right)
$$

Recall that this category can be described as the category of finitedimensional representations of the Jacobi algebra $\mathbb{C} Q /(\partial W)$, the quotient of the path algebra of $Q$ by the ideal generated by cyclic derivatives of $W$.

For future reference note that the line bundles

$$
\mathcal{O}_{D^{-}}, \quad \mathcal{O}_{D^{-}}\left(D_{2}^{-}\right), \quad \mathcal{O}_{D^{-}}\left(D_{4}^{-}\right), \quad \mathcal{O}_{D^{-}}\left(2 D_{4}^{-}\right)
$$

form an exceptional collection $\mathcal{T}_{D^{-}}$on the Hirzebruch surface $D^{-} \simeq \mathbb{F}_{1}$. The functor $R \operatorname{Hom}\left(\mathcal{T}_{D^{-}}, \bullet\right)$ yields an equivalence of the derived category $D^{b}\left(D^{-}\right)$ to the derived category of the abelian category $\left(Q_{0}, S\right)-\bmod$ of the finitedimensional representations of following quiver $Q_{0}$

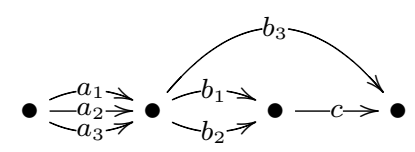

with relations

$S: \quad b_{1} a_{2}-b_{2} a_{1}, \quad c b_{1} a_{3}-b_{3} a_{1}, \quad c b_{2} a_{3}-b_{3} a_{2}$ 
The abelian category $\left(Q_{0}, S\right)-\bmod$ has homological dimension 2 , and there is an obvious injective fully faithful exact functor of abelian categories

$$
\iota:\left(Q_{0}, S\right)-\bmod \longrightarrow(Q, W)-\bmod
$$

For simplicity, extension groups in the two categories will be denoted by $\operatorname{Ext}_{\left(Q_{0}, S\right)}^{\bullet}, \operatorname{Ext}_{(Q, W)}^{\bullet}$ respectively. It will be useful to note that the following relations hold:

$$
\begin{aligned}
& \operatorname{Ext}_{(Q, W)}^{0}\left(\iota \rho_{1}, \iota \rho_{2}\right) \simeq \operatorname{Ext}_{\left(Q_{0}, S\right)}^{0}\left(\rho_{1}, \rho_{2}\right), \\
& \operatorname{Ext}_{(Q, W)}^{k}\left(\iota \rho_{1}, \iota \rho_{2}\right) \simeq \operatorname{Ext}_{\left(Q_{0}, S\right)}^{k}\left(\rho_{1}, \rho_{2}\right) \oplus \operatorname{Ext}_{\left(Q_{0}, S\right)}^{3-k}\left(\rho_{2}, \rho_{1}\right)^{\vee}, \quad k=1,2 .
\end{aligned}
$$

Using the results of [4], the direct sum $\mathcal{T}^{+}$of the following collection of line bundles

$$
\mathcal{L}_{1}=\mathcal{O}_{Z^{+}}\left(2 D_{4}^{+}\right), \quad \mathcal{L}_{2}=\mathcal{O}_{Z^{+}}\left(D_{4}^{+}\right), \quad \mathcal{L}_{3}=\mathcal{O}_{Z^{+}}\left(D_{2}^{+}\right), \quad \mathcal{L}_{4}=\mathcal{O}_{Z^{+}}
$$

is a tilting object in the derived category of $Z^{+}$. Therefore, it yields a similar equivalence of $D^{b}\left(Z^{+}\right)$to the derived category of the same quiver with potential.

The next step is to compute the image of dimension one sheaves on $Z^{+}$via the tilting functor. First note the following result which follows from [17, Lemm 9.1].

Lemma 4.2. Let $G$ be a rank one torsion-free sheaf on a degree $k \in \mathbb{Z}_{>0}$ reduced irreducible divisor on $D^{+} \simeq \mathbb{P}^{2}$ with $H^{0}(G)=0$. Then the complex $R \operatorname{Hom}\left(\mathcal{T}^{+}, G\right)[1]$ is quasi-isomorphic to a quiver representation $\rho_{G}$ of dimension vector

$$
v_{G}=(2 k-\chi(G), k-\chi(G),-\chi(G),-\chi(G))
$$

which belongs to the subcategory of $\left(Q_{0}, S\right)$-modules. Moreover

$$
\operatorname{Ext}_{\left(Q_{0}, S\right)}^{2}(G, G)=0
$$

and $\rho_{G}(c)$ is an isomorphism if $\chi(G) \neq 0$.

Proof. Note that the open subset $U=\left\{x_{2} \neq 0\right\} \subset Z^{+}$is isomorphic to the total space of the normal bundle $N_{D^{+} / Z^{+}} \simeq \omega_{\mathbb{P}^{2}}$. This follows observing that 
$U$ is isomorphic to a toric variety determined by the toric data

$$
\begin{array}{cccc}
x_{1}^{\prime} & x_{3}^{\prime} & x_{4}^{\prime} & x_{5}^{\prime} \\
1 & 1 & 1 & -3
\end{array}
$$

where

$$
x_{1}^{\prime}=x_{1}, \quad x_{3}^{\prime}=x_{3}, \quad x_{4}^{\prime}=x_{2}^{-1} x_{4} \quad x_{5}^{\prime}=x_{2}^{-2} x_{5}
$$

and the disallowed locus is $\left\{x_{1}^{\prime}=x_{3}^{\prime}=x_{4}^{\prime}=0\right\}$.

Denote the open immersion $U \hookrightarrow Z^{+}$by $j$ and the close immersions of $D^{+}$into $Z^{+}$and $U$ by $i$ and $i^{\prime}$ respectively. Clearly, $i=j \circ i^{\prime}$. Denote the tilting bundle on $Z^{+}$by $\mathcal{T}^{+}$. Given a sheaf on $Z^{+}$of the form $i_{*} G$, there is an isomorphism

$$
\operatorname{RHom}_{Z^{+}}\left(T, i_{*} G\right) \simeq \operatorname{RHom}_{U}\left(j^{*} T, i_{*}^{\prime} G\right)
$$

By adjunction, this is further isomorphic to $\operatorname{RHom}_{\mathbb{P}^{2}}\left(\mathcal{O}^{\oplus 2} \oplus \mathcal{O}(1) \oplus\right.$ $\mathcal{O}(2), G)$. By the derived Morita equivalence, this induces an equivalence between $D^{b}\left(\mathbb{P}^{2}\right)$ and the derived category of the abelian category $\mathcal{A}$ consisting of representations $\rho$ of the directed quiver $Q_{0}$ with dimension vectors $\left(v_{1}, v_{2}, v_{3}, v_{3}\right)$ and $\rho(c)$ an invertible linear map.

Since $\mathcal{A}$ is a fully faithful subcategory of $\left(Q_{0}, S\right)-\bmod$, we have

$$
\operatorname{Ext}_{\left(Q_{0}, S\right)}^{2}\left(\rho_{G}, \rho_{G}\right)=\operatorname{Ext}_{\mathcal{A}}^{2}\left(\rho_{G}, \rho_{G}\right)=\operatorname{Ext}_{\mathbb{P}^{2}}^{2}(G, G)=0
$$

when $G$ is stable.

The next goal is to compute the image of nondegenerate extensions

$$
0 \rightarrow G \rightarrow F \rightarrow V \otimes \mathcal{O}_{C_{0}^{+}}(-1) \rightarrow 0
$$

via the tilting functor. In order to obtain a single quiver representation as a opposed to a complex thereof, $F$ must be twisted by a suitable line bundle $L$ prior to tilting. There are several possible results depending on the choice of $L$. The one recorded below turns out to be most effective for the computation of motivic weights.

As shown in the proof of Lemma 3.7, taking derived duals on $X$ sends a nondegenerate extension as above to an extension of the form

$$
0 \rightarrow V^{\vee} \otimes \mathcal{O}_{C_{0}^{+}}(-1) \rightarrow \mathcal{E} x t_{Z^{+}}^{2}\left(F, \mathcal{O}_{Z^{+}}\right) \rightarrow J \otimes_{C} \omega_{C} \rightarrow 0
$$

where $J=R \operatorname{Hom}_{C}\left(G, \mathcal{O}_{C}\right)$ is an ideal sheaf on $C$. The dualizing sheaf of $C$ is $\left.\omega_{C} \simeq \mathcal{O}_{Z^{+}}\left((k-3) D_{4}^{+}\right)\right|_{C}$. Let $W=V^{\vee}$. The dual extension is also subject 
to a nondegeneracy condition. Namely the corresponding extension class $e \in \operatorname{Ext}^{1}\left(J \otimes_{C} \omega_{C}, W \otimes \mathcal{O}_{C_{0}^{+}}(-1)\right)$ is not in the kernel of the map

$$
\operatorname{Ext}^{1}\left(J \otimes_{C} \omega_{C}, W \otimes \mathcal{O}_{C_{0}^{+}}(-1)\right) \longrightarrow \operatorname{Ext}^{1}\left(J \otimes_{C} \omega_{C}, W^{\prime} \otimes \mathcal{O}_{C_{0}^{+}}(-1)\right)
$$

for any nontrivial quotient $W \rightarrow W^{\prime}$. The tilting functor will be applied to the twist $\left.F^{\prime}=\mathcal{E} x t_{Z^{+}}^{2}\left(F, \mathcal{O}_{Z^{+}}\right) \otimes_{Z^{+}}\left((2-k) D_{4}^{+}\right)\right)$which fits in an extension

$$
0 \rightarrow W \otimes \mathcal{O}_{C_{0}^{+}}(-1) \rightarrow F^{\prime} \rightarrow J\left(-D_{4}^{+}\right) \rightarrow 0
$$

Then the following holds:

Lemma 4.3. Consider a nondegenerate extension

$$
0 \rightarrow W \otimes \mathcal{O}_{C_{0}^{+}}(-1) \rightarrow F^{\prime} \rightarrow J^{\prime} \rightarrow 0
$$

where $J^{\prime}=J\left(-D_{4}^{+}\right)$for an ideal sheaf $J$ on a degree $k \in \mathbb{Z}_{>0}$ reduced irreducible divisor $C^{+}$on $D^{+} \simeq \mathbb{P}^{2}$. Then $R \operatorname{Hom}_{Z^{+}}\left(\mathcal{T}^{+}, F^{\prime}\right)[1]$ is quasiisomorphic to a quiver representation $\rho_{F}$ which fits in an extension

$$
0 \rightarrow W \otimes \rho_{3} \rightarrow \rho_{F} \rightarrow \rho_{J^{\prime}} \rightarrow 0
$$

In addition, $\rho_{F}$ belongs to the subcategory of $\left(Q_{0}, S\right)$-modules, and

$$
\operatorname{Ext}_{\left(Q_{0}, S\right)}^{2}\left(\rho_{F}, \rho_{F}\right)=0 .
$$

Proof. Observe that $\mathcal{O}_{C_{0}^{+}}(-1)$ is mapped to the simple module $\rho_{3}[-1]$ corresponding to the third vertex of the quiver $Q$. According to Lemma 4.2 the twisted derived dual $J^{\prime}$ of $G$ will be mapped to a representation $\rho_{J^{\prime}}[-1]$ of $Q_{0}$ since $H^{0}\left(J^{\prime}\right)=0$. Moreover, the linear map $\rho_{J^{\prime}}(c)$ is invertible. Then we claim

$$
\operatorname{Ext}_{\left(Q_{0}, S\right)}^{k}\left(\rho_{3}, \rho_{J^{\prime}}\right)=0
$$

for all $k \in \mathbb{Z}$. Suppose $\rho_{J^{\prime}}$ has dimension vector $d_{1}, \ldots, d_{4}$, recall that $\rho_{J^{\prime}}$ corresponds to a Maurer-Cartan element $x$ of the $L_{\infty}$ algebra $\operatorname{Ext}_{\left(Q_{0}, S\right)}^{*}\left(\oplus \rho_{i} \otimes\right.$ $\left.V_{i}, \oplus \rho_{i} \otimes V_{i}\right)$, where the dimension of $V_{i}$ equals $d_{i}$. The extension space $\operatorname{Ext}_{\left(Q_{0}, S\right)}^{*}\left(\rho_{3}, \oplus \rho_{i} \otimes V_{i}\right)$ is an $L_{\infty}$ module over $\operatorname{Ext}_{\left(Q_{0}, S\right)}^{*}\left(\oplus \rho_{i} \otimes V_{i}, \oplus \rho_{i} \otimes V_{i}\right)$. The Maurer-Cartan element $x$ defines a differential $\delta^{x}$ on $\operatorname{Ext}_{\left(Q_{0}, S\right)}^{*}\left(\rho_{3}, \oplus \rho_{i} \otimes\right.$ $V_{i}$ ) such that the cohomology groups compute $\operatorname{Ext}^{*}\left(\rho_{3}, \rho_{J^{\prime}}\right)$. The complex 
$\operatorname{Ext}_{\left(Q_{0}, S\right)}^{*}\left(\rho_{3}, \oplus \rho_{i} \otimes V_{i}\right)$ has the form

$$
0 \longrightarrow \operatorname{Hom}\left(\mathbb{C}, V_{3}\right) \stackrel{\delta^{x}}{\longrightarrow} \operatorname{Hom}\left(\mathbb{C}, V_{4}\right) \longrightarrow 0
$$

Since the linear map $\rho_{J^{\prime}}(c)$ is invertible, this complex is acyclic. For future reference, note that a similar argument proves that

$$
\operatorname{Ext}_{\left(Q_{0}, S\right)}^{k}\left(\rho_{3}, \rho_{3}\right)=0
$$

for all $k \in \mathbb{Z} \backslash\{0\}$.

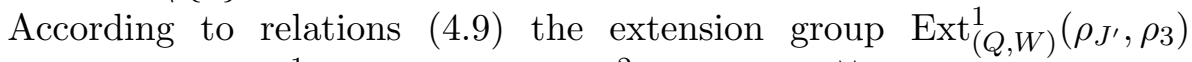
decomposes into $\operatorname{Ext}_{\left(Q_{0}, S\right)}^{1}\left(\rho_{J^{\prime}}, \rho_{3}\right) \oplus \operatorname{Ext}_{\left(Q_{0}, S\right)}^{2}\left(\rho_{3}, \rho_{J^{\prime}}\right)^{\vee}$. Since we have just proved the second summand vanishes, it follows any extension of the form (4.11) must be mapped by tilting to a representation $\rho_{F}$ of $\left(Q_{0}, S\right)$.

Since $\rho_{F}$ is an extension of $\rho_{J^{\prime}}$ by $W \otimes \rho_{3}$, the extension group $\operatorname{Ext}_{\left(Q_{0}, S\right)}^{2}$ $\left(\rho_{F}, \rho_{F}\right)$ is computed by the complex $\operatorname{Ext}^{*}\left(\rho_{J} \oplus \rho_{3}, \rho_{J} \oplus W \otimes \rho_{3}\right)$ with the differential $\delta^{x}$ where $x$ is the Maurer-Cartan element corresponding to the extension class in $\operatorname{Ext}_{\left(Q_{0}, S\right)}^{1}\left(\rho_{J^{\prime}}, W \otimes \rho_{3}\right)$. The vanishing results $(4.14),(4.15)$ imply that $\operatorname{Ext}_{\left(Q_{0}, S\right)}^{2}\left(\rho_{F}, \rho_{F}\right)$ is isomorphic to the cokernel of the map

$$
\operatorname{Ext}_{\left(Q_{0}, S\right)}^{1}\left(\rho_{J^{\prime}}, \rho_{J^{\prime}}\right) \oplus \operatorname{Ext}_{\left(Q_{0}, S\right)}^{1}\left(\rho_{J^{\prime}}, W \otimes \rho_{3}\right) \stackrel{\delta^{x}}{\longrightarrow} \operatorname{Ext}_{\left(Q_{0}, S\right)}^{2}\left(\rho_{J^{\prime}}, W \otimes \rho_{3}\right)
$$

Because $x \in \operatorname{Ext}_{\left(Q_{0}, S\right)}^{1}\left(\rho_{J^{\prime}}, W \otimes \rho_{3}\right)$, the above morphism simplifies to

$$
\operatorname{Ext}_{\left(Q_{0}, S\right)}^{1}\left(\rho_{J^{\prime}}, \rho_{J^{\prime}}\right) \stackrel{\delta^{x}}{\longrightarrow} \operatorname{Ext}_{\left(Q_{0}, S\right)}^{2}\left(\rho_{J^{\prime}}, W \otimes \rho_{3}\right)
$$

Vanishing of $\operatorname{Ext}_{\left(Q_{0}, S\right)}^{2}\left(\rho_{F}, \rho_{F}\right)$ is equivalent with the above morphism being surjective. Furthermore, relations (4.9) and the vanishing results (4.14) imply that

$$
\begin{aligned}
\operatorname{Ext}_{\left(Q_{0}, S\right)}^{1}\left(\rho_{J^{\prime}}, \rho_{J^{\prime}}\right) & =\operatorname{Ext}_{(Q, W)}^{1}\left(\rho_{J^{\prime}}, \rho_{J^{\prime}}\right), \\
\operatorname{Ext}_{\left(Q_{0}, S\right)}^{2}\left(\rho_{J^{\prime}}, W \otimes \rho_{3}\right) & =\operatorname{Ext}_{(Q, W)}^{2}\left(\rho_{J^{\prime}}, W \otimes \rho_{3}\right) .
\end{aligned}
$$

Then derived equivalence with $D^{b}\left(Z^{+}\right)$maps the morphism (4.16) to the connecting morphism

$$
\operatorname{Ext}_{Z^{+}}^{1}\left(J^{\prime}, J^{\prime}\right) \stackrel{\delta}{\longrightarrow} \operatorname{Ext}_{Z^{+}}^{2}\left(J^{\prime}, W \otimes \mathcal{O}_{C_{0}^{+}(-1)}\right)
$$


In order to show that $\delta$ is a surjection recall that according to Lemma 2.6 there are isomorphisms

$$
\varphi_{k}: \operatorname{Ext}_{Z^{+}}^{k}\left(J^{\prime}, W \otimes \mathcal{O}_{C_{0}^{+}}(-1)\right) \stackrel{\sim}{\longrightarrow} \operatorname{Ext}_{D^{+}}^{k-1}\left(J, W \otimes \mathcal{O}_{p}\right)
$$

Moreover, Corollary 2.7 shows that an extension $e \in \operatorname{Ext}_{Z^{+}}^{1}\left(J^{\prime}, W \otimes\right.$ $\left.\mathcal{O}_{C_{0}^{+}}(-1)\right)$ is nondegenerate if and only if the corresponding morphism $\varphi_{1}(e)$ is surjective. In particular, this holds for the extension class $e^{x}$ corresponding to the Maurer-Cartan element $x$. Let $\psi=\varphi_{1}\left(e_{x}\right)$ and $\psi_{*}: \operatorname{Ext}_{D^{+}}^{1}\left(J^{\prime}, J^{\prime}\right) \rightarrow$ $\operatorname{Ext}_{D^{+}}^{1}\left(J^{\prime}, W \otimes \mathcal{O}_{p}\right)$ the natural induced morphism of extensions. Clearly the following diagram commutes.

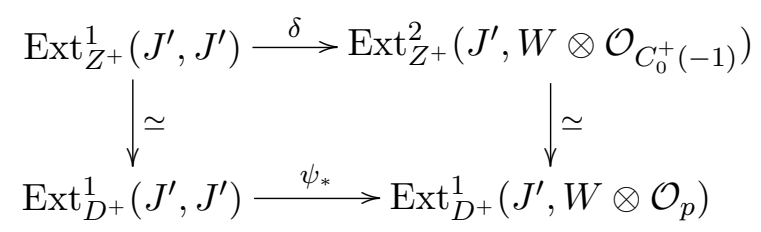

Since $\psi$ is surjective, surjectivity of $\psi_{*}$ follows from the vanishing result $\operatorname{Ext}_{D}^{2}\left(J^{\prime}, \operatorname{Ker}(\psi)\right)=0$ obtained in the proof of Lemma 3.9, and Remark 3.10.

\subsection{Motivic weights in local model}

Next it will be shown that Lemma 4.3 yields a presentation of the motivic weights $w_{F}$ as motivic Milnor fibers of polynomial functions. Note that the quiver $Q$ in (4.6) is the $\operatorname{Ext}^{1}$ quiver associated to four spherical objects $S_{i}, i=1, \ldots, 4$ in the derived category $D^{b}\left(Z^{+}\right)$. Moreover the objects $S_{i}$, $i=1, \ldots, 4$ generate the subcategory consisting of complexes with topological support on $D^{+} \cup C_{0}^{+}$. The images of these objects via the tilting functor generate the subcategory of complexes of quiver representations with nilpotent cohomology. In particular, the representation $\rho_{F}$ corresponding to a sheaf $F$ as in Lemma 4.3 is obtained by successive extensions of the $S_{i}$, $i=1, \ldots, 4$.

For a dimension vector $v=\left(v_{i}\right)_{1 \leq i \leq 4}$, let $\mathbb{A}(v)$ denote the affine space parameterizing all representations of the quiver $Q$ without relations. Note that there is an obvious direct sum decomposition

$$
\mathbb{A}(v)=\mathbb{A}^{r}(v) \oplus \mathbb{A}^{l}(v),
$$


where $\mathbb{A}^{r}(v), \mathbb{A}^{l}(v)$ denote the linear subspaces associated to the right directed, and left directed arrows respectively in diagram (4.6). There is also a natural $\mathbb{G}(v)=\prod_{i=1}^{4} G L\left(v_{i}\right)$ action on $\mathbb{A}(v)$.

The potential (4.7) determines a $\mathbb{G}(v)$-invariant quartic polynomial function $\mathcal{W}$ on $\mathbb{A}(v)$ such that quiver representations of dimension vector $v=$ $\left(v_{i}\right)_{1 \leq i \leq 4}$ are in one-to-one correspondence with closed points in the critical locus $\operatorname{Crit}(\mathcal{W})$.

Let $\rho_{F} \in \mathbb{A}(v)$ be a closed point corresponding to a sheaf $F$ satisfying the conditions of Lemma 4.3. Let $\mathcal{W}_{\rho_{F}}$ be the Taylor series expansion of $\mathcal{W}$ at $\rho_{F}$. Since $\rho_{F}$ is an iterated extension of the spherical objects $S_{i}, i=1, \ldots, 4$, the computation of $w_{\rho_{F}}=w_{F}$ will be carried out in close analogy with the proof of [26, Thm. 8, Section 6.3].

Suppose $E_{1}, E_{2}$ are any two objects in derived category of quiver representations with nilpotent cohomology. Let $E_{0}=E_{1} \oplus E_{2}$. Suppose moreover that the potential function $W_{E_{0}}$ on

$$
\begin{aligned}
\operatorname{Ext}^{1}\left(E_{0}, E_{0}\right)= & \operatorname{Ext}^{1}\left(E_{1}, E_{1}\right) \oplus \operatorname{Ext}^{1}\left(E_{2}, E_{1}\right) \oplus \operatorname{Ext}^{1}\left(E_{1}, E_{2}\right) \\
& \oplus \operatorname{Ext}^{1}\left(E_{2}, E_{2}\right)
\end{aligned}
$$

is minimal i.e., has no quadratic part. Let $\alpha \in \operatorname{Hom}\left(E_{2}[-1], E_{1}\right)$ be a nontrivial element, and let $E_{\alpha}=\operatorname{Cone}(\alpha)$. As in Step 3 in the proof of [26, Thm. 8, Section 6.3], let $W_{(0, \alpha, 0,0)}$ denote the Taylor expansion of $W_{E_{0}}$ at the point $(0, \alpha, 0,0)$ in $\operatorname{Ext}^{1}\left(E_{0}, E_{0}\right)$. Then $W_{(0, \alpha, 0,0)}$ is related by a formal change of variables to a direct sum of the form

$$
W_{E_{\alpha}}^{\min } \oplus \widetilde{Q}_{E_{\alpha}} \oplus \widetilde{N}_{E_{\alpha}}
$$

where $\widetilde{Q}_{E_{\alpha}}$ is a nondegenerate quadratic form and $\widetilde{N}_{E_{\alpha}}$ the zero function on a linear subspace. This implies that there is an identity

$$
\left(1-M F_{0}\left(W_{(0, \alpha, 0,0)}\right)\right)=\left(1-M F_{0}\left(W_{E_{\alpha}}^{\min }\right)\left(1-M F_{0}\left(\widetilde{Q}_{E_{\alpha}}\right)\right) .\right.
$$

Note that $\widetilde{Q}_{E_{\alpha}}$ is not the same as the intrinsic quadratic form $Q_{E_{\alpha}}$. In fact, the discrepancy between these two forms leads to the need to introduce orientation data in order to obtain a well defined integration map.

Two identities for the quadratic form $\widetilde{Q}_{E_{\alpha}}$ follow from the proof of [26, Thm. 8, Section 6.3]. First, the rank of $\widetilde{Q}_{E_{\alpha}}$ is expressed in terms of dimensions of Ext groups as follows:

$$
\operatorname{rk}\left(\widetilde{Q}_{E_{\alpha}}\right)=\left(E_{\alpha}, E_{\alpha}\right)_{\leq 1}-\left(E_{0}, E_{0}\right)_{\leq 1} .
$$


Next, there is a cocycle identity for motivic Milnor fibers above [26, Def. 18, Section 6.3] which reads

$$
\begin{aligned}
& \mathbb{L}^{-\operatorname{rk}\left(Q_{E_{\alpha}}\right) / 2}\left(1-M F_{0}\left(Q_{E_{\alpha}}\right)\right) \\
& =\mathbb{L}^{-\operatorname{rk}\left(\widetilde{Q}_{E_{\alpha}}\right) / 2}\left(1-M F_{0}\left(\widetilde{Q}_{E_{\alpha}}\right)\right) \prod_{i=1}^{2} \mathbb{L}^{-\operatorname{rk}\left(Q_{E_{i}}\right) / 2}\left(1-M F_{0}\left(Q_{E_{i}}\right)\right) .
\end{aligned}
$$

In the present case, $E_{0}$ is a direct sum of simple objects

$$
E_{0}=\bigoplus_{i=1}^{4} S_{i}^{\oplus v_{F}(i)}
$$

where $v_{F}=\left(v_{F}(i)\right)_{1 \leq i \leq 4}$ is the dimension vector of the extension $\rho_{F}$ of Lemma 4.2 ,

$$
v_{H}=((N+2) k-n,(N+1) k-n, N k-n+r, N k-n) .
$$

Then Equation (4.17) yields

$$
1-M F_{0}\left(\mathcal{W}_{\rho_{F}}\right)=\left(1-M F_{0}\left(W_{F}^{\min }\right)\right)\left(1-M F_{0}\left(\widetilde{Q}_{\rho_{F}}\right)\right) .
$$

where $\widetilde{Q}_{\rho_{F}}$ is a quadratic form which satisfies two identities analogous to (4.18), (4.19). Therefore, the rank of $\widetilde{Q}_{\rho_{F}}$ is given by

$$
\begin{aligned}
\operatorname{rk}\left(\widetilde{Q}_{\rho_{F}}\right) & =(F, F)_{\leq 1}-\left(E_{0}, E_{0}\right)_{\leq 1} \\
& =(F, F)_{\leq 1}+\operatorname{dim}\left(\mathbb{A}\left(v_{F}\right)\right)-\operatorname{dim}\left(\mathbb{G}\left(v_{F}\right)\right) .
\end{aligned}
$$

Moreover there is a cocycle identity

$$
\mathbb{L}^{-\operatorname{rk}\left(\widetilde{Q}_{\rho_{F}}\right) / 2}\left(1-M F_{0}\left(\widetilde{Q}_{\rho_{F}}\right)\right)=\mathbb{L}^{-\operatorname{rk}\left(Q_{F}\right) / 2}\left(1-M F_{0}\left(Q_{F}\right)\right)
$$

since $Q_{S_{i}}=0, i=1, \ldots, 4$ for the spherical objects. Equations (4.20), (4.21), (4.22) then yield the following expression:

$$
w_{F}=\mathbb{L}^{\left(\operatorname{dim}\left(\mathbb{G}\left(v_{F}\right)\right)-\operatorname{dim}\left(\mathbb{A}\left(v_{F}\right)\right) / 2\right.}\left(1-M F_{0}\left(\mathcal{W}_{\rho_{F}}\right)\right),
$$

where $\mathcal{W}_{\rho_{F}}$ is the polynomial function

$$
\mathcal{W}_{\rho_{F}}(\rho)=\mathcal{W}\left(\rho+\rho_{F}\right)
$$

for any $\rho \in \mathbb{A}\left(v_{F}\right)$. Note that $M F_{0}\left(\mathcal{W}_{\rho_{F}}\right)=M F_{\rho_{F}}(\mathcal{W})$ by functoriality of motivic Milnor fibers. 
In general explicit computations of pointwise Milnor fibers are difficult. The following Lemma shows that the computation is tractable on a certain subset of the critical locus of $\mathcal{W}$. Let $M C_{0}=\operatorname{Crit}(\mathcal{W}) \cap \mathbb{A}^{r}(v)$ be the subscheme of critical points with trivial left directed arrows. The potential $\mathcal{W}: \mathbb{A}(v) \rightarrow \mathbb{C}$ is of the form

$$
\mathcal{W}=\sum_{\kappa=1}^{K} y_{\kappa} P_{\kappa}
$$

where $\left(y_{\kappa}\right)_{1 \leq \kappa \leq K}$ are natural linear coordinates on $\mathbb{A}^{l}(v)$ and $P_{\kappa}: \mathbb{A}^{r}(v) \rightarrow \mathbb{C}$ are polynomial functions. Then $M C_{0}$ is determined by

$$
y_{\kappa}=0, \quad P_{\kappa}=0, \quad \kappa=1, \ldots, K \text {. }
$$

Let $\mathcal{X}_{0}=\mathcal{W}^{-1}(0)$ denote the central fiber. Note that there is a commutative diagram

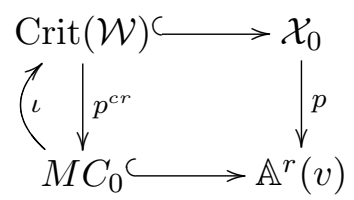

where $p: \mathcal{X}_{0} \rightarrow \mathbb{A}^{r}(v)$ is the restriction of the canonical projection $\mathbb{A}(v) \rightarrow$ $\mathbb{A}^{r}(v)$ and $\iota$ is the zero section $y_{\kappa}=0, \kappa=1, \ldots, K$. Note that the fibers of $p, p^{c r}$ are linear subspaces of $\mathbb{A}^{l}(v)$. Let $M C_{0}^{s m}$ denote the smooth open locus of $M C_{0}$.

Lemma 4.4. Let $\rho \in M C_{0}^{s m}$. Then the motivic weight at $\iota(\rho)$ is

$$
1-M F_{\iota(\rho)}(\mathcal{W})=\mathbb{L}^{\operatorname{dim} \mathbb{A}^{l}(v)} .
$$

Proof. Let $\mathcal{U} \subset \mathbb{A}(v)$ be the open subset where the Jacobian matrix of the polynomial functions $\left(P_{\kappa}\right), \kappa=1, \ldots, K$ has maximal rank. Then $\mathcal{U} \cap$ $M C_{0}=M C_{0}^{s m}$. Let $\mathcal{Y}_{0}$ be the restriction of the central fiber $\mathcal{X}_{0}$ to $\mathcal{U}$ and $q: \mathcal{Y}_{0} \rightarrow \mathbb{A}^{r}(v)$ the restriction of $p$. Note that the singular locus $\mathcal{Y}_{0}^{\text {sing }} \subset \mathcal{Y}_{0}$ is determined by the equations

$$
y_{\kappa}=0, \quad P_{\kappa}=0, \quad \kappa=1, \ldots, K
$$

This follows from the fact that there is a factorization

$$
\mathcal{U} \stackrel{P}{\longrightarrow} \mathbb{A}^{l}(v) \times \mathbb{A}^{l}(v) \stackrel{Q}{\longrightarrow} \mathbb{C}
$$


of $\left.\mathcal{W}\right|_{\mathcal{U}}: \mathcal{U} \rightarrow \mathbb{C}$, where

$$
P\left(y_{\kappa}, x\right)=\left(y_{\kappa}, P_{\kappa}(x)\right)
$$

for any $x \in \mathbb{A}^{r}(v),\left(y_{\kappa}\right) \in \mathbb{A}^{l}(v)$ and

$$
Q\left(y_{\kappa}, z_{\kappa}\right)=\sum_{\kappa=1}^{K} y_{\kappa} z_{\kappa}
$$

Since the Jacobian matrix of $\left(P_{\kappa}\right)$ has maximal rank on $\mathcal{U}$, the map $P$ is smooth. Moreover, the singular locus of the central fiber of $Q$ is obviously $y_{\kappa}=z_{\kappa}=0$ for all $\kappa=1, \ldots, K$. This implies the claim.

In conclusion, $\mathcal{Y}_{0}^{\text {sing }}$ coincides with the image $\iota\left(M C_{0}^{s m}\right) \subset \mathcal{Y}_{0}$. Note also that the fibers of $p$ over closed points $\rho \in M C_{0}^{s m}$ are isomorphic to $\mathbb{A}^{l}(v)$. Then a normal crossing resolution of $\mathcal{Y}_{0}$ can be obtained by a single embedded blow-up. Let $\sigma: \mathcal{U}^{\prime} \rightarrow \mathcal{U}$ be the blow-up of $\mathcal{U}$ along the linear subspace

$$
y_{\kappa}=0, \quad \kappa=1, \ldots, K
$$

The total transform $\sigma^{-1}\left(\mathcal{Y}_{0}\right)$ consists of the strict transform $\mathcal{Y}_{0}^{\prime}$ and an exceptional divisor $D$ isomorphic to a $\mathbb{P}\left(\mathbb{A}^{l}(v)\right)$-bundle over $\mathbb{A}^{r}(v)$. The strict transform $\mathcal{Y}_{0}^{\prime}$ is smooth and intersects $D$ transversely along a divisor $D^{\prime} \subset$ $\mathcal{Y}_{0}^{\prime}$, which is isomorphic to a $\mathbb{P}\left(\mathbb{A}^{l}(v)\right.$ )-bundle over $M C_{0}^{s m}$. Moreover, both $\mathcal{Y}_{0}^{\prime}$ and $D$ multiplicity 1 in $\sigma^{-1}\left(\mathcal{Y}_{0}\right)$.

For any point $\rho \in M C_{0}^{s m}, \sigma^{-1}(\iota(\rho))$ intersects both $\mathcal{Y}_{0}^{\prime}$ and $D$ along the fiber $D_{\rho} \subset D$, which is isomorphic to $\mathbb{P}\left(\mathbb{A}^{l}(v)\right)$. Therefore, from the definition [26, Section 4, p. 67]

$$
1-M F_{\iota(\rho)}(\mathcal{W})=1-(1-\mathbb{L})\left[\mathbb{P}\left(\mathbb{A}^{l}(v)\right)\right]=\mathbb{L}^{\operatorname{dim}\left(\mathbb{A}^{l}(v)\right)} .
$$

\subsection{Comparison with refined Hilbert scheme invariants}

The compact motivic version of Hilbert scheme invariants has been defined in Equation (1.11), which is reproduced below for convenience

$$
Z_{C}^{\mathrm{mot}}(q, a)=\sum_{l, r \geq 0} q^{2 l} a^{2 r} \mathbb{L}^{r^{2} / 2}\left[H^{[l, r]}(C)\right] .
$$

Note that the Chow motive of the nested Hilbert $\left[H^{[l, r]}(C)\right]$ is equal to the Chow motive $\left[Q^{[l, r]}(C)\right]$ of the relative Quot scheme defined above Proposition 3.6. Moreover, the stack $\mathcal{Q}(X, C, r, n)$ is a $\mathbb{C}^{\times}$gerbe over the relative Quot scheme $Q^{[l, r]}(C), l=n-\chi\left(\mathcal{O}_{C}\right)$, according to Proposition 3.6. 
As observed in Remark 3.8, the moduli stack $\mathcal{M}(X, C, r, n)$ is also a $\mathbb{C}^{\times}$ gerbe over a coarse moduli scheme $M^{[l, r]}(C)$, and there is a natural forgetful morphism $\pi: Q^{[l, r]}(C) \rightarrow M^{[l, r]}(C)$. Note also that there is a natural stratification of $M^{[l, r]}(C)$ such that the restriction of $\pi$ to each stratum is a smooth projective bundle with fiber $\mathbb{P}^{h^{0}(F)-1}$. Since the motivic weights $w_{F}$ are invariant under isomorphisms, $F \simeq F^{\prime}$, they descend to motivic weights $w_{[F]}$ on the coarse moduli space $M^{[l, r]}(C)$.

Then using the conjectural identity (4.5) a stratification argument implies that the virtual motive of the stack function $f: \mathcal{Q}(X, C, r, n) \hookrightarrow$ $O b(\mathcal{A})$ is given by

$$
\frac{1}{\mathbb{L}-1} \Phi([f: \mathcal{Q}(X, C, r, n) \rightarrow O b(\mathcal{A})])=\mathbb{L}^{(1-n) / 2} \int_{M^{[l, r]}(C)}\left[\mathbb{P}^{h^{0}(F)-1}\right] w_{[F]} .
$$

Applying Lemmas 4.4, 4.3, one then obtains

$$
\begin{aligned}
& \frac{1}{\mathbb{L}-1} \Phi([f: \mathcal{Q}(X, C, r, n) \rightarrow O b(\mathcal{A})]) \\
& \quad=\mathbb{L}^{(1-n) / 2} \mathbb{L}^{\left(\operatorname{dim}\left(\mathbb{G}\left(v_{F}\right)\right)-\operatorname{dim}\left(\mathbb{A}\left(v_{F}\right)\right) / 2+\operatorname{dim}\left(\mathbb{A}^{l}\left(v_{F}\right)\right) / 2\right.} \int_{M^{[l, r]}(C)}\left[\mathbb{P}^{h^{0}(F)-1}\right]
\end{aligned}
$$

Note that

$$
\left(\operatorname{dim}\left(\mathbb{G}\left(v_{F}\right)\right)-\operatorname{dim}\left(\mathbb{A}\left(v_{F}\right)\right) / 2+\operatorname{dim}\left(\mathbb{A}^{l}\left(v_{F}\right)\right) / 2=\left(r^{2}-k^{2}\right) / 2\right.
$$

by a straightforward computation. Therefore the final formula is

$$
\frac{1}{\mathbb{L}-1} \Phi([f: \mathcal{Q}(X, C, r, n) \rightarrow O b(\mathcal{A})])=\mathbb{L}^{\left(r^{2}-k^{2}+1-n\right) / 2}\left[Q^{[l, r]}(C)\right] .
$$

Then the resulting generating function of $C$-framed virtual motivic invariants in the small $b>0$ chamber is

$$
\begin{aligned}
Z_{0+}^{\operatorname{mot}}(X, C ; u, T) & =\mathbb{L}^{\left(1-k^{2}\right) / 2} \sum_{r \geq 0} \sum_{l \geq 0} u^{n} T^{r} \mathbb{L}^{\left(r^{2}-n\right) / 2}\left[Q^{[l, r]}(C)\right] \\
& =\mathbb{L}^{\left(1-k^{2}-\chi\left(\mathcal{O}_{C}\right)\right) / 2} u^{\chi\left(\mathcal{O}_{C}\right)} \sum_{r \geq 0} \sum_{l \geq 0} u^{l} T^{r} \mathbb{L}^{\left(r^{2}-l\right) / 2}\left[Q^{[l, r]}(C)\right]
\end{aligned}
$$

In conclusion, note that identity (1.16) holds i.e.,

$$
Z_{0+}^{\operatorname{mot}}\left(X, C ; q^{2} \mathbb{L}^{1 / 2}, a^{2}\right)=\mathbb{L}^{\left(1-k^{2}\right) / 2} q^{2 \chi\left(\mathcal{O}_{C}\right)} Z_{C}^{\operatorname{mot}}(q, a) .
$$




\section{Acknowledgments}

We would like to thank Sergei Gukov, Sheldon Katz, Melissa Liu, Davesh Maulik, Kentaro Nagao, Alexei Oblomkov, Andrei Okounkov, Rahul Pandharipande, Vivek Shende, Richard Thomas, and Cumrun Vafa for very helpful discussions. D.-E.D is very grateful to Alexei Oblomkov, Vivek Shende and Cumrun Vafa for sharing their ideas and insights during collaboration on related projects. D.-E.D. would also like to acknowledge the partial support of the Moduli Space Program 2011 at Isaac Newton Institute, the Simons Workshop on Mathematics and Physics 2011, the Simons Center for Geometry and Physics, and NSF grant PHY-0854757-2009 during completion of this work. Y.S. thanks IHES for excellent research conditions. His work was partially supported by NSF grant DMS-1101554.

\section{Appendix A. Wall-crossing formula}

For completeness, a proof of Proposition 1.2 is presented here in detail. In the view of Theorem 1.3, this proves Theorem 1.1. Although the motivic Donaldson-Thomas theory of [26] is consistently used throughout this paper, the proof of Equation (1.14) will be based on the alternative wall-crossing formalism developed in [20-24]. The first is more general, but requires more work on the foundations, as explained in detail in Section 4. As stated in the main text, several similar computations have already been carried out in the literature, for example in [50, Section 4.3] and [40, Thm 3.15], and also [8, Section 2], [6, Section 3]. The approach explained below follows closely [8, Section 2], [6, Section 3]. For clarity the proof will be structured in several steps, and a brief review of motivic Hall algebras will be provided in the process.

\section{A.1. Critical stability parameters}

In the framework of Section 2.2 fix a polarization $\omega$ of $X$ such that $\int_{C_{0}} \omega=1$. Recall that $b_{c} \in \mathbb{R}$ is called critical of type $(r, n) \in \mathbb{Z}_{\geq 0} \times \mathbb{Z}$ if there exist strictly $\mu_{(\omega, B)}$-semistable $C$-framed coherent sheaves $E$ with numerical invariants $\operatorname{ch}(E)=\left(-1,0,[C]+r\left[C_{0}\right], n\right)$.

First note the following consequence of the defining conditions $(C .1)$, (C.2) for the subcategory $\mathcal{A}^{C} \subset \mathcal{A}$ in Section 2.2 
Lemma A.1. Let $E$ be a $C$-framed perverse coherent sheaf with $\operatorname{ch}(E)=$ $\left(-1,0,[C]+r\left[C_{0}\right], n\right), r \in \mathbb{Z}_{>0}$. Let $F \subset E$ and $E \rightarrow G$ be a nontrivial subobject, respectively quotient of $E$ in $\mathcal{A}^{C}$, where $F, G$ are pure dimension one sheaves. Then $F, G$ are topologically supported on $C_{0}$ and $\operatorname{ch}_{2}(F)=r_{F}\left[C_{0}\right]$, $\operatorname{ch}_{2}(G)=r_{G}\left[C_{0}\right]$ for some integers $0<r_{F}, r_{G} \leq r$.

Proof. It suffices to prove one case, since the other is analogous. Suppose $E \rightarrow G$ is a pure dimension one quotient in $\mathcal{A}^{C}$ and let $E^{\prime}=\operatorname{Ker}(E \rightarrow G)$. Since $\operatorname{ch}(E)=\left(-1,0,[C]+r\left[C_{0}\right]\right)$ and $G$ is pure dimension one, $\operatorname{ch}_{0}\left(E^{\prime}\right)=$ $-1, \operatorname{ch}_{1}\left(E^{\prime}\right)=0$. The second defining condition (C.2) of $\mathcal{A}^{C}$ in Section 2.2 implies that $G$ must be topologically supported on $C_{0}$. Therefore, $\operatorname{ch}_{2}(G)=$ $r_{G}\left[C_{0}\right], r_{G} \in \mathbb{Z}_{>0}$ and $\operatorname{ch}_{0}\left(E^{\prime}\right)=\left(-1,0,[C]+\left(r-r_{G}\right)\left[C_{0}\right], n^{\prime}\right)$. Moreover, the first defining condition $(C .1)$ implies that $\mathcal{H}^{-1}\left(E^{\prime}\right)$ must be the ideal sheaf of a closed subscheme $Z_{E^{\prime}} \subset X$, which according to $(C .2)$ must be topologically supported on the union $C \cup C_{0}$. Since $(C .2)$ also requires $\mathcal{H}^{0}(E)$ to be topologically supported on $C \cup C_{0}$, it follows that $r-r_{G} \geq 0$.

Lemma A.2. Let $b_{c} \in \mathbb{R}$ be a critical stability parameter of type $(r, n)$ and $E$ a strictly $\mu_{\left(\omega, b_{c}\right)}$-semistable object of $\mathcal{A}^{C}$ with $\operatorname{ch}(E)=(-1,0,[C]+$ $\left.r\left[C_{0}\right], n\right)$. Then one of the following two cases holds.

(i) There is an exact sequence

$$
0 \rightarrow E^{\prime} \rightarrow E \rightarrow G \rightarrow 0
$$

in $\mathcal{A}_{1 / 2}^{C}$, where $G$ is an $\omega$-slope semistable pure dimension one sheaf set theoretically supported on $C_{0}$ with $\mu_{\omega}(G)=-2 b_{c}$. Moreover, $\operatorname{ch}_{2}(G)=r_{G}\left[C_{0}\right]$ with $0<r_{G} \leq r$.

(ii) There is an exact sequence

$$
0 \rightarrow F \rightarrow E \rightarrow E^{\prime \prime} \rightarrow 0
$$

in $\mathcal{A}_{1 / 2}^{C}$, where $F$ is an $\omega$-slope semistable pure dimension one sheaf set theoretically supported on $C_{0}$ with $\mu_{\omega}(F)=-2 b_{c}$. Moreover, $\operatorname{ch}_{2}(F)=r_{F}\left[C_{0}\right]$ with $0<r_{F} \leq r$.

Proof. This lemma follows from the stability criterion 2.1 applied to $C$-framed perverse coherent sheaves. By definition, if $E$ is strictly $\mu_{\left(\omega, b_{c}\right)^{-}}$ semistable, one of the following two cases must hold. 
(a) There is a strict epimorphism $E \rightarrow G$ in $\mathcal{A}_{1 / 2}^{C}$ with $G$ a nontrivial pure dimension one sheaf on $X$ such that

$$
\mu_{\left(\omega, b_{c}\right)}(G)=-3 b_{c}
$$

(b) There is an $\mu_{(\omega, B)}$-semistable $C$-framed perverse coherent sheaf $E$ of type $(r, n)$ and a strict monomorphism $F \hookrightarrow$ in $\mathcal{A}_{1 / 2}^{C}$ with $F$ a pure dimension one sheaf on $X$ such that

$$
\mu_{\left(\omega, b_{c}\right)}(F)=-3 b_{c}
$$

Suppose $(a)$ holds. According to Lemma A.1, $G$ must be topologically supported on $C_{0}$ and $\operatorname{ch}_{2}(G)=r_{G}\left[C_{0}\right]$ with $0<r_{G} \leq r$. Suppose $G \rightarrow G^{\prime}$ is a nontrivial pure dimension one quotient and let $K \subset E$ be the kernel of the resulting surjective morphism $E \rightarrow G^{\prime \prime}$ in $\mathcal{A}^{C}$. Then $K$ must belong to $\mathcal{A}_{1 / 2}^{C}$ since $E$ does, hence $E \rightarrow G^{\prime \prime}$ is a strict epimorphism. If $\mu_{\left(\omega, b_{c}\right)}\left(G^{\prime \prime}\right)<-3 b_{c}$ this quotient destabilizes $E$, leading to a contradiction. Therefore $\mu_{\left(\omega, b_{c}\right)}\left(G^{\prime \prime}\right) \geq-3 b_{c}=\mu_{\left(\omega, b_{c}\right)}(G)$, which proves that $G$ is $\omega$-slope semistable. This leads to case $(i)$ in Lemma A.2.

Case (b) leads analogously to case $(i i)$.

Corollary A.3. Under the conditions of Lemma A.2, there exist $b_{-}, b_{+} \in \mathbb{R}$ with $b_{-}<b_{c}<b_{+}$such that $b_{c}$ is the only critical stability parameter of type $(r, n)$ in the interval $\left[b_{-}, b_{+}\right]$.

Proof. Choose some $b_{-}<b_{c}<b_{+}$. Lemma A.2 implies that any critical stability parameter $b_{c}^{\prime}$ must be of the form

$$
b_{c}^{\prime}=-\frac{n^{\prime}}{2 r^{\prime}}
$$

with $n^{\prime}, r^{\prime} \in \mathbb{Z}, 1 \leq r^{\prime} \leq r$. Therefore, the set of stability parameters in the interval $\left[b_{-}, b_{+}\right]$is a subset of the set of integers $n^{\prime}$ satisfying

$$
-2 r\left|b_{+}\right| \leq n^{\prime} \leq 2 r\left|b_{-}\right|
$$

The latter is a finite set for fixed $b_{-}, b_{+}, r$. Therefore, there exist $b_{-}^{\prime}<b_{c}<b_{+}^{\prime}$ sufficiently close to $b_{c}$ such that there are no critical stability parameters of type $(r, n)$ in the interval $\left[b_{-}^{\prime}, b_{+}^{\prime}\right]$. 
Lemma A.4. Suppose $b_{c}$ is a critical stability parameter of type $(r, n) \in$ $\mathbb{Z}_{\geq 1} \times \mathbb{Z}$. Then there exist two constants $\epsilon_{+}, \epsilon_{-}$, such that the following holds for any stability parameters

$$
b_{c}-\epsilon_{-}<b_{-}<b_{c}<b_{+}<b_{c}+\epsilon_{+} .
$$

Suppose $E$ is a $\mu_{\left(\omega, b_{c}\right)}$-semistable $C$-framed perverse coherent sheaf with $\operatorname{ch}(E)=\left(-1,0,[C]+r\left[C_{0}\right], n\right)$. Then

(i) $E$ is either $\mu_{\left(\omega, b_{+}\right)}$-semistable or has a Harder-Narasimhan filtration

$$
0 \subset E^{\prime} \subset E
$$

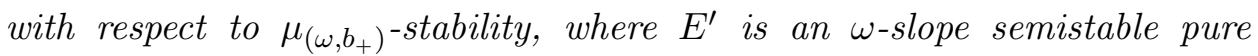
dimension one sheaf with topological support on $C_{0}$ and $\mu_{\omega}\left(E^{\prime}\right)=-2 b_{c}$. The quotient $E^{\prime \prime}=E / E^{\prime}$ is an $\mu_{\left(\omega, b_{+}\right)}$-semistable $C$-framed perverse coherent sheaf.

(ii) E is either $\mu_{\left(\omega, b_{-}\right)}$-semistable or has a Harder-Narasimhan filtration

$$
0 \subset E^{\prime} \subset E
$$

with respect to $\mu_{\left(\omega, b_{-}\right)}$-stability, where $E^{\prime}$ is a $\mu_{\left(\omega, b_{-}\right)}$-semistable $C$-framed perverse coherent sheaf. The quotient $E^{\prime \prime}=E^{\prime} / E$ is an $\omega$-slope semistable pure dimension one sheaf with topological support on $C_{0}$ and $\mu_{\omega}\left(E^{\prime \prime}\right)=-2 b_{c}$.

Proof. It suffices to prove $(i)$, the second statement being entirely analogous. The existence of a Harder-Narasimhan filtration follows from the fact that

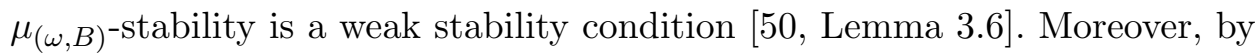
construction all successive quotients of the Harder-Narasimhan filtration of an object of $\mathcal{A}_{1 / 2}^{C}$ also belong to $\mathcal{A}_{1 / 2}^{C}$.

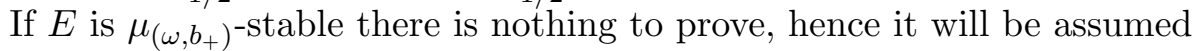
this is not the case. Then let

$$
0=E_{0} \subset E_{1} \subset \cdots \subset E_{h}=E, \quad h \geq 2,
$$

be its Harder-Narasimhan filtration with respect to $\mu_{\left(\omega, b_{+}\right)}$-stability. Let $E \rightarrow E^{\prime \prime}$ be the last quotient of the Harder-Narasimhan filtration. Hence $E^{\prime \prime}$

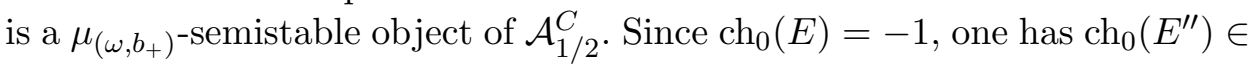
$\{0,1\}$.

Suppose $\operatorname{ch}_{0}\left(E^{\prime \prime}\right)=0$. Then $E^{\prime \prime}$ must be an $\omega$-slope semistable pure dimension one sheaf with topological support on $C_{0}$. Moreover the kernel $E^{\prime}=\operatorname{Ker}\left(E \rightarrow E^{\prime \prime}\right)$ is an object of $\mathcal{A}_{C}^{1 / 2}$ because it admits a filtration such 
that all successive quotients belong to $\mathcal{A}_{1 / 2}^{C}$. Therefore the morphism $E \rightarrow$ $E^{\prime \prime}$ is a strict epimorphism. By the properties of the Harder-Narasimhan filtration,

$$
\mu_{\left(\omega, b_{+}\right)}\left(E^{\prime \prime}\right)<-3 b_{+},
$$

which implies

$$
\mu_{\left(\omega, b_{c}\right)}\left(E^{\prime \prime}\right)<-3 b_{c}
$$

since for any $b \in \mathbb{R}$

$$
\mu_{(\omega, b)}\left(E^{\prime \prime}\right)=\mu_{\omega}\left(E^{\prime \prime}\right)-b .
$$

According to Proposition 2.1, this leads to a contradiction since $E$ is assumed $\mu_{\left(\omega, b_{c}\right)}$-semistable.

In conclusion $\operatorname{ch}_{0}\left(E^{\prime \prime}\right)=-1$. This implies that all other successive quotients, $E_{j} / E_{j-1}, 1 \leq j \leq h-1$, are $\omega$-slope semistable pure dimension one sheaves with topological support on $C_{0}$.

Next note that by construction the induced filtration

$$
0 \subset E_{j} / E_{j-1} \subset \cdots \subset E / E_{j-1}
$$

on each quotient $E / E_{j}, j=1, \ldots, h-1$ is again a Harder-Narasimhan filtration for $\mu_{\left(\omega, b_{+}\right)}$-stability. Therefore,

$$
\mu_{\left(\omega, b_{+}\right)}\left(E_{j+1} / E_{j}\right)>-3 b_{+}
$$

for all $1 \leq j \leq h-1$. At the same time

$$
\mu_{\left(\omega, b_{c}\right)}\left(E_{1}\right) \leq-3 b_{c}
$$

since $E$ is $\left(\omega, b_{c}\right)$-semistable. Then, using the standard properties of HarderNarasimhan filtrations, one obtains

$$
-2 b_{+}<\mu_{\omega}\left(E_{h-1} / E_{h-2}\right)<\cdots<\mu_{\omega}\left(E_{1}\right) \leq-2 b_{c}
$$

However, since all $E_{j} / E_{j-1}, 1 \leq j \leq h-1$ are nontrivial pure dimension sheaves with topological support on $C_{0}$,

$$
\operatorname{ch}_{2}\left(E_{j} / E_{j-1}\right)=r_{j}\left[C_{0}\right]
$$

for some integers $0<r_{j} \leq r$, and

$$
\mu_{\omega}\left(E_{j} / E_{j-1}\right)=\frac{\chi\left(E_{j} / E_{j-1}\right)}{r_{j}} .
$$


Now note that there exists $\epsilon_{+}>0$ sufficiently small such that there are no rational numbers $n^{\prime} / r^{\prime}, 1 \leq r^{\prime} \leq r, n^{\prime} \in \mathbb{Z}$ in the interval $\left(-2 b_{c}-2 \epsilon_{+},-2 b_{c}\right)$. Therefore, if $b_{c}<b_{+}<b_{c}+\epsilon_{+}$inequalities (A.4) imply that $h=2$ and $\mu_{\omega}\left(E_{1}\right)=-2 b_{c}$.

\section{A.2. Motivic Hall algebra identities}

For completeness, recall the construction of the motivic Hall algebra [24, 26] of the perverse coherent sheaf category $\mathcal{A}$. Let $\mathcal{O} b(\mathcal{A})$ be the stack of all objects of $\mathcal{A}$, which is algebraic, locally of finite type over $\mathbb{C}$. A stack function is a pair $(\mathcal{X}, f)$ where $\mathcal{X}$ is an algebraic stack of finite type over $\mathbb{C}$, and $f: \mathcal{X} \rightarrow \mathcal{O} b(\mathcal{A})$ a morphism of algebraic stacks. The underlying vector space of the motivic Hall algebra $H(\mathcal{A})$ is the $\mathbb{Q}$-vector space generated by isomorphism classes of stack functions subject to the relations

$$
[(\mathcal{X}, f)] \sim\left[\left(\mathcal{Y},\left.f\right|_{\mathcal{Y}}\right)\right]+\left[\left(\mathcal{X} \backslash \mathcal{Y},\left.f\right|_{\mathcal{X} \backslash \mathcal{Y}}\right)\right]
$$

for any closed algebraic substack $\mathcal{Y} \subset \mathcal{X}$. The algebra structure is defined by a $\mathbb{Q}$-linear convolution product

$$
\left[\left(\mathcal{X}_{1}, f_{1}\right)\right] *\left[\left(\mathcal{X}_{2}, f_{2}\right)\right]=[(\mathcal{X}, f)],
$$

where $(\mathcal{X}, f)$ is the stack function determined by a diagram of the form

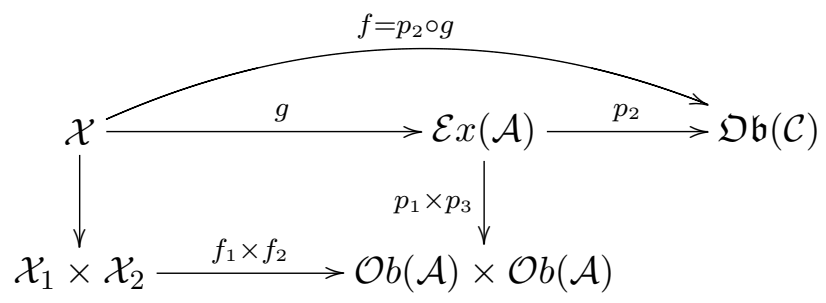

Here $\mathcal{E} x(\mathcal{A})$ denotes the moduli stack of three term exact sequences $0 \rightarrow$ $E_{1} \rightarrow E_{2} \rightarrow E_{3} \rightarrow 0$ in $\mathcal{A}$ and $p_{i}: \mathcal{E} x(\mathcal{A}) \rightarrow \mathcal{O} b(\mathcal{A})$ the three natural forgetful morphisms mapping such a sequence to $E_{i}, 1 \leq i \leq 3$, respectively. The convolution product is associative and has a unit, the stack function $[(\operatorname{Spec}(\mathbb{C}) \rightarrow \mathcal{O} b(\mathcal{A})]$ determined by the zero object of $\mathcal{A}$. Finally, note that the natural commutator $[$,$] of the associative product *$ determines a Poisson algebra structure on $H(\mathcal{A})$.

In the present case, any pair $(r, n) \in \mathbb{Z}_{\geq 0} \times \mathbb{Z}$ and any $b \in \mathbb{R}$, the moduli stack of $\mu_{(\omega, b)}$-semistable $C$-framed perverse coherent sheaves determines an 
element

$$
p_{(\omega, b)}(r, n)=\left[\mathcal{P}_{(\omega, b)}(X, C, r, n) \hookrightarrow O b(\mathcal{A})\right]
$$

of the Hall algebra $H(\mathcal{A})$. Similarly the moduli stack of $\mu_{(\omega, b)}$-stable coherent pure dimension one sheaves $F$ on $X$ with $\operatorname{ch}_{2}(F)=r\left[C_{0}\right]$ and $\chi(F)=n$ determines an element

$$
s_{(\omega, b)}(r, n)=\left[\mathcal{M}_{(\omega, b)}(X, r, n) \hookrightarrow O b(\mathcal{A})\right]
$$

of the same Hall algebra. Since the polarization $\omega$ is fixed throughout this section, while $b$ is varied, the simpler notation $p_{b}(r, n)$ will be used in the following. Similarly, for fixed polarization, $s_{(\omega, b)}(r, n)$ is in fact independent on $b$, hence it will be denoted by $s(r, n)$.

Wall-crossing formulas $[24,26]$ are obtained by converting Lemma A.4 into stack function identities, then applying a suitable integration map. In the construction of [24], the integration map is defined on a certain Poisson subalgebra of $H_{\text {alg }}^{\text {ind }}(\mathcal{A}) \subset H(\mathcal{A})$ which has a complicated technical definition [21, Sect. 5.2]. Omitting the technical details, it suffices to note that the stack function determined by any moduli space of $(\omega, b)$-limit slope semistable stable objects of $\mathcal{A}^{C}$ belongs to $H_{\text {alg }}^{\text {ind }}(\mathcal{A}) \subset H(\mathcal{A})$ as long as there are no strictly semistable objects. In particular, this is the case with the stack functions $p_{b}(r, n)$ for $b \in \mathbb{R}$ non-critical of type $(r, n)$. This fails when strictly semistable objects are present, as is the case with the stack functions $s(r, n)$. In such cases it is proven in [22, Thm. 8.7] that the associated log stack functions

$$
\text { (A.5) } t(r, n)=-\sum_{l \geq 1} \frac{(-1)^{l}}{l} \sum_{\begin{array}{c}
\left(r_{i}, n_{i}\right) \in \mathbb{Z}^{2}, r_{i} \geq 1,1 \leq i \leq l \\
r_{1}+\cdots+r_{l}=r, n_{1}+\cdots+n_{l}=n, \\
n_{i} / r_{i}=n / r, 1 \leq i \leq l
\end{array}} s\left(r_{1}, n_{1}\right) * \cdots * s\left(r_{l}, n_{l}\right)
$$

belong to $H_{\text {alg }}^{i n d}(\mathcal{A})$. The sum in the right-hand side is finite for fixed $(r, n)$ since there is a finite set of decompositions $r=r_{1}+\cdots+r_{l}$ with $r_{i} \geq 1$, $1 \leq i \leq l$.

The integration map

$$
I_{\nu}: H_{\mathrm{alg}}^{\mathrm{ind}}(\mathcal{A}) \rightarrow \Lambda_{\nu}(\mathcal{A})
$$

is a morphism of Poisson algebras determined by a choice of constructible function $\nu$ on the stack of all objects $O b(\mathcal{A})$. It takes values in a Poisson algebra $\Lambda_{\nu}(\mathcal{A})$ spanned over $\mathbb{Q}$ by $\left\{\mathrm{e}_{\alpha}\right\}, \alpha \in K(\mathcal{A})$, where $K(\mathcal{A})$ is the quotient of the Grothendieck group of $\mathcal{A}$ by numerical equivalence, and $\chi\left(\alpha, \alpha^{\prime}\right)$. 
The Poisson bracket is given by

$$
\left[\mathrm{e}_{\alpha}, \mathrm{e}_{\alpha^{\prime}}\right]=(-1)^{\epsilon(\nu) \chi\left(\alpha, \alpha^{\prime}\right)} \chi\left(\alpha, \alpha^{\prime}\right) .
$$

where $\chi\left(\alpha, \alpha^{\prime}\right)$ is the natural antisymmetric bilinear pairing on $K(\mathcal{A})$, and $\epsilon(\nu) \in\{0,1\}$. In principle $\nu$ can be either the constant function $\nu=1$, in which case $\epsilon(\nu)=0$, or Behrend's constructible function, in which case $\epsilon(\nu)=1$. In the present context only the integration map with respect to the constant constructible function $\nu=1$ is rigorously constructed [23, Thm. 6.11]. This yields topological Euler character invariants of objects in $\mathcal{A}^{C}$ defined by

$$
\begin{aligned}
I\left(p_{b}(r, n)\right) & =-P_{b}^{\mathrm{top}}(r, n) \mathrm{e}_{\left(-1,0,[C]+r\left[C_{0}\right], n\right)}, \\
I(t(r, n)) & =-N^{\mathrm{top}}(r, n) \mathrm{e}_{\left(0,0, r\left[C_{0}\right], n\right)} .
\end{aligned}
$$

Employing the formalism reviewed above, Lemma A.4 translates into the following Hall algebra identities:

$$
\begin{gathered}
p_{b_{c}}(r, n)-p_{b_{+}}(r, n)=\sum_{\substack{r_{1}, r_{2}, n_{1}, n_{2} \in \mathbb{Z}, r_{1} \geq 1, r_{2} \geq 0 \\
r_{1}+r_{2}=r, n_{1}+n_{2}=n \\
n_{1} / r_{1}=-2 b_{c}}} s\left(r_{1}, n_{1}\right) * p_{b_{+}}\left(r_{2}, n_{2}\right) \\
p_{b_{c}}(r, n)-p_{b_{-}}(r, n)=\sum_{\substack{r_{1}, r_{2}, n_{1}, n_{2} \in \mathbb{Z}, r_{1} \geq 0, r_{2} \geq 1 \\
r_{1}+r_{2}=r, n_{1}+n_{2}=n \\
n_{2} / r_{2}=-2 b_{c}}} p_{b_{-}}\left(r_{1}, n_{1}\right) * s\left(r_{2}, n_{2}\right),
\end{gathered}
$$

for $b_{-}<b_{c}<b_{+}$sufficiently close to $b_{c}$. The sum in the right hand side is finite for fixed $(r, n)$ since there is a finite set of decompositions $r=$ $r_{1}+\cdots+r_{l}$ with $r_{i} \geq 1,1 \leq i \leq l$. Then repeating the steps in [8, Lemmas 2.1-2.4 ], identities (A.6) imply

$$
\begin{gathered}
p_{b_{-}}(r, n)-p_{b_{+}}(r, n)=\sum_{l \geq 2} \frac{(-1)^{l-1}}{(l-1) !} \sum_{\begin{array}{c}
\left(r_{i}, n_{i}\right) \in \mathbb{Z}^{2}, 1 \leq i \leq l, \\
r_{i} \geq 1,1 \leq i \leq l-1, r_{l} \geq 0 \\
r_{1}+\cdots+r_{l}=r, n_{1}+\cdots+n_{l}=n, \\
n_{i} / r_{i}=-2 b_{c}, 1 \leq i \leq l-1
\end{array}} \\
\quad \times\left[t\left(r_{1}, n_{1}\right), \ldots,\left[t\left(r_{l-1}, n_{l-1}\right), p_{b_{+}}\left(r_{l}, n_{l}\right)\right] \cdots\right] .
\end{gathered}
$$

Again, the sum in the right-hand side of Equation (A.7) is finite because the set of decompositions $r=r_{1}+\cdots+r_{l}$ with $r_{i} \geq 1,1 \leq i \leq l$ is finite. 
This identity is in fact a wall-crossing formula for stack functions. Applying the integration map to both sides of Equation (A.7) yields the following wall-crossing formula for topological Euler character invariants

$$
\begin{gathered}
P_{b_{-}}^{\text {top }}(r, n)-P_{b_{+}}^{\text {top }}(r, n) \\
=\sum_{l \geq 2} \frac{1}{(l-1) !} \sum_{\substack{\left(r_{i}, n_{i}\right) \in \mathbb{Z}^{2}, 1 \leq i \leq l, r_{i} \geq 1,1 \leq i \leq l-1, r_{l} \geq 0 \\
r_{1}+\cdots+r_{l}=r, n_{1}+\cdots+n_{l}=n, n_{i} / r_{i}=-2 b_{c}, 1 \leq i \leq l-1}}^{l-1} P_{b_{+}}^{\mathrm{top}}\left(r_{l}, n_{l}\right) \prod_{i=1}^{l} n_{i} N^{\mathrm{top}}\left(r_{i}, n_{i}\right) .
\end{gathered}
$$

\section{A.3. Summing over critical values}

Recall that $b>0$ is called a small stability parameter of type $(r, n)$ if there are no critical parameters of type $(r, n)$ in the interval $[0, b)$. All moduli stacks $\mathcal{P}_{(\omega, b)}(X, C, r, n)$ of $\mu_{(\omega, b)}$-semistable objects of $\mathcal{A}^{C}$ with numerical invariants $\left(-1,0,[C]+r\left[C_{0}\right], n\right)$ for small $b$ are canonically isomorphic, and will be denoted by $\mathcal{P}_{0+}(X, C, r, n)$. Combining the results of Proposition 3.6, Lemma 3.7, and Proposition 3.11, it follows that for any $(r, n) \in \mathbb{Z}_{\geq 0} \times \mathbb{Z}$, $\mathcal{P}_{0+}(X, C, r, n)$ is geometrically bijective to an $\mathbb{C}^{\times}$-gerbe over the relative Quot scheme $Q^{[l, r]}(C)$, where $l=n-\chi\left(\mathcal{O}_{C}\right)$. Then the topological Euler character invariants in this chamber are simply given by

$$
P_{0+}^{\text {top }}(r, n)=\chi\left(Q^{[l, r]}(C)\right) .
$$

If $n<\chi\left(\mathcal{O}_{C}\right)$ the stack $\mathcal{P}_{0+}(X, C, r, n)$ is empty, and $P_{0+}^{\text {top }}(r, n)=0$. Therefore in this chamber the generating function of topological invariants is

$$
Z^{\text {top }}(X, C, T, u)=u^{\chi\left(\mathcal{O}_{C}\right)} \sum_{l \geq 0} \sum_{r \geq 0} T^{r} u^{l} \chi\left(Q^{[l, r]}(C)\right) \text {. }
$$

At the same time, by analogy with [50, Thm. 3.21], there exists a constant $\mu_{r, n} \in \mathbb{R}$ depending only on $(r, n)$ such that for $b<-\mu_{r, n} / 2$, the moduli stack of $(\omega, b)$-semistable $C$-framed perverse coherent sheaves $\mathcal{P}_{(\omega, b)}(X, C, r, n)$ is isomorphic to the moduli stack of $C$-framed stable pairs. Hence for $b<$ $-\mu_{r, n} / 2$, the invariants $P_{b}(r, n)$ are equal to the topological Euler character invariants of stable pairs, denoted by $P_{-\infty}^{\text {top }}(r, n)$. Recall that Equation (1.14) is a factorization formula of the form

$$
Z_{-\infty}^{\mathrm{top}}(X, C, T, u)=Z^{\mathrm{top}}\left(X, C_{0}, T, u\right) Z_{0+}^{\mathrm{top}}(X, C, T, u)
$$


where

$$
Z_{-\infty}^{\mathrm{top}}(X, C, T, u)=\sum_{r \geq 0} \sum_{n \in \mathbb{Z}} T^{r} u^{n} P_{-\infty}^{\mathrm{top}}(r, n)
$$

and

$$
Z^{\text {top }}\left(X, C_{0}, T, u\right)=\sum_{r \geq 0} \sum_{n \in \mathbb{Z}} T^{r} u^{n} N^{\text {top }}(r, n) .
$$

This formula will be proven by successive applications of the wall-crossing formula (A.8).

First note that the set of critical parameters $-\mu_{r, n} / 2 \leq b_{c}<1 /(2 r)$ of type $(r, n)$ is finite since all such parameters must be of the form $b_{c}=-\frac{1}{2 r^{\prime}}$ with $1 \leq r^{\prime} \leq r$. For any $(r, n) \in \mathbb{Z}_{\geq 0} \times \mathbb{Z}$, let $P_{0-}^{\text {top }}(r, n)$ denote the value of $P_{b}^{\text {top }}(r, n)$ for any $b<0$ such that there are no critical parameters of type $(r, n)$ in the interval $[b, 0)$. Then note that the wall-crossing formula (A.8) at $b_{c}=0$ yields $P_{0+}^{\text {top }}(r, n)=P_{0-}^{\text {top }}(r, n)$. Therefore it suffices to relate $P_{-\infty}^{\text {top }}(r, n)$ to $P_{0-}^{\text {top }}(r, n)$.

Let $b_{-\infty}<\min \left\{0,-\mu_{r, n} / 2\right\}$ be an arbitrary stability parameter. $\Delta$ $\left(r, n ; b_{-\infty}\right)$ be the set of all decompositions

$$
r=r^{\prime}+\sum_{i=1}^{l} \sum_{j=1}^{k_{i}} r_{i, j}, \quad n=n^{\prime}+\sum_{i=1}^{l} \sum_{j=1}^{k_{i}} n_{i, j}
$$

with $l \geq 1, k_{i} \geq 1$ for all $1 \leq i \leq l, r^{\prime} \geq 0, r_{i, j} \geq 1$, satisfying

$$
\begin{aligned}
-b_{-\infty}>\frac{n_{1,1}}{r_{1,1}} & =\cdots=\frac{n_{1, k_{i}}}{r_{i, k_{i}}}>\frac{n_{2,1}}{r_{2,1}}=\cdots=\frac{n_{2, k_{i}}}{r_{2, k_{i}}}>\cdots>\frac{n_{l, 1}}{r_{l, 1}} \\
& =\cdots=\frac{n_{l, k_{l}}}{r_{l, k_{l}}}>0 .
\end{aligned}
$$

Note that this is a finite set for fixed $(r, n)$ and $b_{\infty}$. Then successive applications of Equation (A.8) yield

$$
\begin{aligned}
P_{-\infty}^{\mathrm{top}}(r, n)-P_{0-}^{\mathrm{top}}(r, n)= & \sum_{l \geq 1} \sum_{\left(r^{\prime}, r_{i, j}, n^{\prime}, n_{i, j}\right) \in \Delta\left(r, n ; b_{-\infty}\right)} P_{0-}^{\mathrm{top}}\left(r^{\prime}, n^{\prime}\right) \\
& \times \prod_{i=1}^{l} \prod_{j=1}^{k_{i}} \frac{1}{k_{i} !} n_{i, j} N^{\mathrm{top}}\left(r_{i, j}, n_{i, j}\right) .
\end{aligned}
$$


By simple combinatorics, the above equation may be rewritten as

$$
\begin{gathered}
P_{-\infty}^{\text {top }}(r, n)-P_{0-}^{\text {top }}(r, n) \\
=\sum_{l \geq 1} \frac{1}{l !} \sum_{\substack{\left(r^{\prime}, n^{\prime}\right),\left(r_{i}, n_{i}\right) \in \mathbb{Z}^{2}, 1 \leq i \leq l \\
r^{\prime} \geq 0, r_{i} \geq 1,1 \leq i \leq l, r^{\prime}+r_{1}+\cdots+r_{l}=r, n^{\prime}+n_{1}+\cdots+n_{l}=n, 0<n_{i} / r_{i}<-b_{-\infty}, 1 \leq i \leq l}}^{l} P_{0-}^{\mathrm{top}}\left(r^{\prime}, n^{\prime}\right) \prod_{i=1}^{l} n_{i} N^{\mathrm{top}}\left(r_{i}, n_{i}\right) .
\end{gathered}
$$

This formula holds for any $b_{-\infty}<\min \left\{0,-\mu_{r, n} / 2\right\}$. Moreover, the invariants $P_{0-}^{\text {top }}\left(r^{\prime}, n^{\prime}\right)$ are zero if $n^{\prime}<\chi\left(\mathcal{O}_{C}\right)$. Therefore, for $\left|b_{-\infty}\right|$ sufficiently large, the upper bound $n_{i} / r_{i}<-b_{-\infty}$ will be automatically satisfied. Hence, Equation (A.11) becomes

$$
\begin{gathered}
P_{-\infty}^{\mathrm{top}}(r, n)-P_{0-}^{\mathrm{top}}(r, n) \\
=\sum_{l \geq 1} \frac{1}{l !} \sum_{\substack{\left(r^{\prime}, n^{\prime}\right),\left(r_{i}, n_{i}\right) \in \mathbb{Z}^{2}, 1 \leq i \leq l \\
r^{\prime} \geq 0, r_{i} \geq 1,1 \leq i \leq l, r^{\prime}+r_{1}+\cdots+r_{l}=r, n^{\prime}+n_{1}+\cdots+n_{l}=n, n_{i} / r_{i}>0,1 \leq i \leq l}}^{l} P_{0-}^{\mathrm{top}}\left(r^{\prime}, n^{\prime}\right) \prod_{i=1}^{l} n_{i} N^{\mathrm{top}}\left(r_{i}, n_{i}\right), \\
\end{gathered}
$$

where the sum in the right-hand side is finite.

The last step is to convert Equation (A.12) into a relation between the generating functions. Multiplying (A.12) by $T^{r} u^{n}$ and summing over $r \geq 0$, $n \geq 1$ yields

$$
Z_{-\infty}^{\mathrm{top}}(X, C, T, u)=\exp \left[\sum_{r>0} \sum_{n>0}(-1)^{n} n N^{\mathrm{top}}(r, n) T^{r} u^{n}\right] Z_{0-}^{\mathrm{top}}(X, C, T, u)
$$

Now Lemma 2.4 implies that the moduli stack of $\omega$-slope semistable sheaves $F$ with topological support on $C_{0}$ and $\operatorname{ch}_{2}(F)=r\left[C_{0}\right], \chi(F)=n$ is isomorphic to the moduli stack of semistable rank $r$ bundles $E$ on $\mathbb{P}^{1}$ with $\chi(E)=n$. If $n$ is not a multiple of $r$ there are no such bundles. If $n=k r, k \in \mathbb{Z}$, there is only one such bundle up to isomorphism, $\mathcal{O}_{\mathbb{P}^{1}}(k-1)^{\oplus r}$. Therefore the moduli stack is empty unless $n=k r, k \in \mathbb{Z}$, in which case it is isomorphic 
to the quotient stack $[\operatorname{Spec}(\mathbb{C}) / G L(r, \mathbb{C})]$. Then $[24$, Ex. 6.2] shows that

$$
N^{\text {top }}(r, n)= \begin{cases}\frac{(-1)^{r-1}}{r^{2}}, & \text { if } n \equiv 0 \bmod r \\ 0, & \text { otherwise }\end{cases}
$$

By direct substitution,

$$
\begin{aligned}
\sum_{r>0} \sum_{n>0} n N^{\mathrm{top}}(r, n) T^{r} u^{n} & =\sum_{k \geq 1} k \sum_{r \geq 1} \frac{(-1)^{r-1}}{r}\left(T u^{k}\right)^{r} \\
& \left.=\sum_{k \geq 1} \ln \left(1+T u^{k}\right)\right) \\
& \left.=\ln \prod_{k \geq 1}\left(1+T u^{k}\right)\right)^{k}
\end{aligned}
$$

Hence,

$$
\left.\exp \left[\sum_{r>0} \sum_{n>0}(-1)^{n} n N^{\mathrm{top}}(r, n) T^{r} u^{n}\right]=\prod_{k \geq 1}\left(1+T u^{k}\right)\right)^{k}
$$

In order to conclude the proof, it remains to show that

$$
\left.Z_{0}^{\mathrm{top}}(X, T, u)=\prod_{k \geq 1}\left(1+T u^{k}\right)\right)^{k}
$$

Since the formal neighborhood of $C_{0}$ in $X$ is isomorphic to the formal neighborhood of the zero section in the total space $Y$ of $\mathcal{O}_{\mathbb{P}^{1}}(-1)^{\oplus 2}$, it suffices to prove the corresponding result for stable pairs on $Y$. This follows from [40, Thm. 3.15], which proves analogous formulas for counting invariants defined by integration with respect to Behrend's constructible function. For concreteness note that the variables $q_{0}, q_{1}$ used in [40, Thm. 3.15] are related to $T, u$ by

$$
q_{1}^{-1}=T, \quad q_{0} q_{1}=u .
$$

Moreover, Equation (3.4) in [40, Thm. 3.15] and the last formula in [40, Section 3.2] yield

$$
\left.Z^{\mathrm{top}}(Y, T, u)=\prod_{k \geq 1}\left(1+T u^{k}\right)\right)^{k}
$$

as claimed above. 


\section{References}

[1] A. Bayer, Polynomial Bridgeland stability conditions and the large volume limit, Geom. Topol. 13(4) (2009), 2389-2425.

[2] K. Behrend, Donaldson-Thomas type invariants via microlocal geometry, Ann. Math. (2) 170(3) (2009), 1307-1338.

[3] K. Behrend, J. Bryan and B. Szendroi, Motivic degree zero DonaldsonThomas invariants, arXiv.org:0909.5088.

[4] M. Bender and S. Mozgovoy, Crepant resolutions and brane tilings II: tilting bundles, arXiv:0909.2013.

[5] J. Cheah, On the cohomology of the Hilbert scheme of points, J. Alg. Geom. 5 (1996), 479-511.

[6] W.-Y. Chuang, D.-E. Diaconescu and G. Pan, Rank two ADHM invariants and wallcrossing, Commun. Numer. Theor. Phys. 4 (2010), 417461. arXiv: 1002.0579.

[7] W.-Y. Chuang, D.-E. Diaconescu and G. Pan, Wallcrossing and cohomology of the moduli space of Hitchin pairs, Commun. Numer. Theor. Phys. 5 (2011), 1-56.

[8] W.-Y. Chuang, D.-E. Diaconescu and G. Pan, Chamber structure and wallcrossing in the ADHM theory of curves II, J. Geom. Phys. 62(2) (2012), 548-561.

[9] B. Davison, Invariance of orientation data for ind-constructible CalabiYau $A_{\infty}$ categories under derived equivalence, arXiv:1006.5475.

[10] D.-E. Diaconescu and B. Florea, Large $N$ duality for compact CalabiYau threefolds, Adv. Theor. Math. Phys. 9(1) (2005), 31-128.

[11] D.-E. Diaconescu, V. Shende and C. Vafa, Large $N$ duality, Lagrangian cycles and algebraic knots, arXiv:1111.6533.

[12] T. Dimofte and S. Gukov, Refined, motivic, and quantum, Lett. Math. Phys. 91 (2010), 1.

[13] N.M. Dunfield, S. Gukov and J. Rasmussen, The superpolynomial for knot homologies, Exp. Math. 15(2) (2006), 129-159.

[14] R. Gopakumar and C. Vafa, $M$ theory and topological strings. 2. arXiv:hep-th/9812127. 
598 Duiliu-Emanuel Diaconescu, Zheng Hua and Yan Soibelman

[15] R. Gopakumar and C. Vafa, M-theory and topological strings. I, arXiv:hep-th/9809187.

[16] S. Gukov, A.S. Schwarz and C. Vafa, Khovanov-Rozansky homology and topological strings, Lett. Math. Phys. 74 (2005), 53-74.

[17] Z. Hua, Chern-Simons functions on toric Calabi-Yau threefolds and Donaldson-Thomas theory, arXiv:1103.1921.

[18] D. Huybrechts and M. Lehn, Framed modules and their moduli, Int. J. Math. 6(2) (1995), 297-324.

[19] D. Huybrechts and M. Lehn, The geometry of moduli spaces of sheaves, Cambridge Mathematical Library. Cambridge University Press, Cambridge, 2nd edn, 2010.

[20] D. Joyce, Configurations in abelian categories. I. Basic properties and moduli stacks, Adv. Math. 203(1) (2006), 194-255.

[21] D. Joyce, Configurations in abelian categories. II. Ringel-Hall algebras, Adv. Math. 210(2) (2007), 635-706.

[22] D. Joyce, Configurations in abelian categories. III. Stability conditions and identities, Adv. Math. 215(1) (2007), 153-219.

[23] D. Joyce, Configurations in abelian categories. IV. Invariants and changing stability conditions, Adv. Math. 217(1) (2008), 125-204.

[24] D. Joyce and Y. Song, A theory of generalized Donaldson-Thomas invariants, arXiv.org:0810.5645.

[25] S. Katz and C.-C. M. Liu, Enumerative geometry of stable maps with Lagrangian boundary conditions and multiple covers of the disc, Adv. Theor. Math. Phys. 5(1) (2001), 1-49.

[26] M. Kontsevich and Y. Soibelman, Stability structures, Donaldson-Thomas invariants and cluster transformations, arXiv .org:0811.2435.

[27] M. Kontsevich and Y. Soibelman, Cohomological Hall algebra, exponential Hodge structures and motivic Donaldson-Thomas invariants, Commun. Num. Theor. Phys. 5 (2011) 231-352.

[28] J.M.F. Labastida, M. Marino and C. Vafa, Knots, links and branes at large N. J. High Energy Phys. 11 (2000) 007. 
[29] C.-C.M. Liu, K. Liu and J. Zhou, A proof of a conjecture of MariñoVafa on Hodge integrals, J. Differ. Geom. 65(2) (2003), 289-340.

[30] C.-C.M. Liu, K. Liu and J. Zhou, A formula of two-partition Hodge integrals J. Amer. Math. Soc. 20(1) (2007), 149-184 (electronic).

[31] M. Marino and C. Vafa, Framed knots at large $N$, arXiv:hep-th/0108064.

[32] D. Maulik, N. Nekrasov, A. Okounkov and R. Pandharipande, GromovWitten theory and Donaldson-Thomas theory. I, Composit. Math. 142(5) (2006), 1263-1285.

[33] D. Maulik, A. Oblomkov, A. Okounkov and R. Pandharipande, Gromov-Witten/Donaldson-Thomas correspondence for toric 3-folds, arXiv:0809. 3976.

[34] D. Maulik, R. Pandharipande and R. P. Thomas, Curves on K3 surfaces and modular forms, J. Topol. 3(4) (2010), 937-996; with an appendix by A. Pixton.

[35] D. Maulik and Z. Yun, Macdonald formula for curves with planar singularities, arXiv:1107.2175.

[36] L. Migliorini and V. Shende, A support theorem for Hilbert schemes of planar curves, arXiv:1107.2355.

[37] A. Morrison, S. Mozgovoy, K. Nagao and B. Szendroi, Motivic Donaldson-Thomas invariants of the conifold and the refined topological vertex, arXiv:1107.5017.

[38] A. Morrison and K. Nagao, Motivic Donaldson-Thomas invariants of toric small crepant resolutions, arXiv:1110.5976.

[39] D.R. Morrison and C. Vafa, Compactifications of F theory on CalabiYau threefolds. 2., Nucl. Phys. B 476 (1996), 437-469.

[40] K. Nagao and H. Nakajima, Counting invariant of perverse coherent sheaves and its wall-crossing, Int. Math. Res. Not. 17 (2011), 3885-3938.

[41] A. Oblomkov, J. Rasmussen and V. Shende, The Hilbert scheme of a plane curve singularity and the HOMFLY homology of its link, arXiv: 1201.2115. 
600 Duiliu-Emanuel Diaconescu, Zheng Hua and Yan Soibelman

[42] A. Oblomkov and V. Shende, The Hilbert scheme of a plane curve singularity and the HOMFLY polynomial of its link, arXiv:1003.1568.

[43] A. Okounkov and R. Pandharipande, Hodge integrals and invariants of the unknot, Geom. Topol. 8 (2004), 675-699.

[44] H. Ooguri and C. Vafa, Knot invariants and topological strings, Nucl. Phys. B 577 (2000), 419-438.

[45] R. Pandharipande and R.P. Thomas, Curve counting via stable pairs in the derived category, Invent. Math. 178(2) (2009), 407-447.

[46] R. Pandharipande and R.P. Thomas, Stable pairs and BPS invariants, J. Am. Math. Soc. 23(1) (2010), 267-297.

[47] V. Shende, Hilbert schemes of points on a locally planar curve and the Severi strata of its versal deformation, arXiv:1009.0914.

[48] B. Szendröi, Non-commutative Donaldson-Thomas invariants and the conifold, Geom. Topol. 12(2) (2008), 1171-1202.

[49] L.Q. Thuong, Proofs of the integral identity conjecture over algebraically closed fields, arXiv:1206.5334.

[50] Y. Toda, Generating functions of stable pair invariants via wallcrossings in derived categories, arXiv.org:0806.0062.

[51] Y. Toda, Limit stable objects on Calabi-Yau 3-folds, arXiv.org:0803.2356.

NHETC

Rutgers University

PiscataWAY

NJ 08854-0849, USA

E-mail address: duiliu@physics.rutgers.edu

Department of Mathematics

Kansas State University

MANHATTAN

KS 66506-2602, USA

E-mail address: zheng.hua.zju@gmail.com

Department of Mathematics

Kansas State University

MANHATTAN

KS 66506-2602, USA

E-mail address: soibel@math.ksu.edu

ReCeIVed April 9, 2012 